UCRL-LR-114125

Distribution Category UC-600

\title{
Optimal Groundwater Remediation Using Artificial \\ Neural Networks and the Genetic Algorithm
}

\author{
Leah L. Rogers \\ (Ph.D. Thesis)
}

Manuscript date: August 1992

LAWRENCE LIVERMORE NATIONAL LABORATORY University of California • Livermore, California • 94551

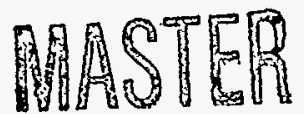




\section{DISCLAIMER}

This report was prepared as an account of work sponsored by an agency of the United States Government. Neither the United States Government nor any agency thereof, nor any of their employees, make any warranty, express or implied, or assumes any legal liability or responsibility for the accuracy, completeness, or usefulness of any information, apparatus, product, or process disclosed, or represents that its use would not infringe privately owned rights. Reference herein to any specific commercial product, process, or service by trade name, trademark, manufacturer, or otherwise does not necessarily constitute or imply its endorsement, recommendation, or favoring by the United States Government or any agency thereof. The views and opinions of authors expressed herein do not necessarily state or reflect those of the United States Government or any agency thereof. 


\section{DISCLAIMER}

Portions of this document may be illegible in electronic image products. Images are produced from the best available original document. 
OPTIMAL GROUNDWATER REMEDIATION USING

ARTIFICIAL NEURAL NETWORKS AND THE GENETIC ALGORITHM

\section{A DISSERTATION}

SUBMITTED TO THE DEPARTMENT OF APPLIED EARTH SCIENCES AND THE COMMITTEE ON GRADUATE STUDIES OF STANFORD UNIVERSITY

IN PARTIAL FULFILLMENT OF THE REQUIREMENTS

FOR THE DEGREE OF

DOCTOR OF PHILOSOPHY

Leah Lucille Rogers

August 1992 
I certify that I have read this thesis and that in my opinion it is fully adequate, in scope and quality, as a dissertation for the degree of Doctor of Philosophy.

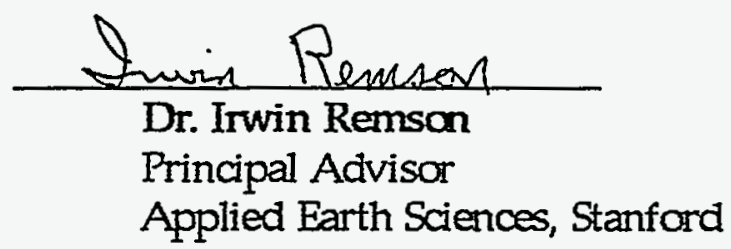

I certify that I have read this thesis and that in my opinion it is fully adequate, in scope and quality, as a dissertation for the degree of Doctor of Philosophy.

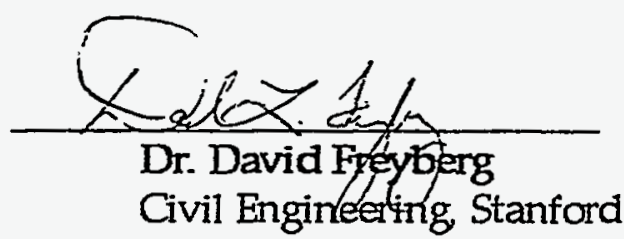

I certify that I have read this thesis and that in my opinion it is fully adequate, in scope and quality, as a dissertation for the degree of Doctor of Philosophy.

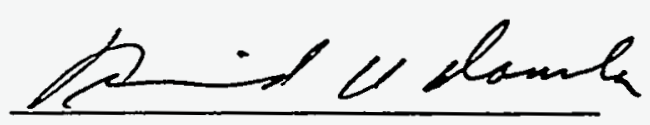

Dr. Farid Dowla

Earth Sciences, Lawrence

Livermore National Laboratory

Approved for the University Committee on Graduate Studies: 
$-\ldots,-\infty-\infty-\infty-\cdots$ 


\section{Acknowledgements}

In looking back over the twists and turns in this research, I have many individuals to thank. My academic advisor, Irwin Remson, was always understanding and insightful in his vision of the multiple paths one can take in dissertation research. John Bredehoeft was clearthinking and determined, and supported my ideas for new research when many others were filled with doubt. I am deeply grateful to Irwin and John for giving me a long enough rope to eventually ease over the cliff of artificial neural networks and pulling me back up so I could tell the wondrous tale of what I had seen. I would like to thank my dissertation committee for their careful review of my work and countless helpful discussions on research strategies. Along with Irwin and John, the committee members who helped forge this research were David Freyberg, Andre Journel, Peter Kitanidis, and Paul Roberts.

My neural networks gurus were Farid Dowla and David Rumelhart. They shared with me their invaluable knowledge and insight. I have benefited greatly from their interactiveness and helpful natures. Also thanks to Erik Johansson and Sean Lehman who gave permission to use their backpropagation code and Gyu-sang Jang who shared his knowledge of the weightelimination implementation.

I have been most fortunate in having many supportive project leaders, managers, and colleagues at LLNL. They supported me over the years in this professional development of coursework and dissertation research, looking to the long-term benefits instead of the immediate inconvenience of not having me there full time. Many, many thanks to Fred Hoffman, Bill Isherwood, Bill McConachie, Larry McKague, and Lee Younker. Special thanks to Richard Knapp for his nurturing leadership of our flow and transport cadre and to John Ziagos under whose banner we are creating a renaissance of optimization. Thanks to Andy Tompson, John Nitao, Tom Busheck, Robin Newmark, Eric Nichols, Nai-Hsien Mao, Ed Kansa, Robin Reichlin, 
Jerry Sweeney and Jay Zucca for moral support as well as long discussions on hydrology and the meaning of life. Thanks also to David Rice, Jr. and Dorothy Bishop, co-principal investagators, who had patience with me and visionary ideas of remediation and characterization. Virginia Johnson was an invaluable statistical font of knowledge and a fellow zealot of neural networks. Bob Herrick, Betsy Foote, and Cheryl Ham helped me immeasurably with programming and graphics hurdles.

Over the years many fellow Stanford students helped me to maintain a healthy perspective on the process. I am especially grateful to Jean Bahr, Hedeff Essaid, Eric Reichard, Steve Ingibritsen, Stuart Rojstaczer, Chris Kolterman, and Robert Gailey. And a very special thanks to the late Tom Black who was always an enlightened and supportive friend.

Finally I come to thank my family. It was their core of love and good nature that gave me the greatest strength. My gratitude is endless to my parents, Robert and Kuhne Rogers and to my siblings Mark and Libby. Their determination and love of learning was a constant inspiration. There also should be an appendix of thanks to my supportive entended family of loving grandmother Lucile, aunts, uncles, cousins, and loved-ones-in-law. And it is with great love that I thank my long-suffering husband Gregory Faris. I have been in graduate school the entire time he has known me, through courtship, marriage, and the birth of our now two-year old Anna Elizabeth. He has never lost faith and has always been an enlightened and understanding companion. 


\section{Table of Contents}

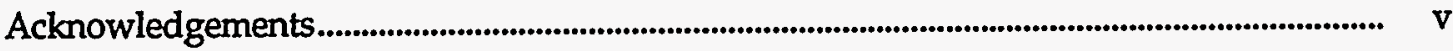

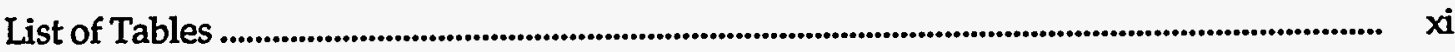

List of Figures................................................................................................................................ xiii

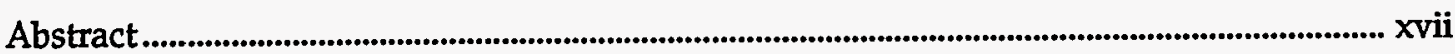

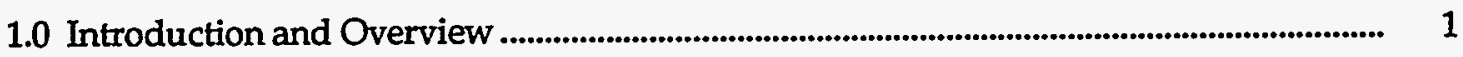

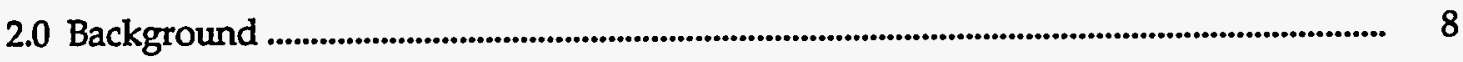

2.1 Groundwater Management ........................................................................................ 8

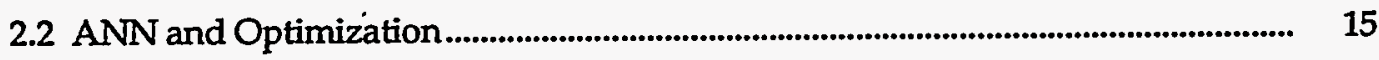

2.2.1 Early Work............................................................................................................. 15

2.2.2 Optimization.......................................................................................................... 16

2.2.3 Rapid Growth...................................................................................................... 17

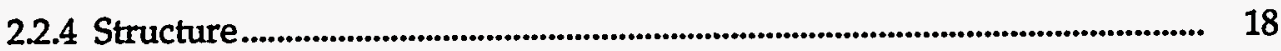

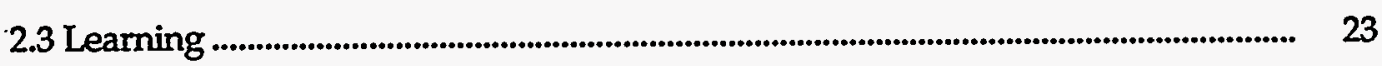

2.3.1 Hebbian Leaming ................................................................................................ 24

2.3.2 Widrow-Hoff Learning ................................................................................... 24

2.3.3 Perceptron Learning .......................................................................................... 25

2.3.4 Backpropagation Learning ............................................................................... 25

2.3.5 Conjugate Gradient Method ............................................................................ 29

2.3.6 Weight Elimination .......................................................................................... 31

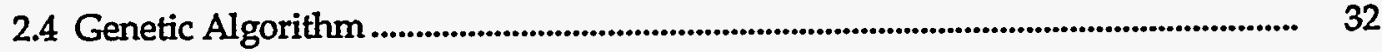

3.0 Groundwater Management Modeling using ANNs............................................................ 41

3.1 Implementation of ANN Groundwater Management Model ................................... 41

3.1.1 Illustrative Example: Three-well Remediation Problem.............................. 42 
3.1.2 Solute Transport Modeling ......................................................................... 46

3.2 The Twenty-well Remediation Problem .............................................................................. 48

3.2.1 Conceptualization and Formulation ............................................................ 48

3.2.2 Incorporation of the Management Formulation into the ANN

Architecture

3.2.3 Groundwater Flow and Transport Simulations (in Parallel) to

Develop Training and Testing Patterns ........................................................... 52

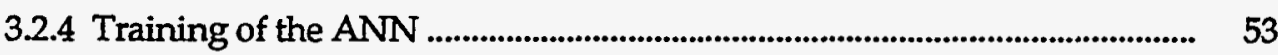

3.2.4.1 Measure of Success or Performance.................................................. 54

3.2.4.2 Training Rounds........................................................................... 54

3.2.4.3 Weight Elimination to Improve Network Generalization

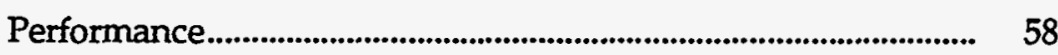

3.2.5 Success Prediction of Combinatorial Management Possibilities ................ 59

3.2.6 Confirmation of Management Solutions ................................................... 62

3.2.7 Comparison with a More Conventional Technique ..................................... 62

3.2.8 Multiple Objectives ................................................................................. 67

3.2.9 Discussion of Twenty-well Remediation Problem ...................................... 68

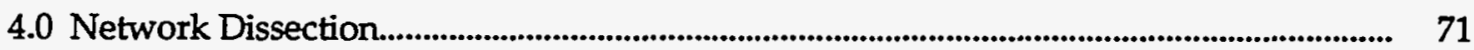

4.1 Simple Example of Weight Maturation .................................................................. 72

4.2 Statistical and Spatial Description of Weights ............................................................ 76

4.3 Well Sensitivity Analysis .................................................................................. 77

4.4 Network Complexity ........................................................................................................ 91

5.0 Field-Scale Applications......................................................................................................... 94

5.1 Predicting Mass Output .................................................................................... 94

5.2 Multiple Objectives ............................................................................................. 112

5.2.1 Network Training ..................................................................................... 114

5.2.2 Optimization ........................................................................................................ 114 
6.0 Issues and Analogies

6.1 Appropriate Number of Training Patterns................................................................... 123

6.2 Appropirate Number of Hidden Layer Nodes .......................................................... 125

6.3 Improvement of Learning Rates............................................................................... 125

6.4 Generalization Abilities ............................................................................................... 126

7.0 Summary and Future Directions of ANN Groundwater Management ............................. 129

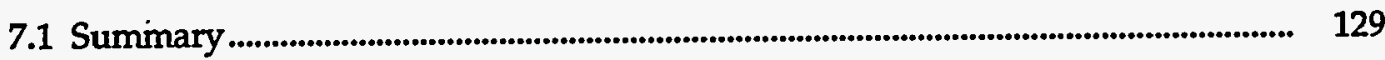

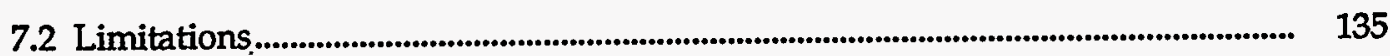

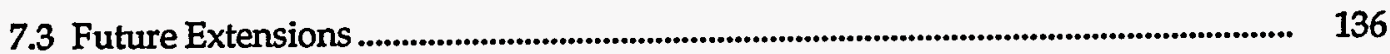

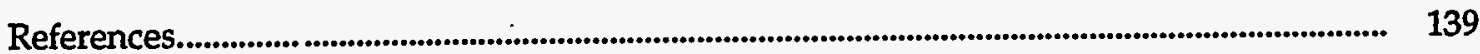

Appendix A: Conjugate Gradient Backpropagation Learning Algorithm .............................. 148

Appendix B: Nonlinear Programming Method of Groundwater Management ...................... 152 


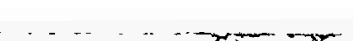

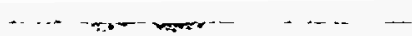

. $\Rightarrow x+$ 


\section{List of Tables}

Table 1. Transfer of information from one generation to the next according to the genetic algorithm for a population of 20 for the twenty-well remediation problem. a) population of current generation, $b$ ) mating pool after reproduction, c) example of crossover operator for first two matings, and d) population of new generation................................................................................................

Table 2. Ilustrative example. a) input and output patterns, and

b) ANN performance. 44

Table 3. Aquifer and transport parameter values and model specifications 53

Table 4. Accuracy and generalization performances for seven ANN training rounds.. 56

Table 5. Multiple objectives for the twenty-well test remediation. 68

Table 6. Univariate statistics for weight sets $w a, w b, w c$, and wd... 76

Table 7. Rankings of input vector (well) sensitivity for a) weight set wa,

b) weight set $w b, c)$ weight set $w c$, and d) weight set wd.

Table 8. Rankings of input vector (well) sensitivity for a) weight set $175 \mathrm{wa}$,

b) weight set $175 \mathrm{wb} 7, \mathrm{c}$ ) weight set $175 \mathrm{wa9}$, and d) weight set $175 \mathrm{wb} 9$. 107

Table 9. Transfer of information from one generation to the next according to the genetic algorithm for the multiple objectives field-scale application. a) a current generation, b) mating pool after reproduction, c) crossover operator, and d) a new generation. 
$\ldots+\ldots+\ldots$ 


\section{List of Figures}

Figure 1. Flowchart comparing nonlinear programming (NLP) and artificial neural network (ANN) groundwater management.

Figure 2. Neural networks: (a) Architecture of a biological prototype.

(b) Artificial digital prototype of a neuron.

Figure 3. Structure of a multilayered neural network with a five-unit input layer, a three-unit hidden layer, and a one-unit output layer. Note that there are 18 weights in this network.

Figure 4: Weight elimination penalty function (on $z$ axis) versus weight magnitudes (on the $y$ axis) and parameter wo (on the $x$ axis). 33

Figure 5. The genetic algorithm: analogies 35

Figure 6. The genetic algorithm: crossover operator. 36

Figure 7. Illustrative example of ANN groundwater remediation. (a) Numerical simulation grid with three possible pumping wells downgradient from a contaminant plume and upgradient from a line of monitor wells. (b) Mapping from conceptualization to a decision on remedial success. (c) ANN input and output patterns for all eight possible pumping realizations.

Figure 8. Twenty-well example of ANN groundwater remediation. (a) Possible locations of remediation wells. (b) Numerical mesh.

Figure 9. XYZ plot of training sytem error, versus number of iterations, versus testing system error in weight elimination training for input series (a) $1,2,3,5$, (b) $2,3,4,5$,

(c) $1,3,4,5$, and (d) $1,2,4,5$.

Figure 10. Seven recommended management solutions from the ANN groundwater management model [(a) through $(\mathrm{g})]$, compared with a recommended solution from a nonlinear programming (NPL) management model (h).

Figure 11. Unremediated concentration histories at the monitor wells (a), and unremediated aerial-plume concentration contours over time $[(b)$ through $(f)]$..

Figure 12. Successful ANN management solution A: Concentration histories at the monitor wells (a), and aerial-plume concentration contours over time [(b) through (f)]. 
Figure 13. Successful ANN management solution F: Concentration histories at the monitor wells (a), and aerial-plume concentration contours over time [(b) through (f)].

Figure 14. Network weights after training with a) one pattern, b) two patterns,

c) three patterns, and 4) four patterns.

Figure 15. Performance results and network weights for the architecture 21-7-1 in a three-dimensional historgram for infile 1 with weight set $w b$

Figure 16. Performance results and network weights for the architecture 21-7-1 in a three-dimensional histogram for infile 3 weight set $w c$.

Figure 17. Barchart of network weights for a single hidden node.

Figure 18. Percentage of altered outcomes by tweaked input vector for -5.0 perturbation

Figure 19. Percentage of altered outcomes by tweaked input vector for 5.0 perturbation

Figure 20. Percentage of altered outcomes by tweaked input vector for -10.0 perturbation.

Figure 21. Percentage of altered outcomes by tweaked input vector for 10.0 perturbation.

Figure 22. Performance results and network weights for the architecture 21-7-1 in a three-dimensional histogram for infile 3 with no weight elimination.

Figure 23. Performance results and network weights for the architecture 21-7-1 in a three-dimensional histogram for infile 3 with weight elimination.

Figure 24. LLNL Site. (a) Volatile organic compounds concentration contours for the LLNL site. Also shown are the 28 well locations with 23 pumping (yellow circles) and 5 injection (white circles) locations. (b) 28 well locations with 23 pumping (yellow circles) and 5 injection (white circles) locations.

Figure 25. Predicted versus actual mass removed from 50 test pumping patterns for network trained with set 175a and architecture of a) 29-5-1, b) 29-7-1, and c) 29-9-1. Mass in $\mathrm{Kg}$ is normalized.

Figure 26. Predicted versus actual mass removed from 50 test pumping patterns for network trained with set $175 \mathrm{~b}$ and architecture of a) 29-5-1, b) 29-7-1, and c) 29-9-1. Mass in $\mathrm{Kg}$ is normalized.

Figure 27. Residuals between predicted and actual mass removed from 50 test pumping patterns for network trained with set 175a and architecture of a) 29-5-1, b) 29-7-1, and c) 29-9-1. 
Figure 28. Residuals between predicted and actual mass removed from 50 test pumping patterns for network trained with set $175 \mathrm{~b}$ and architecture of a) 29-5-1, b) 29-7-1, and c) 29-9-1. 103

Figure 29. Mass output prediction: barchart of sum of absolute value of residuals for different hidden node architectures.

Figure 30. Mass output prediction: barchart of mean of absolute value of residuals for different hidden node architectures.

Figure 31. Well locations for the mass output prediction with highlighting of locations determined by well sensitivity analysis (with weight permutation) to be important.

Figure 32. Numerical grid and boundaries for SUTRA flow and transport simulation at LLNL

Figure 33. Predicted versus actual cost of remediation for the network trained to two outputs. Cost is normalized.

Figure 34. Predicted versus actual cost of remediation for the network trained to only one output. Cost is normalized.

Figure 35. Flowchart of three ANNs trained for three-objective remediation. 117

Figure 36. Pressure and concentration contours over 40 years for SUTRA simulation of unremediated plume.

Figure 37. Pressure and concentration contours over 40 years for SUTRA simulation of all 28 remediation wells on. Estimated cost of remediation is $\$ 155$ million..........

Figure 38. Pressure and concentration contours over 40 years for SUTRA simulation of 19 remediation wells on. Estimated cost of remediation is $\$ 119$ million.

Figure 39. Plot of network complexity versus number of training examples. 127

Figure 40. Design strengths of ANN groundwater management. 131 


\section{Abstract}

An innovative computational approach for the optimization of groundwater remediation is presented which uses artificial neural networks (ANNs) and the genetic algorithm (GA). In this approach, the ANN is trained to predict an aspect of the outcome of a flow and transport simulation. Then the GA searches through realizations or patterns of pumping and uses the trained network topredict the outcome of the realizations. This approach has advantages of parallel processing of the groundwater simulations and the ability to "recycle" or reuse the base of knowledge formed by these simulations. These advantages offer reduction of computational burden of the groundwater simulations relative to a more conventional approach which uses nonlinear programming (NLP) with a quasi-newtonian search. Also the modular nature of this approach facilitates substitution of different groundwater simulation models.

The ANN technology, inspired by neurobiological theories of massive interconnection and parallelism, has been applied to a variety of optimization problems. In the ANN groundwater management approach presented here, the behavior of complex groundwater scenarios with spatially-variable transport parameters and multiple contaminant plumes are simulated with 2-D flow and transport codes. An ANN is trained upon a set of examples developed from groundwater simulations. The input to the ANN characterizes the different realizations of pumping. The output characterizes the objectives and constraints of the optimization, such as whether regulatory goals have been met, value of cost functions or cleanup time, and mass of contaminant removal.

The supervised learning algorithm of backpropagation is used to train the network. The conjugate gradient method and weight-elimination procedures are used to speed convergence and improve performance, respectively. Then a search is made through possible pumping realizations to find optimal realizations. The search is directed by a simple genetic algorithm 
which was developed in analogy to natural selection and genetics. The GA generates new possible pumping realizations which are presented to the trained network, the network then predicts the success of remediation for each pumping realization.

In example remediations, the optimal pumping realizations from the ANN approach are consistent with those resulting from the more conventional NLP approach, which involves NLP with a quasi-newtonian search. These consistent ANN and NLP solutions resulted from approximately the same number of groundwater simulations. However, the NLP approach requires sequential groundwater simulations while the ANN approach allows for the simulations to be run all at the same time, i.e., in parallel. This is a significant computational simplification, as the groundwater simulations are often the primary computational component in the groundwater management models.

ANN performance was observed upon variation of the problem formulation, network architecture, and learning algorithm. Field-scale applications and exploration of network generalization abilities are also presented. 


\subsection{Introduction and Overview}

In this work the term groundwater management is used to refer to a formal methodology which is capable of optimizing decisions about groundwater. Many diverse applications have been seen in such areas as decisions on dewatering of mines, arbitration of conflicting water supply issues, and improvement or maintenance of water quality. In the latter example, groundwater management seeks to minimize the impact of saltwater intrusion, hazardous wastes, pesticide use, or other threats to groundwater quality. This dissertation focuses on remediation or clean-up of contaminated groundwater systems and how decisions are made on locations and pumping schedules of remediation wells in order to optimize various measures of the success of remediation. The optimization techniques used solve the nonlinear groundwater management problem of simultaneously considering groundwater hydraulics and solute mass transport. The problem is nonlinear because the transport of contaminants is dependent on the velocity of groundwater. The velocity field, however, is affected by the pumping rates determined during optimization. Thus, to consider the effects of remedial pumping on the aquifer's concentrations, one must consider a transport equation that is nonlinear with unknown velocities multiplied by unknown concentrations.

The success of a groundwater management solution to a particular remediation problem depends to a significant degree on the quality of the flow and transport simulations used to predict the movement of contaminants over time. The flow and transport simulations also comprise the major computational effort involved in the groundwater management methodology. The general trend is towards more complex flow and transport simulations as our understanding of the physics of transport becomes more detailed and our interest in the variation of transport parameters or certainty of predictions encourages us to approach the simulations stochastically. This research contributes to groundwater management methodology by introducing an 
innovative computational approach for optimization which involves use of artificial neural networks (ANNs) and the genetic algorithm (GA). In this approach, the ANN is trained to predict a particular aspect of the outcome of the flow and transport simulation. Then the GA directs a search through possible management solutions, in this case patterns or realizations of pumping. These pumping realizations are presented to the trained ANN which predicts the outcome of the pumping realizations. The primary advantages of the ANN approach are parallel processing for the flow and transport component and the ability to "recycle" or reuse the base of knowledge formed by these flow and transport runs (Figure 1). In this work, these advantages will be demonstrated to enable reduction of computational burden of the calls to the flow and transport code relative to a more conventional approach which uses nonlinear programming (NLP) with a quasi-newtonian search. Also there is considerable independence of the flow and transport simulation from the optimization, which makes it easier to substitute one flow and transport code for another. For example, because no arrays are passed back and forth between the simulations and the ANN during the search for optimal pumping realizations, a threedimensional, three-phase (gas, liquid, solid) model could easily be subsituted for the twodimensional, two-phase (liquid-solid) model used in this work.

The dichotomy between our conventional digital computers and new theories of human perception, cognition, language, and memory has motivated a new approach to computation and computer architecture. The connectionist movement, believing that intelligence manifests itself from the communication of large numbers of simple processing units [Rumelhart and McClelland, 1986] has generated a new emphasis on the advantages of parallel structures of computer architecture. One such computational system, an artificial neural network (ANN), crudely resembling the human brain, is well-suited to such tasks as pattern recognition, combinatorial optimization, and discrimination. The ANN learns to solve a problem by pursuing multiple hypotheses in parallel and developing a memory capable of associating a large number of input patterns with a resulting set of outputs or effects. 


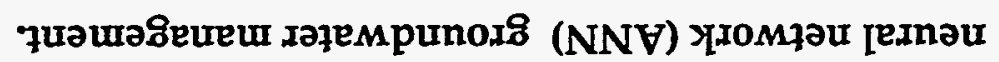

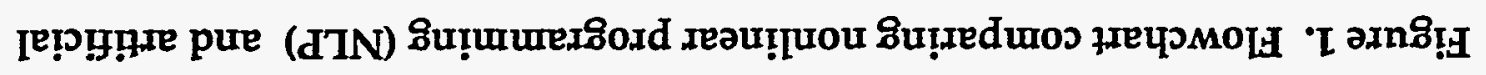
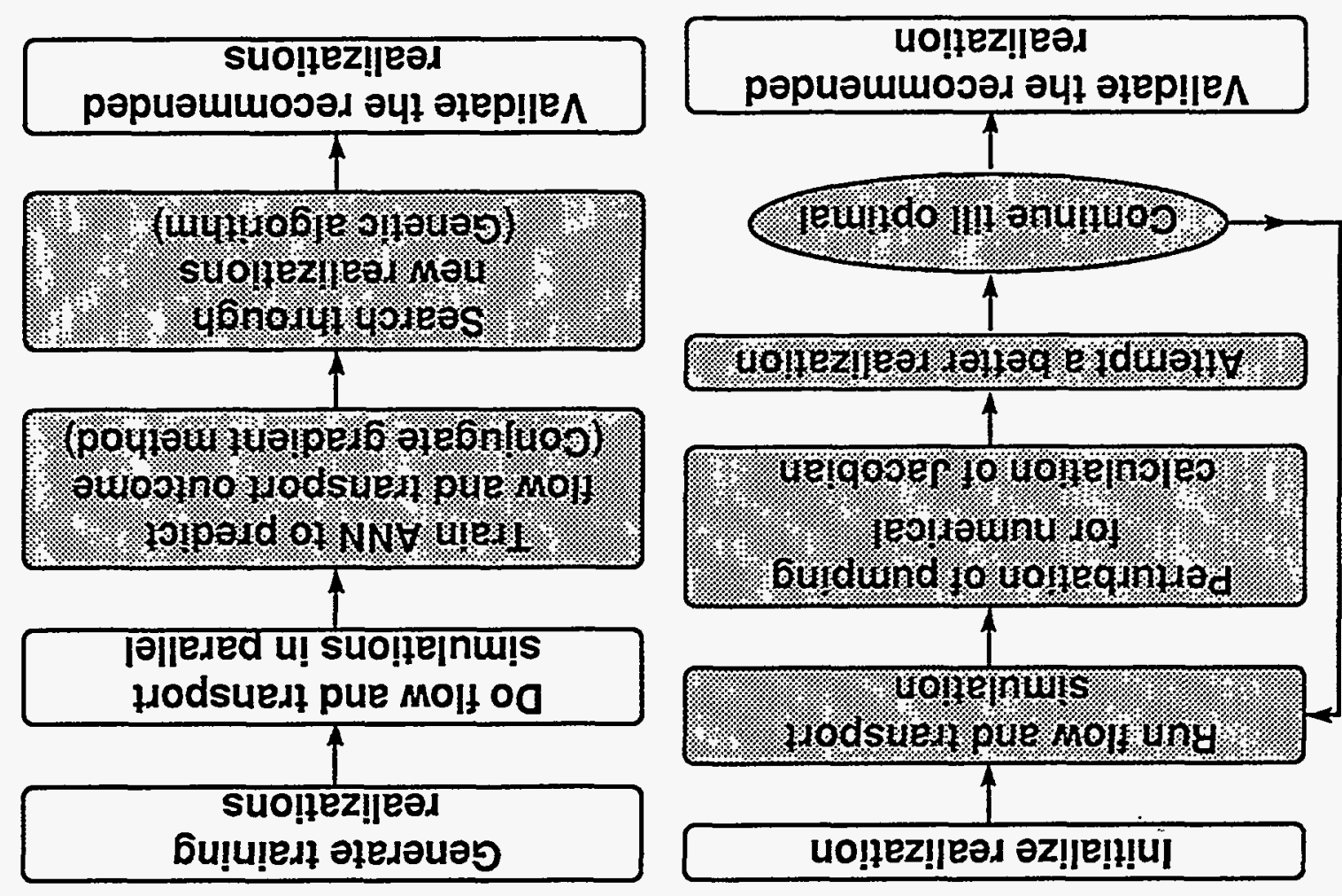

NNV 4t!M

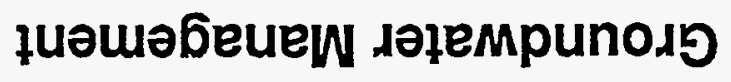

d TN $4 ? ! M$ quәuәбеนеW dәןемpuno. 
The ANN technology offers a promising new approach to analyzing a wide variety of complex patterns and signals; ANNs are studied here in the context of groundwater remediation, using a flow and transport code to develop the training patterns and to validate the resulting management solutions (i.e., well locations and pumping schedules). The ANN is not programmed; it learns to solve problems by example. Under supervised learning, the ANN is "trained" on sets of data having known relationships. For many real world problems, precise solutions do not exist. In such cases, knowledge acquisition by example may be the only solution. In other words, if it is not possible to describe the logic behind a problem or predict behavior with analytical or numerical solutions to governing equations, traditional predictive analysis will be problematic. Yet ANN analysis may still be possible as it does not rely on predescribed relationships, but rather seeks its own relationships. One analogy that may be helpful here is to think of an ANN as a nonparametric, nonlinear regression technique. In traditional regression techniques a model must be decided upon a priori which the data will be fit to. The ANN approach is not as restrictive as the data will be fit with the best combination of nonlinear or linear functions necessary without the researher reselecting the form of these functions. A particular strength of the ANN approach is its ability to capture nonlinearities of the phenomena. When designed correctly, ANN's are stable, able to generalize, and can be relatively insensitive to noisy or spurious data [Dowla et al., 1990] These qualities make the ANN technology particularly attractive to many fundamental and applied problems which are complex, nonlinear, and poorly understood.

In this methodology, the flow and transport code develops the set of examples upon which the network is trained. The input to the ANN characterizes the different realizations of pumping, with each well having a discrete pumping level, such as on, off, or half on. The output is capable of characterizing the objectives and constraints of the optimization, such as whether regulatory goals have been met, cost functions and cleanup time have been minimized, and contaminant removal has been maximized. 
The supervised learning algorithm of backpropagation is used with the incorporation of the conjugate gradient method and weight-elimination procedures The conjugate gradient method is used to speed network convergence, and the weight-elimination procedures seek to improve network performance by penalizing network complexity. The network is first trained to predict a particular measure of the success of remediation. Determination of adequate training includes testing the network's performance on a group of pumping patterns which the network has not seen before. Then the trained network begans a search through various pumping realizations (i.e.; search through the feasibility space) making predictions about the success of remediation. The choice of patterns to evaluate is directed by a genetic algorithm, a simple yet robust search procedure based on the mechanics of genetics and natural selection. Note that in this approach the ANN has been trained to replace the flow and transport code. The necessary calls to the flow and transport code are all done in parallel at the beginning as an initial computational investment. The resulting management solutions are consistent with those resulting from a more conventional optimization technique, which combines solute transport modeling and nonlinear programming with a quasi-newtonian search. The results suggest that the ANN approach has the following advantages over the conventional technique for the test and application remediations: more independence of the flow and transport code from the optimization, greater ease incorporating hydrogeologic insight, and significant reduction of computational burden due to parallel processing of flow and transport simulations and recyling of these simulations. The ANN performance was observed upon variation of the problem formulation, network architecture, and learning algorithm. Field-scale applications and exploration of generalization capabilities are also presented.

The general structure of this dissertation is as follows. Section 2.0 presents background which the reader may find helpful in putting ANN groundwater management techniques into perspective. Important developments in groundwater management and ANN research are briefly summarized (Sections 2.1 and 2.2). Then, different types of learning algorithms are explored, including the backpropagation learning algorithm, which was used in this work (Section 2.3). 
Also included is a description of the optimization techniques used in the training and how network complexity may be limited to increase the networks' ability to predict patterns it has not seen before. Finally, the genetic algorithm is described (Section 2.4).

Section 3.0 describes how the ANN groundwater management methodology is implemented. A simple illustrative example is followed by a more complex remediation problem. Problem formulation and incorporation into a form which can be accessed by the ANN is described. The training and testing procedure for the network is presented as well as guidelines for the appropriate network architecture. The ANN solutions to these test remediations are compared to results from a more conventional technique of nonlinear programming.

Section 4.0 explores ways to increase understanding of how the network is making decisions for a particular problem. This involves dissection of the network weights and sensitivity analysis designed to determine the relative importance of the different inputs (i.e., wells).

Section 5.0 presents field-scale applications of the ANN groundwater management methodology. The field site used was the Lawrence Livermore National Laboratory in Livermore, California, which is a well-characterized Superfund site. Such objectives as maximizing mass removal, meeting regulatory constraints for off-site plume movement, time to clean-up, and cost of remediation are approached.

For the reader to whom ANN is a new subject, Section 6.0 discusses analogies between ANN and techniques which the reader may have some familiarity, such as regression and principal component analysis. The limitations of these analogies as well as the limitations and concerns of ANN design are also discussed. Section 7.0 is a summary and outline of possibilities for further work.

In addition to the main text, two appendices are included. Appendix A presents more detail on the learning algorithm of backpropagation with the conjugate gradient method used to speed learning. The conjugate gradient method adds a search direction conjugate with respect to the error surface when convergence slows down or the steepest descent search direction has been 
unsuccessful. Appendix B explains in more detail the conventional nonlinear programming approach to groundwater management. This should clarify how the calls to the flow and transport code are sequential. 


\subsection{Background}

\subsection{Groundwater Management}

The management of groundwater by optimization of the locations and pumping schedules of wells has been studied for some time. Common applications include maximizing conjunctive use [Bredehoeft and Young, 1970; 1983], meeting water-supply needs [Reichard, 1987], managing fluid storage [Reichard, 1987], optimizing dewatering [Aguado and Remson, 1974], and minimizing the effects of hazardous-waste disposal [Gorelick et al., 1984; Ahlfeldet al., 1986]. Early efforts in groundwater management considered water mass balances in economic and policy decisions. Examples of these early models are Chaudry et al. [1974] and Anderson et al. [1983]. Some of the first to advocate use of the governing equations of groundwater flow in groundwater management modeling were Bredehoeft and Young [1970, 1983] and Young and Bredehoeft [1972].

Groundwater management models that incorporate groundwater flow simulations are often referred to as simulation-management models. Gorelick [1983] describes three general categories of simulation-management models: (1) hydraulic management, (2) groundwater quality management, and (3) policy, evaluation, and allocation. Both hydraulic management models and groundwater quality management models are relevant to the design of aquifer remediation programs.

A myriad of groundwater remediation alternatives have been proposed to clean up our groundwater resources. Some examples are steam displacement [Hunt et al., 1988], purging by clean water [Bahr, 1989], and containment of contamination by hydraulic gradient [Atwood and Gorelick, 1985], grout curtains [Dougherty and Marryott, 1991], or biological curtains. It is common for remediation alternatives to include some form of pumping through extraction wells followed by treatment of contaminated water at the surface (i.e., pump-and-treat). Application 
of these remediation alternatives to a field site is usually preceded by appropriate modeling and predictive calculations. Groundwater management models have been used for some time to optimize locations and pumping schedules of extraction or injection wells in order to make restoration of contaminated aquifers more efficient [Willis, 1976; Gailey et al., 1991]. While not limited to the last application, this study focuses on problems of remediating or restoring contaminated aquifers.

. In most cases, pumping greater amounts of water adds to the expense of remediation. The sum of pumping rates is commonly used as the measure of remediation efficiency, or surrogate of costs, and is used as the objective of the optimization model, i.e., function to be minimized. The sum of pumping volumes is another common objective function. Objective functions of total pumping rates and total pumping volumes both assume a linear relationship between the amount of water pumped and the cost of remediation. The initial costs of constructing the remediation wells and treatment systems are often small relative to their operating costs throughout the cleanup process. Thus, the sum of pumping rates or pumping volumes is a reasonable and straightforward measure of remediation costs. However, this is a simplification of the problem. Other possible choices for the objective function are time of cleanup, pumping costs, well construction costs, water treatment costs, or some combination of these. One could also seek to maximize contaminant removal. The goal of the remediation is often incorporated into the constraint functions of the optimization model, or the functions that the objective function will be subject to. Possible remediation goals are hydraulic containment of the plume, complete removal of detectable contamination, and prevention of increases in downgradient contaminant concentrations above a chosen level.

Groundwater management research has addressed managing groundwater supply and minimizing the impact of waste disposal [Gorelick and Remson, 1982]. The first groundwater management models were restricted to linear equations [Aguado and Remson, 1974; Remson and Gorelick, 1980]. Finite difference approximations of the groundwater flow equation were used as constraints in a linear programing problem. Only the groundwater flow field was managed, and 
contaminant transport was not explicitly considered. Later, projections of contaminant transport were used in conjunction with linear programming to optimize injection and/or extraction wells in a pump-and-treat remediation scenario [Atwood and Gorelick, 1985; Walbridge, 1984]. Later still, nonlinear programming techniques were used to incorporate solute transport fully into the groundwater management methodology [Gorelick et al., 1984; Ahlfeld, 1987]. The nonlinear techniques were necessary because solute transport modeling of contaminant concentrations is dependent on the velocity of groundwater, which in turn is affected by the optimal pumping rates determined during optimization. Atwood [1984] and Walbridge [1984] both attempted to overcome the limitations of nonlinearity stemming from the dependency of the solute transport on the groundwater velocity. In 1984, Gorelick et al. investigated nonlinear optimization techniques that allow simultaneous consideration of hydraulics and solute mass transport. Jacobian matrices were used to determine the sensitivity of each constraint to changes in each decision variable. Gorelick et al. [1984] used perturbations in the pumping rates to evaluate the coefficients of the Jacobian matrix, in this case the sensitivity matrix of the effect of pumping on concentrations. Ahlfeld et al. [1986] also combined solute transport simulation and nonlinear optimization to address groundwater remediation, exploring similar as well as new management formulations, as outlined below.

Dogru and Seinfeld (1981) describe three methods for the evaluation of sensitivity coefficients: (1) the perturbation method, (2) the direct method, and (3) the adjoint sensitivity method. The perturbation method changes the value of the constraint or parameter of interest and observes how the system variables change [Gorelick et al., 1984; Ahlfeld, 1987]. The direct method involves differentiating the governing equations to determine how they change with respect to the system parameters. The adjoint sensitivity method employs a formulation similar to the previous methods for the sensitivity coefficient equations; however, it introduces a new set of variables, the adjoint states, and does not require as many simulations as the other two methods [Ahlfeld, 1987]. Ahlfeld et al. [1988a, 1988b] showed a computational savings of up to 
25 times through the use of the adjoint sensitivity approach for remediation of the Woburn Aquifer, Massachusetts.

Since the extension into the nonlinear regime, several nonlinear groundwater simulationmanagement models have appeared in the literature. Some work with nonlinearity stemming from hydraulics [Willis and Finney, 1985; Wanakule et al., 1986; Reichard, 1987], and others work with nonlinearity due to solute transport [Ahlfeld, 1987; Ahlfeld et al., 1986; Alley, 1986; Lefkoff, 1988; Lefkoff and Gorelick, 1985, 1986; Wagner and Gorelick, 1987; Wagner, 1988, Gailey et al., 1991]. Among the latter, Wagner and Gorelick [1987] and Gailey et al. [1991] have addressed stochastic nonlinear groundwater management methodology, which accounts for aquifer parameter uncertainty.

Concerns about the computational burden in field-scale applications, global optimality, and the difficulty of handling multiple objectives of different cleanup technologies (i.e., pumpand-treat as well as grout curtain, or pump-and-treat as well as electrical heating) have led some researchers to continue seeking improved methods of optimizing groundwater remediation. The original applications of nonlinear groundwater management [Gorelick et al., 1984; Ahlfeld, 1987] and most of the studies since then have used MINOS [Murtagh and Saunders, 1980] or NPSOL [Gill et al., 1986]. Both are well-known optimization algorithms that use the gradient method combined with a projected Lagrangian to manage nonlinear constraints. Ahlfeld [1987] found that cpu time increases nonlinearly (multiplicatively or exponentially) with the number of decision variables (such as possible well locations) and logarithmically as the desired cleanliness increases. The increased cpu time can be of particular concern when the groundwater system is modeled stochastically to quantify the effects of uncertainty in transport parameters and contaminant distributions [Wagner and Gorelick, 1987; 1989).

Neither MINOS nor NPSOL can guarantee a global optimum where the problem is nonconvex, which is the case for most groundwater field applications. In practice, once a solution is found, the algorithm is started again with variations on the initial value of the decision variable to gain some confidence that the solution is not a local one. The problem of 
global optimality is a complex one within the field of optimization and, in particular, groundwater management; there is great difficulty in guaranteeing that the optimal solution found is not a localized optimum. In general, nonlinear optimization algorithms seek a solution or point where the objective function is at a local minimum, providing that this point meets the constraints. If there were no constraints, an optimal solution would be found where the gradient of the objective function is zero. Constrained optimizations rely on a first-order Kuhn-Tucker point, which is defined as meeting certain conditions relating to the feasibility of the point and the existence of Lagrange multiplier vectors for bound and general constraints (Kuhn and Tucker, 1951; Powell, 1971 and 1974; Gill et al., 1986). These conditions aim to define a directional derivative at the point that is non-negative in each feasible direction. This indicates the point is indeed a local minimum with an increment in any feasible direction resulting in an increase in function value.

One of the mathematical premises of nonlinear optimization is that convex functions on convex sets have only one minimum value (Luenberger, 1984). One can envision a threedimensional function that is convex where any initial choice of a point on that function could be improved by moving in a downgradient direction to arrive at a point that is the local as well as global minimum. However, the management formulation of most groundwater management problems does not yield itself to a practical demonstration of convexity. Demonstration of convexity might involve computing the matrix of the second derivatives, the Hessian matrix, for all feasible values of the independent variables. Especially in a field-scale model, response of the concentration constraint functions to the pumping rates might not be convex. It is common is nonlinear groundwater management to assume that these functions are not convex and employ a method of varying initial values of the objective function. This method cannot guarantee global optimality; however, if the same optimal solution is found from starting the algorithm with different initial pumping rates, one can be more confident that the resulting optimal solution is effective on a larger, less-local scale. For more information on global optimization methods, the reader is referred to McCormick (1983). 
Both MINOS and NPSOL minimize a continuous and twice-differentiable function of the decision variables. Thus, in the common objective of minimizing cost, which is often some function of pumping rate summation, the cost function is viewed as continuous. If one wanted to add a discrete multiple objective, such as a slurry wall or biological curtain, mixed integer programming would be needed.

In light of these concerns, two approaches that have found recent success in other areas of combinatorial optimization-simulated annealing [Kirkpatrick et al., 1983; Rutenbar, 1989] and ANN- are of interest to examine for use in groundwater research. Combinatorial optimization refers here to problems involving minimization of a multiple-constraint objective function to determine a realization of optimal system parameters. Simulated annealing was developed by analogy to the statistical mechanics of annealing of solids; ANN was developed by analogy to the collective processing behavior of neurons in the human brain. Both techniques are believed to be robust methods with techniques to decrease chances of local minimum trapping of the objective function. The neural-network techniques have been more efficient in some applications [effrey and Rosner, 1986]. Both can perform discrete optimization (see Section 2.2.2 for further discussion of ANN and optimization) and thus have advantages when there are multiple technologies being optimized, such as whether a slurry trench will help or hurt remediation with pumping wells. In a discrete optimization problem, choices for pumping rates at a well would be discrete levels, e.g., "pump on" for rates between half-well and full-well capacities, and "pump off" at rates less than half-well capacity, or discrete levels between off and on at full-well capacity. This is an immediate simplification of the groundwater-management problem. In the continuous approach much of the computational effort goes into refining this pumping level. The potential yield of wells in field-scale problems is often low enough to be a binding constraint in the optimization. In other words, most geologic depositional environments, especially those comprised of alluvial, glacial, or fluvial sediments, have limitations in hydraulic conductivity. It is not uncommon for these wells to yield less than 100 gpm or even less than $50 \mathrm{gpm}$. Such small yield wells would commonly be operated at full yield 
to avoid more expensive small yield pumps and to clean up a field-scale site within a reasonable amount of time.

Considering the advantages of simulated annealing optimization, Dougherty and Marryott [1991] introduced and applied the simulated annealing methodology to groundwater management problems. In a hypothetical remediation example, results suggested that the relative cpu requirements for the simulated annealing approach increased at a slower rate than the gradient-based method of MINOS. A combined injection/extraction wells and slurry trench example was presented to demonstrate ease of incorporating multiple technologies.

Considering the advantages of neural networks, Ranjithan et al. [1990] trained a neural network to recognize particular patterns of spatial distribution of hydraulic conductivity that would be difficult to clean up. This neural network was promoted as a tool for decreasing the number of realizations of hydraulic conductivity, for instead of considering all possible realizations, only the difficult-to-cleanup patterns would be optimized. Hydraulic-gradient controls implemented by linear programming were used in test optimizations. The methodology of Ranjithan et al. [1990] uses linear programming optimization techniques and is still dependent on sequential calls to the flow and transport code.

The methodology in this dissertation does not address the issue of uncertainty and assumes a deterministic approach where the distribution of hydraulic conductivity is known. This is not a limitation of the methodology but rather the current evolution of applications thus far. It does address the nonlinear optimization problem of simultaneously considering contaminant transport and hydraulics. This optimization problem is labeled as nonlinear due to the dependency of contaminant transport on the groundwater velocities which are in turn dependent on the realization of pumping. The primary strength of this work lies in its freedom from the need for sequential simulations by the flow and transport code and the "recycling" potential for these simulations. Ultimately, some combination of the methodology of Ranjithan et al. [1990] and this work may allow both freedom from sequential calls to the flow and transport code and efficient incorporation of stochastic parameters. 


\subsection{ANN and Optimization}

Conventional computers are competent and fast at such tasks as number manipulation, data-base creation, and word processing. However, many problems in speech, robotics, pattern recognition, vision, and combinatorial optimization are not well-suited to their sequential approach. The ANN technology is an alternate computational approach based on new theories of the massive interconnection and parallel-processing architecture of biological systems. The ANN approach can be differentiated from the expert system approach of artificial intelligence in that the latter typically involves programming a system to apply a hierarchy of explicit rules. In contrast, ANN operates below a cognitive level of symbolic processing, e.g., the rules for all relationships and decisions cannot be written a priori. One might view expert systems as organizing behavior by description, whereas neural networks attempt to imitate the behavior. One might make a case that, instead of using a set of rules, human experts often apply intuition or deeper insight to the behavior that they have learned. Perhaps a better analogy is that an ANN is somewhat like a monkey trained to make the right decision without knowing the logic behind the answer. Of course the monkey has its own mind capable of action more complex than any task we might might train the monkey to do.

\subsubsection{Early Work}

Some have credited Freud with the initial work on artificial neuron systems [Simpson, 1990]. The first mathematical model of an ANN, the McCulloch-Pitts model, was based on the concept that neuron firing was an all-or-nothing process [McCulloch and Pitts, 1943]. This model presented the step threshold function as a transform mechanism for interconnected neurons to configure any logical function. Later work in the ANN technology was done by Rosenblatt $[1958,1962]$ on the perceptron and by Widrow and Hoff [1960] on the ADALINE. Rosenblatt demonstrated that a McCulloch-Pitts neuron [1943] could be trained to solve any linearly separable problem, i.e., separated by a hyperplane [Rumelhart et al., 1986], in a finite 
number of steps. He called this trained device, representing a neuron, a perceptron. Widrow developed the device ADALINE (ADAptive LINear Element, originally the ADAptive LInear NEuron), which can be used as a signal-processing filter. This device has a long history of successful applications, including echo elimination, automated control, and antenna-array adjustment. See Widrow and Stearns [1985] for a discussion.

With the book "Perceptrons," Minsky and Papert [1969] are often credited with causing pessimism about applications of the one- or two-layered perceptron networks and a dark age of ANN until the early 1980s. With the book "Parallel Distributed Processing: Explorations in the Microstructure of Cognition," Vols. 1 and 2, Rumelhart and McClelland [Rumelhart and McClelland, 1986, Vol. 1; McClelland and Rumelhart, 1986, Vol. 2] are often credited with leading the modern renaissance in ANN technology. Minsky and Papert were correct in their analysis of perceptron neural networks; however, the addition of more complexity in the networks, specifically in adding a middle (hidden) layer and a nonlinear transfer function to a multilayer perceptron network, together with a clear explanation of the backpropagation learning algorithm, overcame many of the limitations of the ANN.

\subsubsection{Optimization}

One of the first researchers to extend the application of neural networks into optimization was Hopfield [1984]. Later Hopfield and Tank [1985] applied a deterministic neural network architecture to solve NP-complete (nondetermined polynomial-time) constraint problems, such as traveling salesman problems and linear programming problems [Tank and Hopfield, 1986]. A predetermined energy function was used to model neuron interconnectivity. With the traveling salesman problem being a classic "intractable" problem, there is a growing body of Hopfield-Tank $(\mathrm{H}-\mathrm{T})$ formulations of the traveling salesman problem in the literature. The $\mathrm{H}-\mathrm{T}$ formulation is essentially a case of constrained quadratic minimization. There are some limitations and concerns with the $\mathrm{H}-\mathrm{T}$ traveling salesman problem algorithm, as it is sensitive to initial conditions and parametrization. Several researchers have explored heuristics designed to 
make the H-T algorithm more consistently generate valid tours [Clement et al., 1988; Szu, 1988; Hegde et al., 1988].

The Hopfield network was found to have difficulties capturing the nonlinearity of the groundwater applications in this dissertation. Also the inclusion of all aspects of the optimization into the energy function of the Hopfield network limited flexibility in pairing different objectives and constraints of remediation. In an alternate approach, the optimization problem was separated into two parts. The first part was training a multi-layer backpropagation network to predict the outcome of the flow and transport code. This involved an optmization to minimize the error between predicted and actual outcomes for a set of training examples. The optimization technique for this training was the conjugate gradient method, discussed further below. The backpropagation network had good success in being able to accurately capture the salient features of this extremely nonlinear problem and accurately predict the outcome of implementing various pumping realizations. Then the second part of the optimization problem was a search through possible pumping realizations to present to the trained backpropagation ANN. The optimization technique used to direct this search was a simple genetic algorithm. Through a randomized as well as historical process, this algorithm created new pumping patterns which were presented to the ANN to predict whether they would be improved solutions. The genetic algorithm will also be discussed in more detail below.

\subsubsection{Rapid Growth}

Since 1986 the variety of ANNs has rapidly expanded. In the now-classic "Parallel Distributed Processing" volumes, Rumelhart and McClelland described four types of ANNs. One year later, Lippman [1987] reviewed six types of ANNs at the First International Conference on Neural Networks. A few months later, 13 ANNs were described by Hecht-Nielson [1988]. In 1988, Simpson circulated a review of 26 types of ANNs that was later published as a book [Simpson, 1990]. In 1990, Maren et al. described about 24 ANNs, of which about 12 differ from those described by Simpson [1990]. Maren [1991] suggests that the rapid increase is continuing, 
with the number of ANNs now approaching 48. There are currently a vast array of ANN applications in the cognitive sciences, neurosciences, engineering, computer science, and the physical sciences. A review of these networks and applications is beyond the scope of this dissertation. Readers are referred to the review articles and reference books mentioned above for a complete discussion.

In the wake of this rapid growth in the field of ANN, there are differences of opinion and confusion about the best way to categorize and describe the various types of ANNs. Lippman [1987] distinguished between (1) supervised and unsupervised learning, and (2) binary (i.e., discrete) and continuously valued data. The difficulty of using these qualities as primary classification criteria is that many networks can be trained under supervised or unsupervised approaches (e.g., the Learning Vector Quantization Network and the Neocognitron), and use binary or continuously valued data (e.g., the discrete and adaptive H-T network) [Maren, 1991]. Simpson [1990] distinguished between supervised and unsupervised learning, as well as feedforward and feed-backward capabilities. Maren [1991] suggests first network structure, then dynamics, then learning as the gradation from coarse to fine ANN topology. She suggests that the expression "form follows function" applies in that the structure relates closely to what an ANN can be used for.

\subsubsection{Structure}

The basic processing elements of an ANN are the artificial neurons; however, they are more commonly referred to as nodes, units, or processing elements. An ANN node is not always analogous to a single biological neuron. Sometimes a node may represent the collective behavior of a group of neurons. For the sake of simplicity, a one-to-one correspondence between the biological and artificial neuron is used in Figure 2. The input pattern to the node, analogous to a stimulus to the dendrites of a biological neuron, may be represented as a vector of $N$ elements, $\mathbf{x}$, where $\mathrm{x}=\left(x_{1}, x_{2}, x_{3}, \ldots x_{N}\right.$. $)$. In groundwater management this vector might consist of pumping rates of different wells, hydraulic conductivity measurements, etc. Each input is 
(a)

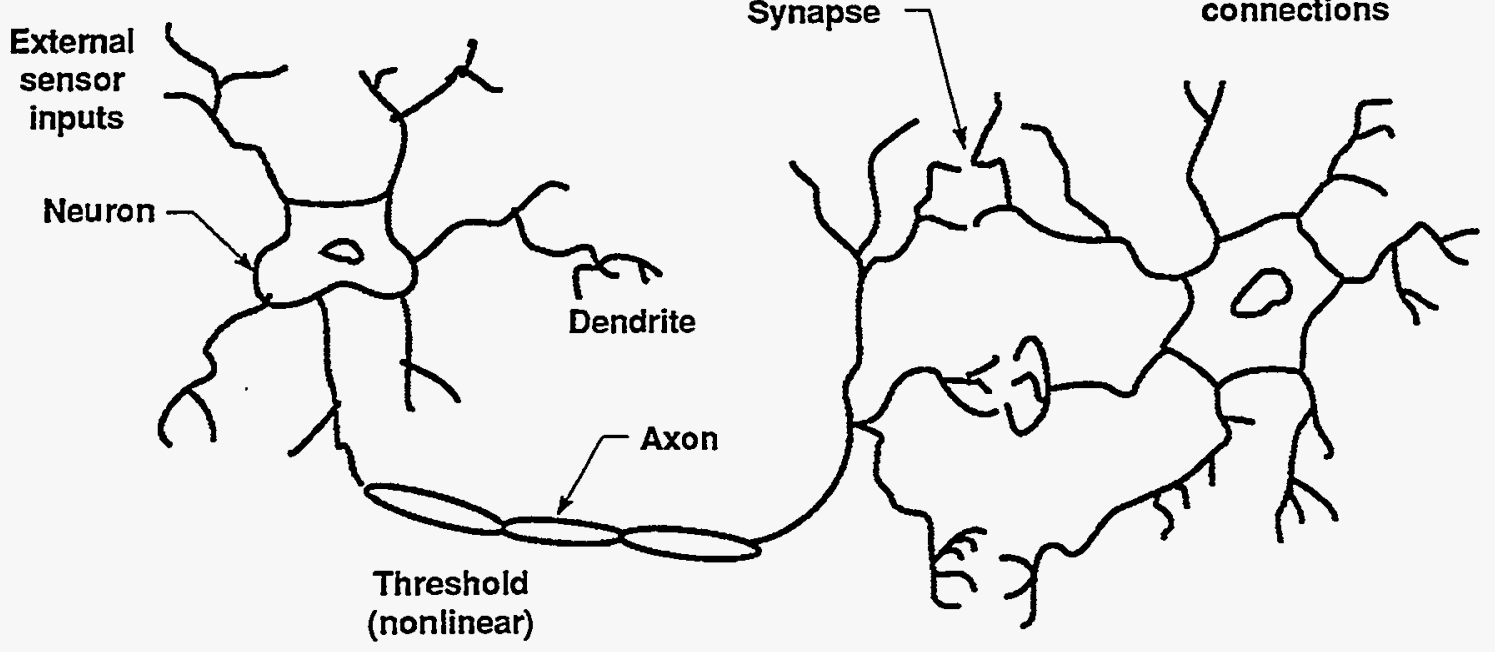

(b) Neuron

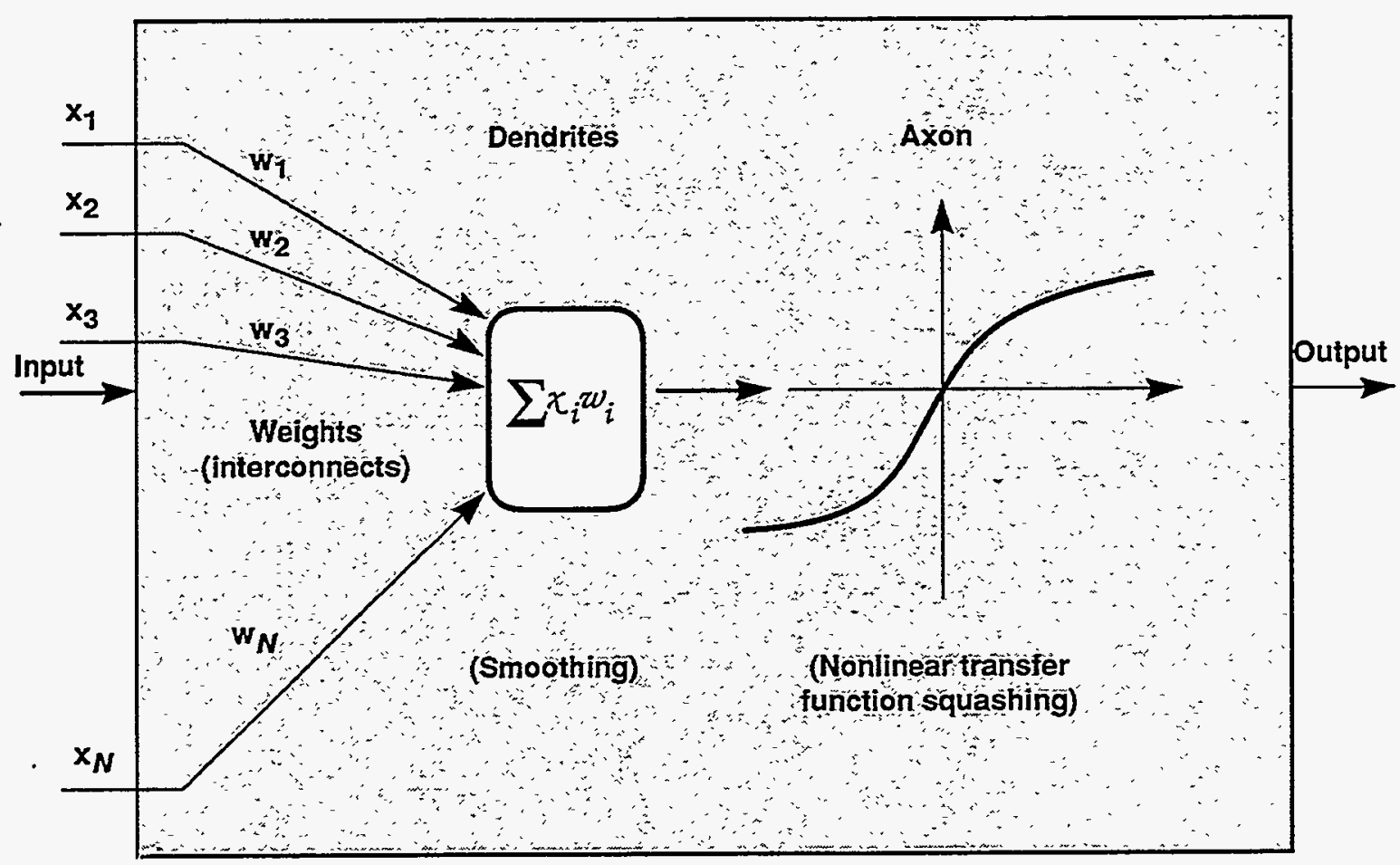

Figure 2. Neural networks: (a) Architecture of a biological prototype.

(b) Artificial digital prototype of a neuron. 
associated with a quantity called a weight or connection strength, which corresponds to the strength of the synaptic connection between biological neurons. The dot product of the inputs and their weights form an intermediate scalar, s, given by:

$$
s=\sum_{n=1}^{N} w_{n} \cdot x_{n}=\mathbf{w}^{\mathrm{T}} \mathbf{x}
$$

where $\mathbf{w}=\left(w_{1}, w_{2}, w_{3}, \ldots w_{N}\right)$ denotes the weight vector of the neuron. The vector dot product, $s$, is passed through a nonlinear transfer function (sometimes called a squashing function or activation function), $f($.$) , to yield the output, y=f(s)$, of the neuron for the input vector $x$. The output of a single neuron is a scalar; however, the output of a network of neurons can be a scalar or vector depending on the network architecture. The nonlinear transfer function is usually the sigmoid function defined by

$$
f(s)=\frac{1}{1+e^{-s}}
$$

The summation and nonlinear transfer function correspond to activity within the cell body, and the output corresponds to the firing frequency of the biological neuron. Note that the firing frequency of a biological neuron as a function of the input stimuli is well approximated by the nonlinear sigmoid function [Hopfield, 1982].

In her discussion of ANN topology, Maren [1991] suggests that the structure or architecture of an ANN can be described on three levels: micro, meso, and macro. In this approach, microstructure would describe the characteristics of each node in the network. Mesostructure would describe how the network is organized, including such features as (1) the number of layers, (2) the connection patterns, and (3) the flow of information. Macrostructure would describe how different networks are linked into interacting networks to accomplish different tasks. Starting with the fully connected McCulloch-Pitts model as a "formless" basic 
NN, she groups modifications of this structure into different classes of neural networks according to mesostructure. One of the simplest of these, the multilayer feed-forward network, is used in this study. The perceptron architecture exemplifies this class of mesostructure.

The multilayer feed-forward network imposes no restrictions on the number of inputs or outputs. In a multilayer network the output of one layer comprises the input to the next layer (Figure 3). The hidden layer may itself have more than one layer. If there are no feedback or lateral connections in the network, it is referred to as a multilayer feed-forward network.

The successful performance of a neural network is sensitive to its physical structure or architecture (i.e., number of input nodes, hidden-layer nodes, and output nodes). The appropriate architecture for an ANN is highly problem-dependent; however, there are some general guidelines and theory will be discussed later in this dissertation.

Knowledge learned by an ANN is encoded in the interconnecting weights of the network. The success of ANN learning can be thought of as the appropriate evolution of the interconnecting weights in order to result in the correct output for a given input pattern. It is the network weights which allow the input to be mapped into various decision regions of the solution space. In general an increase in the number of hidden nodes increases the ability of a network to frame more complex decision regions of the solution space. This is because each hidden node has the capacity to become a detector of a feature or relationship of inputs for the problem at hand. One might consider a numerical model as a predictor which is calibrated to some flow and transport problem by varying physical parameters to accurately reflect historical information. The ANN in this application is trained to be a predictor of a predictor (i.e., the numerical model). The numerical model preserves mass balance according to the numerical discretization of transport. During the learning process the ANN is provided the outcome of interest for different input patterns, i.e. pumping realizations. The ANN alters the magnitude of the handshaking between the nodes, i.e., network weights, in order to most accurately predict the outcome of all the pumping realizations of the training set. These weights then are a manifestation of the physical and spatial relationships between the well pumping and the outcome of interest. At a macro level these weights represent 


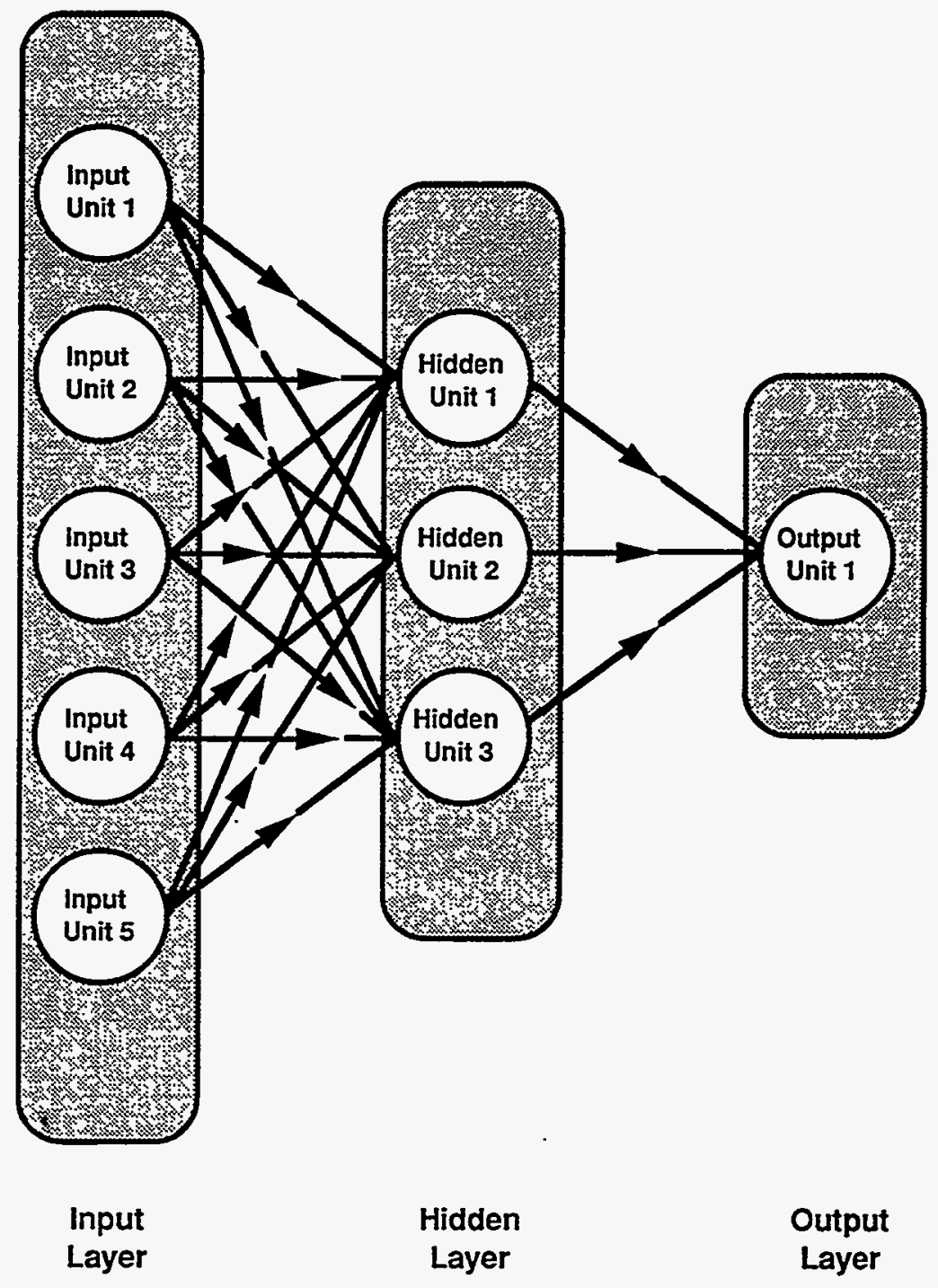

Figure 3. Structure of a multilayered neural network with a five-unit input layer, a three-unit hidden layer, and a one-unit output layer. Note that there are 18 weights in this network. 
the relationships of transport. The intricacies of these macro relationships may be approached by sensitivity analysis of the weights either by pertubation of the input values or weight values over a range of values. A discussion of learning follows in Section 2.3, and a discussion of macro relationships in remediation applications is discussed in more detail in Section 4.0.

\subsection{Learning}

Knowledge acquisition by ANNs is generally accomplished in one of three ways: unsupervised learning (i.e., self-organization), reinforcement, or supervised learning. Unsupervised learning does not rely on the ANN being provided with a desired response to the input or a performance score of the network's response. Instead, the ANN organizes its knowledge internally into categories which appear to have associated or collective properties. Reinforcement learning relies on a "tutor" grading the ANN's output responses to the training patterns. A high grade results in synaptic weight reinforcement, a low grade results in an adjustment of the weights to determine whether the grade can be improved. Supervised learning relies on the ANN being provided with the desired response to the training input patterns. This discussion focuses on supervised training because this learning approach allows the ANN to develop generalization or abstraction capabilities. There are some analogies between learning and a calibration process which will be discussed in more detail in Section 6.0.

Four of the major ANN learning algorithms are described here: Hebbian and neohebbian, Widrow-Hoff, perceptron, and backpropagation. The last was the learning algorithm used in this study, so it is treated in greater detail. For a discussion of learning algorithms the reader is referred to Dayhoff [1989], Hecht-Nielsen [1989], Wasserman [1989], Simpson [1990], and Murphy [1991]. 


\subsubsection{Hebbian Learning}

Hebb's learning law for the McCulloch-Pitts formal neuron proposes that the synaptic ties between neurons are strengthened between a neuron $A$ and a neuron $B$ when neuron $A$ persistently contributes to stimulating B when B is firing [Hebb, 1949]. Translated to ANNs, this law suggests that if neuron A consistently stimulates B while B is generating an output signal, the weight between A and B will increase in magnitude. Thus, during training of the ANN, neuron B will become more sensitive to neuron A's stimulus. The Hebbian learning law is an unsupervised learning algorithm.

Two difficulties with this law are (1) it allows the weights to grow without an upper bound, causing problems in practical applications, and (2) there is no method to assign negative (or inhibitory) weights between neurons. Many improvements have been made in the original Hebbian learning law over the past 40 years, including (1) a differential change to update the weights and (2) a change in the rate of learning in the weight update [Wasserman, 1989]. Biological systems can forget as well as learn, and the addition of controlling how quickly the network forgets [Grossberg, 1974; 1988] is sometimes referred to as neohebbian learning. The forgetting or extinction concept is implemented by Grossberg by breaking the weights and activation functions of the network into long and short term memory. While Hebbian learning is rarely seen in its pure form in ANNs today, its variations or modifications are common in many networks, including the instar and outstar neural systems of Grossberg [1974] and the drive reinforcement of Klopf [1987]; they even have a flavor of backpropagation, called backpropagation with recirculation.

\subsubsection{Widrow-Hoff Learning}

Widrow and Hoff [1960] developed the Adaptive Linear Element (ADALINE), which used a least-mean-squared (or Widrow-Hoff) learning algorithm to extend the computational model of a neuron. The errors in the neural network were minimized using steepest-descent optimization, a bias weight was added, and the Widrow-Hoff approach became the first 
supervised training algorithm. ADALINEs and MADALINEs (multiple ADALINEs) have been implemented extensively in digital electronics.

\subsubsection{Perceptron Learning}

In 1957, Rosenblatt presented the perceptron and introduced linear threshold logic to neural computing. Under this learning algorithm the output of the perceptron is 1 if the value of the weighted input is above or equal to some threshold, $q$, and 0 if it is less than q. Only synaptic weights associated with an incoming signal equal to 1 are changed. No change is made if the desired output response equals the actual output response. If the desired output response is equal to 1 and the actual output response equals 0 , then some fixed amount is added to the synaptic weight. If the desired output response is equal to 0 and the actual output response equals 1 , then the fixed amount is subtracted from the weight. While Widrow-Hoff learning is very similar to perceptron learning, it differs in that the perceptron learning treats only Boolean signals (i.e., following Boolean logic, if there is an even number of $1 \mathrm{~s}$ in a set of $0 \mathrm{~s}$ and $1 \mathrm{~s}$, then the output of the set is 1 , and if there is an odd number of $1 \mathrm{~s}$ in the set the output of the set is 0 ). In contrast, the Widrow-Hoff learning treats non-Boolean signals.

\subsubsection{Backpropagation Learning}

Werbos [1974] presented the "backpropagation learning algorithm," named for its propagation of errors backwards through the network to update the weights. His dissertation received little circulation, and the backpropagation learning algorithm was independently developed again and documented by two researchers in 1985 [Parker, 1985; Le Cun, 1985].

The backpropagation approach differs from the perceptron and the Widrow-Hoff approaches in the threshold or activation function. The backpropagation approach uses a differentiable function such as the sigmoid function, and the other two use the unit step function. The backpropagation algorithm makes use of robust optimization techniques to solve an unconstrained nonlinear optimization. The objective function $E(w)$ is minimized with respect to 
the independent variable $w$, the vector of network weights. $E(w)$ is the normalized sum of output errors over the entire training set and is mathematically defined by

$$
E(\mathbf{w})=\frac{1}{P} \sum_{p=0}^{P-1} E_{p}
$$

where $P$ is the number of patterns in the training set, and $E_{p}$ is the output error for the pth training pattern. $E_{p}$ is defined by

$$
E_{p}(w)=\frac{1}{2} \sum_{j=0}^{N-1}\left(T_{p j}-O_{p j}(w)\right)^{2}
$$

where $O_{p j}(w)$ and $T_{p j}$ are the network and the desired (or target) outputs, respectively, of the $j$ th output neuron for the $p$ th pattern, $O_{p j}$ is a function of $w$ emphasizing the dependence of the network output on the weight vector, $w$, and $N$ is the number of output neurons.

The weights are modified to minimize the error in the backpropagation algorithm according to the rule

$$
\mathbf{w} \leftarrow \mathbf{w}-\mu \nabla E(\mathbf{w})
$$

This rule states that the present weight is altered by an amount that is proportional to the negative derivative of the error with respect to that weight. The learning rate, a constant term, $\mu$, determines the rate at which the weight should be modified after each iteration.

The sigmoidal shape of the transfer or activation function has important implications for the effectiveness of the backpropagation learning. For most of the values of $s$, the value of the sigmoid function is close to one of its limiting values. Often the more positive asymptote is 1 , and the more negative asymptote is 0 or -1 . Let us consider -1 as the lower value for the rest of this discussion. Thus, the value of the transfer function is usually either high or low. Also the 
sigmoid function always has a positive derivative which is close to 0 for large absolute values of $\mathrm{s}$, and at a maximum when $\mathrm{s}$ is 0 . Note that in the backpropagation learning the changes to the weights are proportional to the derivative of the error (Eq.5), which is function of the value of the transfer or activation function (Eqs. 2 and 3). Note that this error is a continuous function whether the desired outputs (e.g., in this application, the objectives of remediation) are continuous or discrete. If the value of the derivative is near 0 , (i.e., when the value of the activation function is either large or small), the changes to the weights are small. And when the value of the derivative is at a maximum, (i.e., when the value of the activation function is in the middle range), the changes to the weights is much greater. These features are beneficial because the changes to the weights will be greatest when the activation function is in the middle range, and thus the derivative of the error is greatest. This will help force the weights more rapidly to their appropriate values.

The size of the learning rate parameter is an important issue in backpropagation learning and convergence. Too large a learning parameter can contribute to large oscillations in the weight modifications and abandonment of the training. Too small a learning parameter can contribute to very slow learning. Both of these extremes can lead to local error minimums and thus premature cessation of training. Incorporation of more refined optimization techniques can help to avoid such local error minima. Using Newton's method to adjust the weights eliminates some of the guesswork of choosing an appropriate learning parameter. Newton's method gives the gradient that will move from the current point on an $n$-dimensional curved error surface in a stright line to intersect the curved error surface at a lower value. Improvements in the backpropagation learning with optimization techniques is discussed further in Section 2.3.5.

The mechanics of the backpropagation learning algorithm are summarized as follows:

Step 1. Initialize the weights in the network. Set all weights in the network to some small positive and negative random values. Note that the backpropagation learning algorithm would not work if initial weights were all zero, and setting them to small random values assumes the mimimum possible about the system. 
Step 2. Present input and the actual outputs. The input can be a vector representing the well pumping rates, and a one-neuron output can represent, by 1 or 0 (i.e., a chosen threshold may be used to determine whether the output is closer to 1 or 0 ), whether regulatory constraints have been met. Note that in our groundwater application, actual refers to the results simulated for the flow and transport code.

Step 3. Compute the actual output of the network due to the current input presented to the network. This step is accomplished by simply forward-propagating the input vector through the network.

Step 4. Update the weights in the network. Compute the error using the network output and the desired output, and adapt the weights to reduce the error. The weight update rule, layer by layer, is the backpropagation learning algorithm. Starting from the output units of the network and working backwards layer by layer:

$$
w_{k} \leftarrow w_{k}-\mu x \delta_{k}
$$

where $\delta_{k}=f^{\prime}(s)(\partial E / \partial x)$, and $x$ is the input associated with the weight $w_{k}$. For the output units,

$$
\delta_{k}=O_{p k}\left(1-O_{p k}\right)\left[T_{p k}-O_{p k}\right]
$$

The term $\delta$ for a non-output layer unit can be derived in terms of the $\delta$ s of units whose inputs feed in from units in the previous layers, i.e., the outputs of the unit. For the hidden units the term is as derived in Dowla et al. [1990], namely

$$
\delta_{k}=f(s)(1-f(s)) \frac{\partial E}{\partial w_{k}}=f(s)(1-f(s)) \sum_{j} \delta_{j} \cdot w_{j}
$$


where $f(s)$ is the output of the hidden unit $k, s$ is the weighted sum of the inputs of the unit, and $j$ is over all units receiving input from the unit and scaling them with weights $w_{j}$. The $f($.$) used$ was the sigmoid function; however, these derivations could be used with other adjusted sigmoid functions or functions with similar shapes to the sigmoid function.

Step 5. Repeat by going back to step 2. This iterative process is continued until the error has been minimized. Learning consists of presenting to the network all elements of the training set and adapting the weights according to the rules described above. The process is repeated until the error at the output of the network meets the chosen convergence criteria for all the examples presented to the network.

In summary, each iteration of the backpropagation algorithm consists of two stages. In the forward-propagation stage, the input is propagated from the input layer to the output layer. In the backward-propagation stage, the error is propagated from the output layer to the input layer, and the weights are updated to reduce the error at the network output.

In 1986, Rumelhart and McClelland presented this learning algorithm and called it the delta rule [Rumelhart and McClelland, 1986]. A momentum term, a portion of the last gradient of the weight change, was also included in the weight update. Note that the Widrow-Hoff and backpropagation learning algorithms both rely strongly on classical optimization theory. The widespread use of ANNs in engineering and science applications today is related to the resurgence of the backpropagation learning algorithm. Convergence and learning issues of this approach are still being researched (see Section 6.0 for more discussion); however, the learning algorithm has been successful in a wide range of practical applications [Dowla et al., 1988, 1990; Weigend et al., 1990].

\subsubsection{Conjugate Gradient Method}

An increase in the number of weights in a network often increases the development of the network's inductive capabilities. This change encourages more extended and thus adaptable networks. For many problems, the number of weights in an ANN can be so great that the 
backpropagation learning algorithm becomes computationally demanding. One might consider reducing the size of the problem by preprocessing the data, using faster or more parallel computers, or applying some numerical optimization theory to speed up convergence of the backpropagation method. The latter approach has been used by several researchers. Becker and Cun [1989] used the quasi-newton method to approximate the second-order derivatives and speed up backpropagation convergence. They caution that the approximation may cause oscillations during convergence. Kramer and Vincentelli [1989] observed parallel implementations of conventional backpropagation with momentum, steepest descent, and the conjugate gradient technique with the Polak-Ribiere method [1969]. Their work suggests that the Polak-Ribiere method has advantages over the other two methods for small problems encoded as either a 0 or a 1 problems (i.e., Boolean) and for the problem of parity. The parity problem, often used for comparison purposes in the literature is a set of 0 s and 1s whose output is either 0 or 1 . An even parity problem would be defined as having an output of 1 if there were an even number of $1 \mathrm{~s}$ in the input set. An odd parity problem would be defined as having an output of 1 if there were an odd number of $1 \mathrm{~s}$ in the input set. Parity problems are also defined according to the number of components of the input set. Thus, a three-bit, even parity problem would be one with three inputs and an output of 1 if there were an even number of $1 \mathrm{~s}$ in the input.

Johansson et al. [1990] compared conventional backpropagation, steepest descent, and several conjugate gradient techniques in a broad range of numerical tests. The tests included three-, four-, and five-bit parity problems performed on ANN architectures with one and two hidden layers. They found that the conjugate gradient method speeded convergence in backpropagation learning by an order of magnitude relative to conventional backpropagation with momentum. In addition, they found that the conjugate gradient algorithm is less. vulnerable to instabilities and oscillatory difficulties of a fixed learning step size, as is the conventional backpropagation method. This study makes use of the work of Johansson et al. [1990] in employing a conjugate gradient method. A brief description of the conjugate gradient backpropagation algorithm is included in the Appendix; however, the reader is referred to 
Johansson et al. [1990] for details, including a tutorial introduction to the conjugate gradient method.

\subsubsection{Weight Elimination}

There is a second hazard of an increasing number of weights in addition to computational demand: the possibility of overfitting or overtraining of the network, which would cause a decrease in generalization performance (i.e., correct prediction of unseen test patterns) [Yves, 1990]. In overtraining, too many weights (degrees of freedom) of high precision can cause the network to begin to match the "noise" or idiosyncrasies in the training patterns, and, consequently, the network's capability to generalize to unseen cases without this "noise" decreases. Analogies to over-fitting problems in parameter estimation are discussed in Section 6.0. Weigend et al. [1991a] proposed a weight-elimination procedure for finding the minimal net by adding a network complexity penalty term to the backpropagation cost function. Inspired by minimization of the "description length" of a model [Cheeseman, 1990; Rissanen, 1989], Weigend et al. [1991a] give details on implementing this weight-elimination procedure.

In this procedure an error or cost function, $E$, may be defined such as that of Weigend et al. [1991a]:

$$
E=\sum_{k \in T}\left(\text { target }_{k}-\text { output }_{k}\right)^{2}+\lambda \sum_{i \in C} \frac{\frac{w_{i}^{2}}{w_{0}^{2}}}{1+\frac{w_{i}^{2}}{w_{0}^{2}}}
$$

where the first term measures the performance of the network, in this case the sum of squared error over the set of training patterns $T$. The second term measures the size of the network over all connections $C$. The parameter $\lambda$ represents the relative importance of the second term with respect to the first term. The parameter $w_{0}$ defines the scale of large and small weights; it is a free parameter of the weight-elimination procedure to be chosen by the user. 
The error function of this study is similar to that of the implementation of Jang et al.

[1991] :

$$
E=\sum_{k \in T}\left(\text { target }_{k}-\text { output }_{k}\right)^{2}+\lambda \sum_{i \in C} \frac{w_{i}^{2}}{1+w_{i}^{2}}
$$

Eq. 10 is a variation of Eq. 9; note that the parameter $w_{0}$ is not used. Let us look at the extreme values of $w_{0}$ to better understand its use in scaling large and small weights. If $\left|w_{j}\right| \gg w_{0}$; the penalty to the error function of a weight approaches one. If $\left|w_{j}\right| \ll w_{0}$; the penalty to the error function of a weight approaches zero. Thus the parameter $w_{0}$ encourages fewer but larger weights when it is small, and many small weights when it is large. Weigend et. al [1991a] suggest choosing $w_{0}$ of value one in networks where the activations are on the order of 1 . Another way to envision this is to graph the penalty function (Figure 4). The term weight elimination is somewhat confusing. For a given architecture the weights do not go away, but rather if their magnitude is strongly limited their impact on the solution is decreased. Encouraging the magnitude of all the network weights to be small is sometimes referred to as a subset of weight elimination called weight decay. Such a limitation is referred to in statistics as ridge regression [Weigend et al., 1991a, 1991b].

\subsection{Genetic Algorithm}

GAs have been established as a reasonable and robust approach to problems requiring efficient searches [Holland, 1992]. The reliance of GAs on analogies to biology and genetics limited their appeal for some time, but this is being overcome and many new applications are being seen in business, engineering, and science. The GAs have four advantageous features: 1) with a GA, the search begins from a population of parameter realizations, not a single realization 


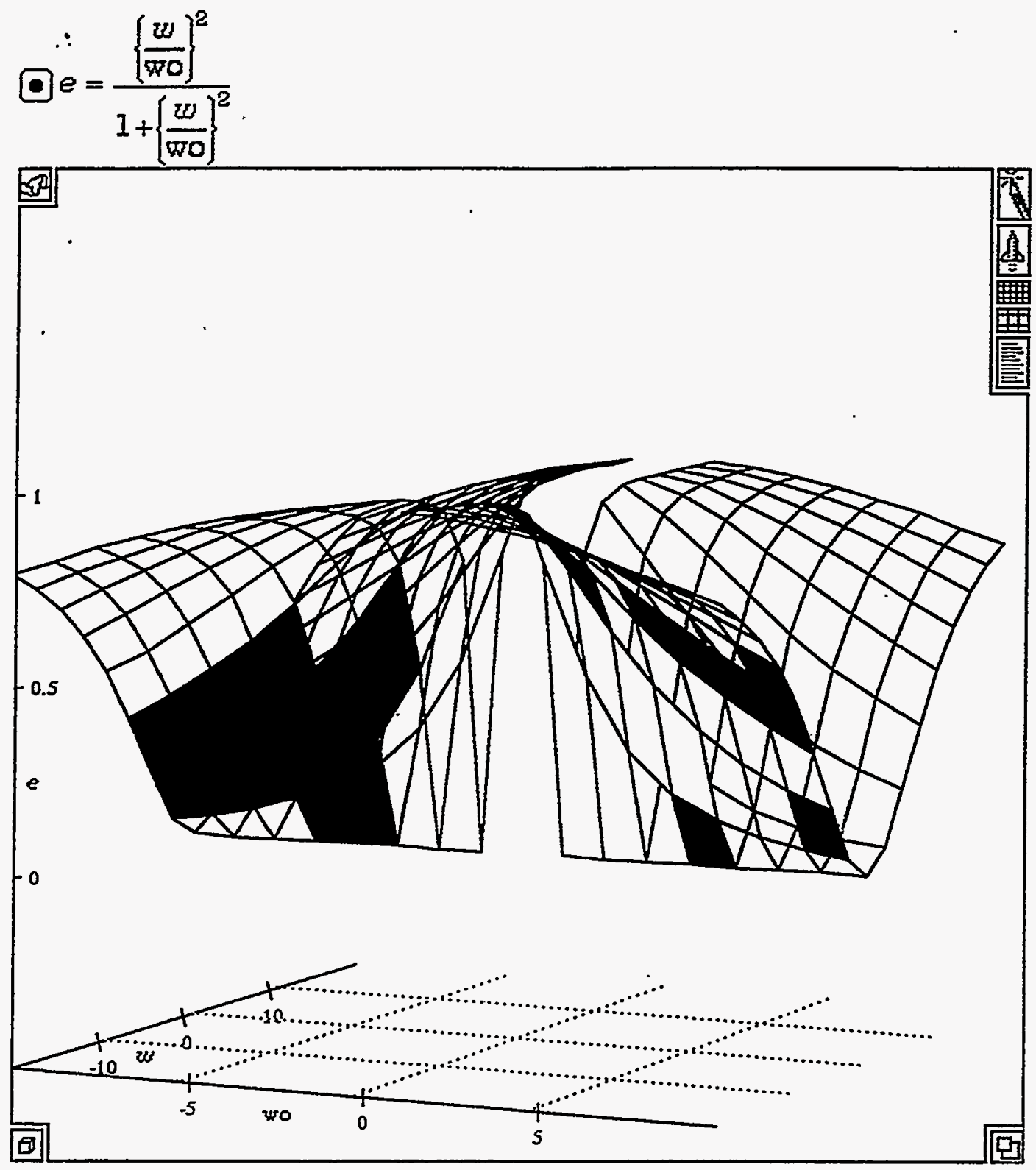

Figure 4. Weight elimination penalty function (on $\mathrm{z}$ axis) versus weight magnitudes (on the $y$ axis) and parameter wo (on the $x$ axis). 
as more conventional optimization procedures might. A realization refers here to one member of the set of possible configurations of the decision variables. 2) the GAs use probabilistic rather than deterministic rules for perturbation of the realizations. 3) the GAs manipulate a coding of the parameter set (i.e., decision variables) rather than the parameter set itself. In other words instead of perturbating a well pumping rate directly the GA would alter a pattern of $1 \mathrm{~s}$ and 0 s representing the pumping rates at all wells. 4) The objective function information is used directly in a GA rather than derivatives or other secondary descriptors. The latter characteristic is particularly handy for the ANN approach in this application as the value of the objective function is either known a priori (as the case with minimization of wells) or can be predicted rapidly with the trained ANN (Figure 5).

The three most basic operators of the GAs are:

1) Reproduction

2) Crossover

3) Mutation

Reproduction is an operator where parameter strings are copied according to their objective function values. This is often referred to by biologists as the fitness function in analogy to the Darwinian survival of the fittest. Goldberg [1989] uses the analogy of parameter strings being string creatures and instead of predators or pestilence, we use the objective function as the final arbiter of whether the string creature will survive. In other words the string creatures, or strings of parameter values, have a certain amount of information which may or may not be passed on to the next generation. The value of the objective function evaluated for that particular parameter realization will be important in deciding whether the information will survive.

Crossover is an operator to determine how the string creatures are paired up for mating and where the strings actually crossover. The crossover site between two mated strings becomes the point at which all the information on the first string before the site is paired with all the information on the second string after the site and vice versa (Figure 6). Let us give examples for these two operators before we discuss the third; mutation. 


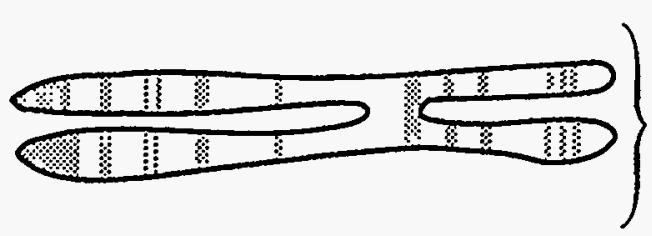

\section{Chromosome $\star$}

Gene (i.e. eye color) /

Locus (i.e. gene position)

allele (i.e. brown)
String Creature
Input function / Input location $1100 \quad 1 \quad 1 \quad 0$ input value

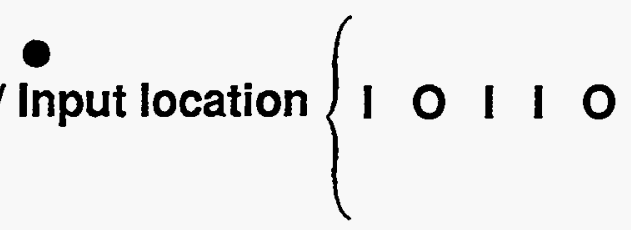
Well 1 Well 2 Well 3 Well 4 Well 5 Pumping Realization 4 on off on on off Well / location in String pumping level

Fitness measure $\star$ Objective function $\bullet$ quantification of success of remediation $\Delta$
Population / Genome $\star$

Set of string creatures set of pumping realizations $\Delta$

$$
\begin{array}{|l}
\text { Legend } \\
\star \text { Genetics } \\
\text { General programming } \\
\Delta \text { Groundwater application }
\end{array}
$$

Figure 5. The genetic algorithm: analogies. 


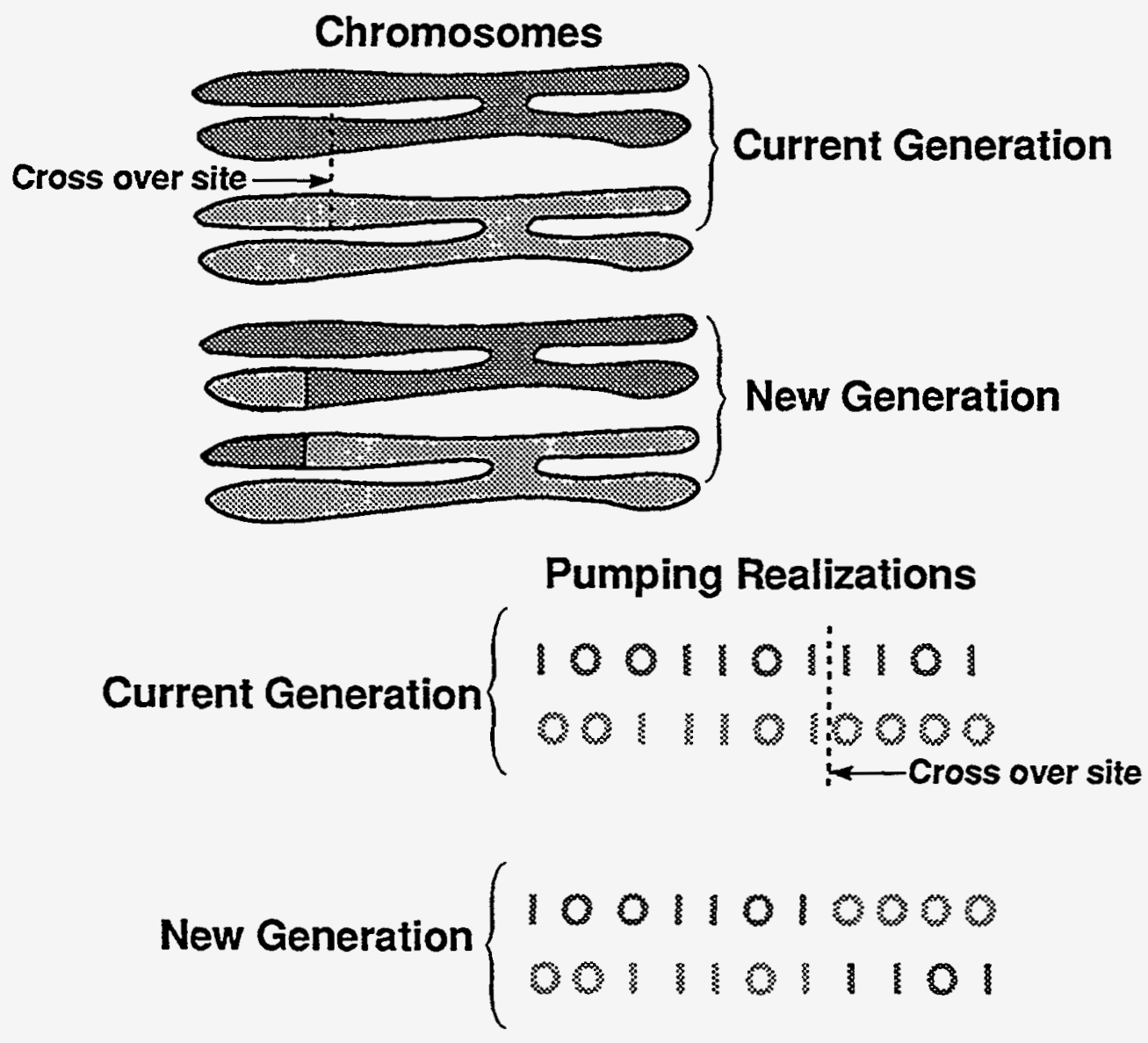

Figure 6. The genetic algorithm: crossover operator. 
One of the easiest ways to apply the reproduction operator is to sum the fitness of a population and calculate the percentage of population total fitness generated by each string creature. The percentage of that total fitness contributed by a specific string creature will determine how many times the creature gets into the mating pool, an intermediate step to the next generation. Let us consider the change of a population of 20 string creatures (Table 1a) from one generation to the next. In a groundwater management application an individual vector of parameters or realization of pumping could be analogous to an individual string creature. Let us further tailor this example to our application by considering each string creature to be a realization of pumping which consists of 20 inputs representing whether the 20 wells are on or off. The measure of fitness for this example will be the percentage of nonpumping wells. In other words a 1 would indicate no wells were pumping, .5 would indicate 10 of the 20 wells were pumping and 0 would indicate all wells were pumping. This measure of fitness would give a pumping realization a higher score if fewer wells were pumping. The current generation (Table 1a) has a total population fitness of 8.45 with each pattern of pumping having contributed between 3.6 and 5.9 percent. For simplicity, we will limit the next generation to the same total number of 20 patterns. Consider that if one pattern contributed at least 5 percent to the total, it should be able to be in the mating pool, i.e., if all had equal contributions each of the 20 patterns would have contributed 5 percent of the total fitness. We can establish a threshold heuristic so that if a pattern yielded more than 7.5 percent it would get to reproduce itself twice in the mating pool, and if a pattern yielded less than 2.5 percent of the total fitness it would not get to reproduce itself at all in the mating pool. In the current generation shown all patterns are able to reproduce themselves one time in the mating pool, and thus they each appear in Table $1 \mathrm{~b}$. 
Table 1. Transfer of information from one generation to the next according to the genetic algorithm for a population of $\mathbf{2 0}$ for the twenty-well remediation problem.

a) population of current generation.

\begin{tabular}{rlll}
\hline \multicolumn{2}{l}{ No. Pattern of Pumping } & $\begin{array}{l}\text { Fitness } \\
\text { Measure }\end{array}$ & $\begin{array}{l}\text { Probability } \\
\text { of Selection }\end{array}$ \\
\hline \hline 1 & 00101010011111000110 & .50 & .059 \\
2 & 01001010011111000110 & .50 & .059 \\
3 & 01101000011111000110 & .50 & .059 \\
4 & 01101010011110000110 & .50 & .059 \\
5 & 01101010011111000100 & .50 & .059 \\
6 & 00001000111111010110 & .50 & .059 \\
7 & 11011011011011011011 & .30 & .036 \\
8 & 01101010011111110101 & .35 & .041 \\
9 & 10011001101110011101 & .40 & .047 \\
10 & 10011001111110011101 & .35 & .041 \\
11 & 11011011011110000110 & .40 & .053 \\
12 & 11011111101110000110 & .35 & .041 \\
13 & 00001100111111010110 & .45 & .053 \\
14 & 00001011111111110110 & .35 & .041 \\
15 & 01001100101111010110 & .45 & .053 \\
16 & 01101010011111000110 & .45 & .053 \\
17 & 01001110011111000110 & .45 & .053 \\
18 & 01101010111111000110 & .40 & .047 \\
19 & 01001110011111100110 & .40 & .047 \\
20 & 11011111011110000110 & .35 & .041 \\
& Total Fitness 0f Population & & 8.45
\end{tabular}

b) mating pool after reproduction,

\begin{tabular}{|c|c|c|c|}
\hline & & $\begin{array}{l}\text { Random } \\
\text { Mate }\end{array}$ & $\begin{array}{l}\text { Random Crossover } \\
\text { Site }\end{array}$ \\
\hline 1 & 00101010011111000110.50 & 7 & 1 \\
\hline 2 & 01001010011111000110.50 & 6 & 4 \\
\hline 3 & 01101000011111000110.50 & 8 & 6 \\
\hline 4 & 01101010011110000110.50 & 5 & 7 \\
\hline 5 & 01101010011111000100.50 & 4 & 7 \\
\hline 6 & 00001000111111010110.50 & 2 & 4 \\
\hline 7 & 1101101101101101101130 & 1 & 1 \\
\hline 8 & 01101010011111110101.35 & 3 & 6 \\
\hline 9 & 10011001101110011101.40 & 13 & 7 \\
\hline 10 & 1001100111111001110135 & 18 & 3 \\
\hline 11 & 11011011011110000110.40 & 17 & 11 \\
\hline 12 & 11011111101110000110.35 & 15 & 6 \\
\hline 13 & 00001100111111010110.45 & 9 & 7 \\
\hline 14 & 00001011111111110110.35 & 16 & 15 \\
\hline 15 & 01001100101111010110.45 & 12 & 6 \\
\hline 16 & 01101010011111000110.45 & 14 & 15 \\
\hline 17 & 01001110011111000110.45 & 11 & 11 \\
\hline 18 & 01101010111111000110.40 & 10 & 3 \\
\hline 19 & 01001110011111100110.40 & 20 & 8 \\
\hline 20 & 11011111011110000110.35 & 19 & 8 \\
\hline
\end{tabular}


Table 1 continued

c) example of crossover operator for first two matings,

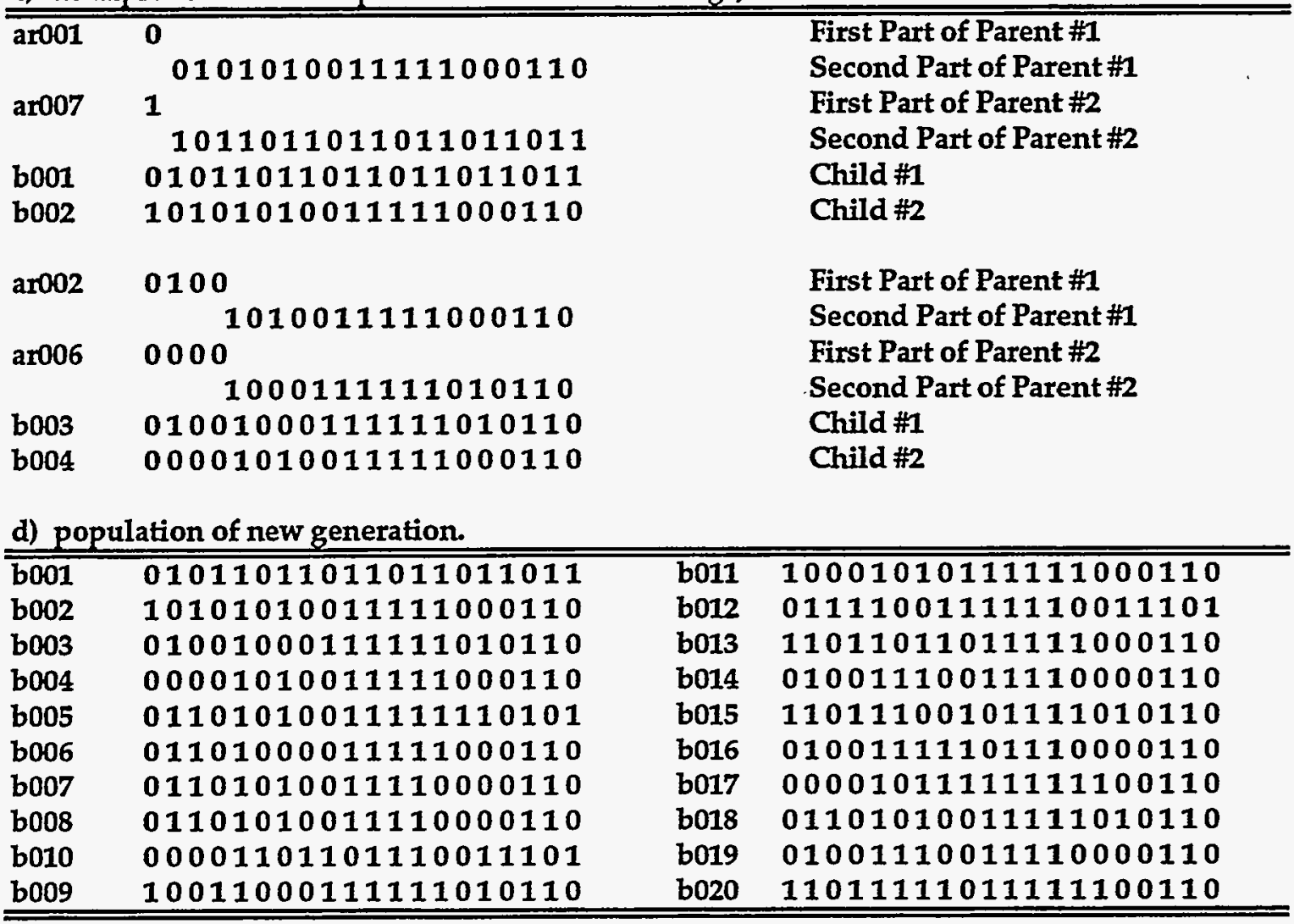

This mating pool becomes a group of string creatures looking for mates. The mating or crossover can be simply performed in two steps: 1) random selection of a mate, and 2) random selection of a crossover site for this mating. We see that pattern 2 of the mating pool has drawn pattern 6 as a mate with a crossover site of 4 . So the first four variables of pattern 2 are mated to the last 16 variables of pattern 6 and the first four variables of pattern 6 are mated to the last 16 variable of pattern 2. These two new patterns enter the new generation.

The third operator, mutation, is surrounded by some controversy in genetics. Mutation is designed to avoid the loss of valuable genetic material, which theoretically may result from reproduction and crossover [Holland, 1992]. Although mutation is one of the most familiar terms in genetics, its role in the GAs is often misconceived. Its role is not to generate new structures or patterns, the crossover and reproduction operators do that nicely. Mutation's primary and unique role is to create a mechanism by which information or small segments of parameter 
strings, can be reintroduced into a population. In the simple GA, mutation is a random alteration of a variable in a string. Generally there is a small probability of mutation occuring. Goldberg [1989] suggested a reasonable mutation probability may be .001 . There are $20 * 20$ possible mutation sites in this population, so the expected muations would be $20^{*} 20^{*} .001=.4$ bits of information. Since the minimum bit that can be changed is 1 , no mutations were expected in a population this small and none were simulated. However, one might randomly choose a mutation site bit once every second or third generation.

These three operators encourage persistence of patterns judged to be "most fit" asthe population of patterns evolve from one generation to the next. The GA then directs a path through possible patterns seeking new patterns which may be more optimal or "more fit". The GA will continue to include in the population of patterns the most optimal which it has discovered and throw out the less optimal in its search for the best patterns. 


\subsection{Groundwater Management Modeling using ANNs}

In this section, the details of implementing a groundwater management model with ANN and GA are given. The methodology is first presented for a groundwater remediation problem involving three wells where the total number of combinatorial pumping realizations are only eight. Combinatorial refers to the possible combinations of remediation wells which make up the different pumping realizations. In the three-well example, considering only on or off (i.e., only 2 possible states) for the 3 wells, there are $2^{3}$ or eight possible pumping realizations. Later a twenty-well remediation problem is addressed. The combinatorial dimension of this twenty-well problem, again considering only extraction at well yield (i.e., full on) or off, is $2^{20}$, or over one million possible pumping realizations. The ANN groundwater management approach seeks an efficient search through these possible realizations using the trained ANN for a quick evaluation of the remediation objectives and/or constraints.

\subsection{Implementation of ANN Groundwater Management Model}

The implementation of the ANN groundwater management methodology developed here can be generalized into six steps:

(1) Conceptualization and formulation of the management problem; choice of objective functions and constraint functions.

(2) Incorporation of the management formulation into components that can be represented by a particular type of ANN network.

(3) Groundwater flow and transport simulations (in parallel) to development training and testing patterns. 
(4) Training of the ANN; alteration of architecture to yield maximum performance of the ANN.

(5) Search through possible pumping realizations, i.e., management solutions, with the GA to find optimal realization. Use the trained network to predict the value of objective and/or constraint functions of the possible combinatorial pumping realizations.

(6) Using the flow and transport code to confirm that the recommended management solutions work.

\subsubsection{Illustrative Example: Three-well Remediation Problem}

A simple three-well example is given to illustrate how the objectives and constraints of a remediation problem can be approached with an ANN. Let us consider a case in which three possible remediation wells may be pumped to prevent a contaminant plume from moving westward (Figure 7). The single objective of the ANN groundwater management model is to pump the minimum volume of water from the aquifer subject to the constraint of keeping concentrations in hydraulically downgradient monitor wells equal to or below a regulatory limit of $20 \mathrm{ppb}$. The formulation of the groundwater management model may be expressed mathematically as:

$$
\begin{array}{cl}
\text { Minimize } & m \sum_{i=1}^{J} q_{i} \\
\text { subject to } & c_{j} \leq c_{\max } \text { for all } j \\
\text { either } q_{i}=q_{\max } \\
\text { or } q_{i}=0
\end{array}
$$


(a)

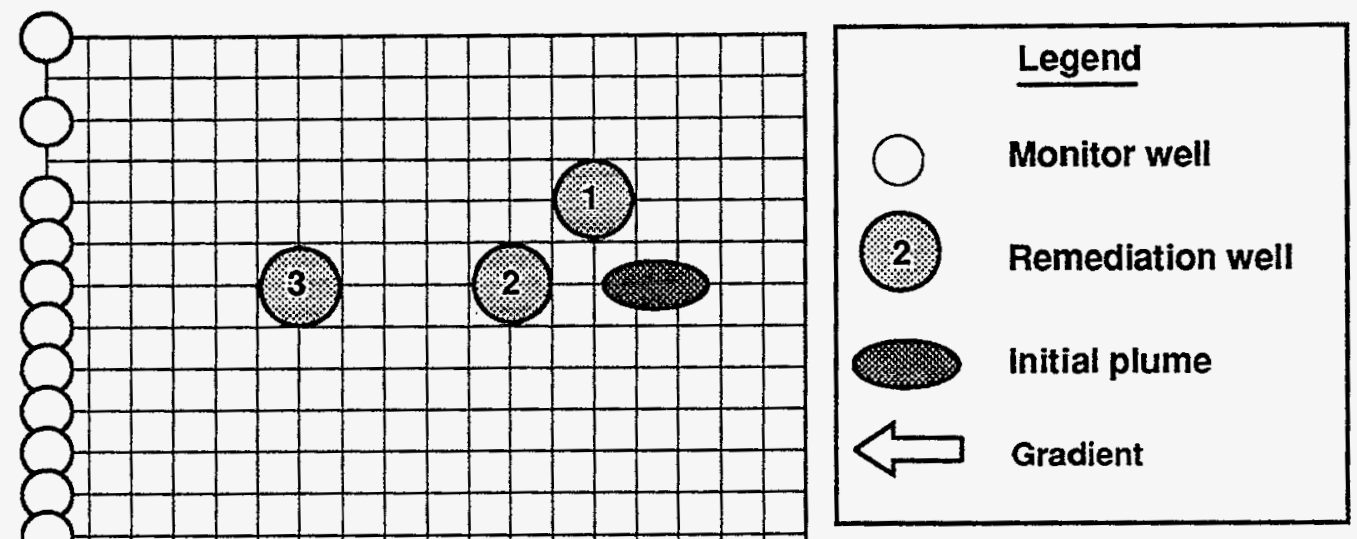

(b)

Pumping realization (pumping well \#3 only)

\section{Input vector}

Hidden layer
(3 nodes)

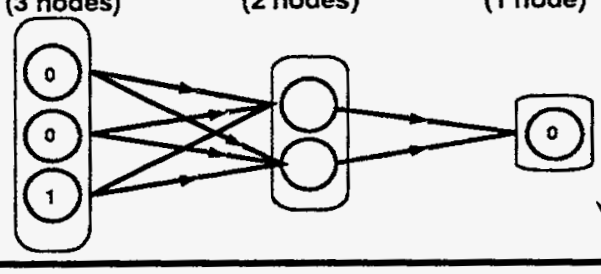

Did pumping realization successtuliy remediate? If output $=1$ Yes If output $=0$ No

(c)

\begin{tabular}{|c|c|}
\hline Input & Output \\
\hline 000 & 0 \\
\hline 100 & 0 \\
\hline 010 & 1 \\
\hline 001 & 0 \\
\hline 110 & 1 \\
\hline 011 & 1 \\
\hline 101 & 0 \\
\hline 111 & 1 \\
\hline
\end{tabular}

Figure 7. Illustrative example of ANN groundwater remediation. (a) Numerical simulation grid with three possible pumping wells downgradient from a contaminant plume and upgradient from a line of monitor wells. (b) Mapping from conceptualization to a decision on remedial success. (c) ANN input and output patterns for all eight possible pumping realizations. 
where $m$ is the length of the planning horizon, $J$, is the number of nodes that can be pumped as remediation wells, $q_{i}$ is the well pumping rate at location $i, c_{j}$ is the concentration of contaminant at monitor well $j, c_{\max }$ is the target concentration or action level at the monitor wells, and $q_{\max }$ is the maximum pumping capacity at the remediation wells.

A north-south line of fourteen monitor wells is introduced down-gradient from the plume as control points to observe contaminant concentrations. The three possible remediation wells are located between the monitor wells and the initial plume. From a combinatorial optimization perspective, this is an extremely simple example. If we consider only the discrete possibility of each well being either on or off, there are only $2^{3}$ possible realizations of pumping.

To incorporate the management formulation into components that can be represented by an ANN network, let us consider an input vector of three components to represent a realization of pumping (Figure $7 \mathrm{~b}$ ), with a 1 indicating that the well is on at full capacity and a 0 indicating that the well is off. The output will represent whether the pumping realization was successful (1) or not $(0)$ in meeting the regulatory constraint (Figure $7 \mathrm{c}$ and Table 2).

Table 2. Illustrative example. (a) Input and output patterns

\begin{tabular}{|c|c|c|c|c|c|c|}
\hline \multicolumn{4}{|c|}{$\begin{array}{l}\text { Input: } \\
1=\text { pumping at } 35 \mathrm{gpm} \\
0=\text { off }\end{array}$} & \multicolumn{2}{|c|}{$\begin{array}{l}\text { Highest Concentration } \\
\text { over all Time Steps } \\
(\mathrm{ppb})\end{array}$} & $\begin{array}{c}\text { Output: } \\
1 \text { if } c_{\max }<20 \mathrm{ppb} \\
0 \text { if } c_{\max }>20 \mathrm{ppb}\end{array}$ \\
\hline 0 & 0 & 0 & & 21.95 & & 0 \\
\hline 1 & 0 & 0 & & 21.90 & & 0 \\
\hline 0 & 1 & 0 & & 19.99 & & $1^{a}$ \\
\hline 0 & 0 & 1 & & 21.10 & & 0 \\
\hline 1 & 0 & 1 & & 21.00 & & 0 \\
\hline 1 & 1 & 0 & & 19.96 & & 1 \\
\hline 0 & 1 & 1 & & 19.15 & & 1 \\
\hline 1 & 1 & 1 & & 19.11 & & $\mathbf{0}$ \\
\hline \multicolumn{7}{|c|}{$\begin{array}{l}\text { aOptimal solution. } \\
\text { (b) ANN performance. }\end{array}$} \\
\hline $\begin{array}{l}\text { Architecture } \\
3-2-1 \\
3-2-1\end{array}$ & $\begin{array}{l}\text { No. of } w \\
11 \\
11\end{array}$ & & $\begin{array}{l}\text { No. } 0 \\
3 \\
4\end{array}$ & examples & $\begin{array}{l}\text { Accuracy } \\
100 \% \\
100 \%\end{array}$ & $\begin{array}{l}\text { Generalization } \\
60 \% \# \\
100 \% @\end{array}$ \\
\hline
\end{tabular}

\# This network was trained with the second, sisth, and eighth pattern listed in (a).

Q This network wast trained with the second, fourth, sixth, and eighth pattern listed in (a). 
The training and testing patterns are developed with the aid of a 2-D hybrid flow and transport model [Voss, 1984] discussed in more detail below (see Section 3.1.2). All eight of the possibilities can be run to find that the optimal solution would be to pump Well No. 2 only. The flow boundaries on the numerical mesh are no flow on the northern and southern boundary and constant head on the eastern and western boundary; the direction of groundwater flow is directly westward. The hydraulic conductivity is homogeneous over the model area. The potential yield of the wells is assumed to be $35 \mathrm{gpm}$, which is a midvalue of the 5 to $70 \mathrm{gpm}$ actual yield range of wells completed in a field site with similar gradient and average hydraulic conductivity (Thorpe et al., 1989).

An ANN was trained with a backpropagation architecture of 3-2-1, i.e., 3 input values in each input pattern, 1 hidden layer with 2 nodes, and 1 output value. The network could accurately predict whether or not a pumping realization would be successful after learning only four of the eight possible patterns. The example patterns used to train the network are listed in Table $2 \mathrm{~b}$. Note that after being trained on three patterns the network only predicted the correct output for the unseen patterns $60 \%$ of the time i.e., 3 or 5 . After being trained on four patterns, the network correctly predicted the output for all four remaining patterns. This problem converged rather nicely despite the fact that there were only 4 input patterns to train on and 11 weights in the architecture. A rule of thumb is that one would like at least as many examples to train on as there are weights in the network. Some researchers suggest the number of training examples should be 5 to 10 times the number of network weights [Tishby et al., 1989; Maren et al., 1990] Thus, after training the network on four input patterns that did not include the optimal input pattern, the network was able to accurately predict which pumping realizations would meet the regulatory constraints; and postprocessing was done to determine which of the successful realizations involved the smallest number of wells. In this simple case, we knew the answer before we started training the ANN, as we had run all eight possible realizations of pumping. Thus, no directed search through the possible combinations of pumping patterns or 
additional runs of the flow and transport code were needed to validate the recommended solutions.

A more conventional nonlinear groundwater management model was applied to this simple example that incorporated both nonlinear programming with a quasi-newton search [Gill et al., 1986] and the same solute transport model. Remember that the comparison is not exact because the nonlinear programming seeks to minimize a continuous function. Thus, some simplification is immediately possible in the discrete approach to optimization. However, it should be noted that the nonlinear programming approach involved calling the flow and transport code 26 times to determine an optimal management solution consistent with the results of the ANN management model. It was recommended that well No. 2 be pumped at a level of $34.9 \mathrm{gpm}$ as the minimum pumping rate necessary to meet the regulatory constraints.

\subsubsection{Solute Transport Modeling}

This methodology is not limited to the type of solute transport modeling employed, but a brief description of the latter is included for clarity. This study employs the advection-dispersion approach to solute transport modeling. Solutes are transported through a porous medium by advection of the fluid and molecular diffusion. Variations of the fluid velocity within the pore spaces result in mechanical dispersion. Traditionally, the flux due to mechanical dispersion and molecular diffusion are combined and called hydrodynamic dispersion. The term for the mass flux, composed of the advective flux and dispersive flux, is then combined with a continuity or conservation of mass equation to derive the advection-dispersion equation. The contamination was modeled as being conservatively transported; however, the model is capable of addressing sorption of a compound onto or from the formation's solid matrix with the addition of a source/sink term to the advection-dispersion equation.

The groundwater simulations are based on the governing equations for steady, confined ground water flow and transient solute transport (see Bear, 1972, 1979): 


$$
v=\frac{-T}{\varepsilon} \cdot \nabla h
$$

$$
\nabla \cdot(-\mathbf{T} \cdot \nabla h)-q=S_{S} \frac{\partial h}{\partial t} b \text {, and }
$$

$$
\frac{\partial \varepsilon c}{\partial t}+\nabla \cdot(\varepsilon v c)-\nabla \cdot(\varepsilon \mathrm{D} \cdot \nabla c)+\mathrm{Q}\left(c^{\prime}\right)=0
$$

where

$v=$ ground water velocity vector, $v(x, y), \mathrm{m} / \mathrm{s}$;

$\mathrm{T}=$ transmissivity tensor, $\mathrm{T}(x, y), \mathrm{m} / \mathrm{s}$, equal to $\mathrm{Kb}$;

$b=$ aquifer thickness, $m$;

$\mathrm{K}=$ hydraulic conductivity tensor, $\mathrm{K}(x, y), \mathrm{m}^{2} / \mathrm{s}$;

$\varepsilon=$.volumetric porosity, $\varepsilon(x, y)$, dimensionless;

$h=$ hydraulic head, $h(x, y), \mathrm{m} ;$

$S_{S}=$ specific storage, $1 / \mathrm{m}$;

$Q=$ fluid sinks(t)/sources $(-), Q(x, y), \mathrm{m} / \mathrm{s}$;

$c=$ solute concentration, $c(x, y, t), \mathrm{mg} / \mathrm{L}$;

$c^{\prime}=$ concentration of solute in fluid source $/ \operatorname{sink}, c^{\prime}(x, y, t), \mathrm{mg} / \mathrm{L}$;

$\mathrm{D}=$ hydrodynamic dispersion tensor, $\mathrm{D}(x, y, \mathrm{v}), \mathrm{m}^{2} / \mathrm{s}$;

and

$$
\mathrm{D}=d_{m} I+\mathrm{M}
$$

where 
$d_{m}=$ molecular diffusivity of solute in saturated porous media, including tortuousity effects, $\mathrm{m}^{2} / \mathrm{s}$;

I = identity tensor, dimensionless;

$\boldsymbol{M}=$ macrodispersion tensor, $M(x, y, v), \mathrm{m}^{2} / \mathrm{s}$.

Darcy's law [Eq. (15)] is combined with a fluid mass balance to describe transient flow in a confined aquifer [Eq. (16)]. Its solution determines the hydraulic head distribution for a particular set of boundary conditions and system stresses. Equation (17) is a solute mass balance for a confined aquifer under transient conditions. Spatial changes in solute concentration are described by the solution of Eq. (17). The solution of Eq. (15), the head distribution $h(x, y)$, is used in Eq. (16) to determine fluid velocities $v(x, y)$. The velocities in conjunction with boundary conditions and time-dependent source concentrations allow Eq. (17) to then be solved for transient concentration distribution $c(x, y, t)$.

\subsection{The Twenty-well Remediation Problem}

\subsubsection{Conceptualization and Formulation}

Let us consider, as a hypothetical test remediation, multiple contaminant plumes moving westward across a heterogeneous field site, which causes a complex transport scenario (Figure 8). The goal of remediation is to keep contaminant mass concentrations from moving off the site, i.e., past a chosen north-south line of monitor wells in amounts that are above a regulatory concentration constraint. The measure of efficiency of the remediation (i.e., objective) is to minimize the summation of pumping volumes of the remediation wells. There is only one management period over the planning horizon, i.e., the pumping rates will be kept constant 

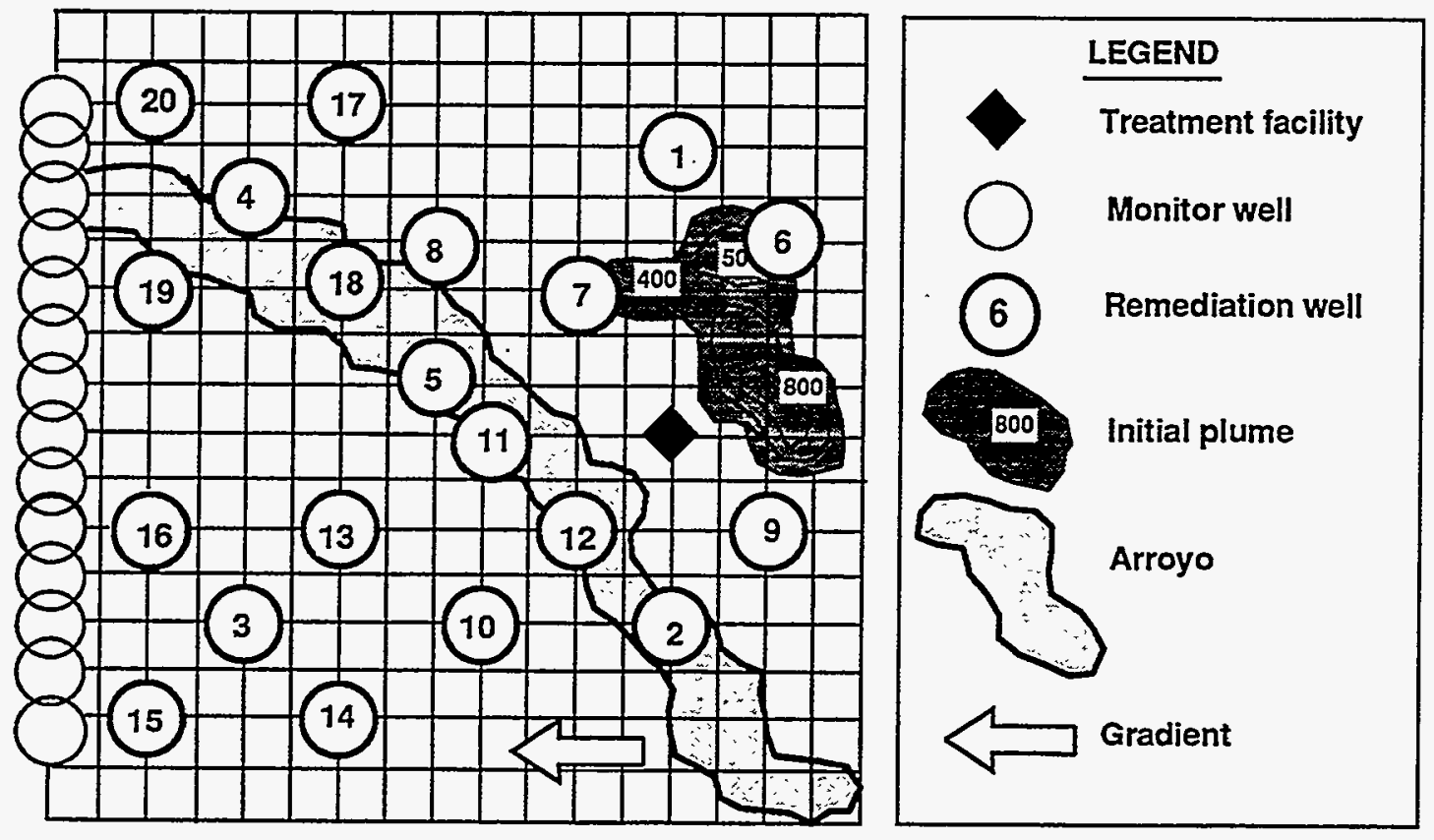

\begin{tabular}{|c|c|c|c|c|c|c|c|c|c|c|c|c|c|c|c|c|c|c|c|c|c|}
\hline \multicolumn{22}{|c|}{ Input variables } \\
\hline 1 & 2 & 3 & 4 & 5 & 6 & 7 & 8 & 91 & & 11 & 12 & 13 & 14 & 15 & 16 & 17 & 18 & & 20 & 21 & $\begin{array}{c}\text { Output } \\
\text { variable }\end{array}$ \\
\hline \multicolumn{20}{|c|}{ Remediation wells } & $\begin{array}{l}\text { Pumping } \\
\text { parameter }\end{array}$ & \\
\hline $\mathbf{0}$ & 0 & 1 & 0 & 1 & 0 & 1 & 0 & 0 & 1 & 1 & 1 & 1 & 1 & 0 & 0 & 0 & 1 & 1 & o & .50 & 1 \\
\hline $\mathbf{0}$ & 1 & 0 & $\mathbf{0}$ & 1 & 0 & 0 & 0 & 0 & 1 & 0 & 1 & 1 & 0 & 0 & 0 & 0 & 1 & 1 & $\mathbf{0}$ & .55 & 0 \\
\hline $\mathbf{0}$ & 0 & 0 & 0 & 1 & 1 & 0 & 0 & 0 & 0 & 0 & 0 & 0 & 1 & 1 & 0 & 1 & 0 & 1 & 0 & .65 & 0 \\
\hline
\end{tabular}

Figure 8. Twenty-well example of ANN groundwater remediation. (a) Possible locations of remediation wells. (b) Numerical mesh. 
during the time over which the aquifer is managed. Thus, the total pumping volume is equal to the length of the planning horizon multiplied by the summation of pumping rates in the different remediation wells.

The mathematical formulation of this problem is the same for the three-well example remediation formulation presented above in Eqs. (11) through (14) where $m$ is the length of the planning horizon, $j$ is the number of nodes that can be pumped as remediation wells, $q_{i}$ is the well pumping rate at location $i, c_{j}$ is the concentration of contaminant at monitor well $j, c_{\max }$ is the target concentration or action level at the monitor wells, and $q_{\max }$ is the maximum pumping capacity at the remediation wells.

The regulatory constraint, $c_{\max }$, is set at $30 \mathrm{ppb}$. The assumption is made that all wells have a maximum pumping capacity of $70 \mathrm{gpm}$, which is reasonable for deposits common to basin alluvial fill or glacial till. As this is not an exceptionally high yield relative to a sandstone or limestone aquifer, all wells will be operated at this maximum pumping capacity or not at all. In this discrete approach, injection or more levels of pumping could be considered. For example, instead of on at $70 \mathrm{gpm}$ or off, one might increase the number of possibilities to injection of 50 $\mathrm{gpm}$, off, pumping at $35 \mathrm{gpm}$, and pumping at $70 \mathrm{gpm}$. An injection scheme can be useful when complete hydraulic containment of the plume is necessary or when it is hoped that flushing the system will speed remediation. One drawback of injection is that regulatory agencies are reluctant to permit introduction of the most common source available for injection: contaminated water that has been pumped from the site and treated before injection.

The test remediation was implemented on a SUN Sparcstation I, and some of the flow and transport runs were performed on an ALLIANT FX/80. With twenty wells to be considered as possible pumping locations and wells being either on or off, this problem is a significant challenge from a combinatorial optimization perspective. Whereas the illustrative example above had $2^{3}$, or 8 , possible realizations of pumping, this example has $2^{20}$, or $1,048,576$ possible realizations. 


\subsubsection{Incorporation of the Management Formulation into the}

ANN Architecture

The type of ANN used is this study is a multilayer feed-forward perceptron trained with the use of backpropagation learning. It was not known at the beginning how many hidden layers would be used or how many hidden nodes would be needed in each hidden layer to achieve the best performance. The evolution of insight about improvements to the hidden-layer configuration is discussed below in the section "Training of the ANN." This section discusses the input and output.

The initial approach to incorporating the management formulation of the remediation problem into the ANN was to have the input vector represent a possible realization of pumping. As twenty possible pumping wells were being considered, each input was a row vector of twenty variables; a variable value of 1 indicated that the well was pumping at the maximum capacity (i.e., $70 \mathrm{gpm}$ ), and 0 indicated that the well was off.

The output was initially considered to be composed of two variables. The first represented whether or not the realization of pumping met the regulatory constraint, and the second represented the objective function to be minimized, i.e., the volume of water pumped. As each well had the same maximum capacity, the volume was a linear function of the number of pumping wells. Later it was recognized that this value could be calculated a priori, and it was therefore included as an input to the ANN to increase the information available to the network to aid its performance. The inclusion of this pumping parameter in the input vector did appear to increase generalization performance. Thus, the first 20 variables of the input vector represented the wells, with each variable being 1 or 0 , and the last variable represented the number of nonpumping wells normalized by the total number of wells (i.e., a linear combination of the first 20 variables). If no wells were pumping, this last input variable would be 1 , and if half of the wells were pumping, this last input would be 0.50 (Figure $8 \mathrm{~b}$ ). The one output variable represented whether the pumping realization was successful, i.e., if over the planning horizon, 
no concentration exceeded $30 \mathrm{ppb}$ in any of the monitor wells, the output was 1 , and if the realization was not successful, the output was 0 .

\subsubsection{Groundwater Flow and Transport Simulations (in Parallel) to Develop Training and Testing Patterns}

Four training and testing pattern sets of 50 pumping realizations were developed. The methods for generating these 4 sets were (1) patterns, (2) random-number generation, (3) variations on successful runs in the first two sets, and (4) hydrogeologic insight. The sets were not homogenized in order to find if certain methods for generating training sets had advantages over other methods. The first set was generated using simple patterns of on and off pumping such as $1010 \ldots, 110110 \ldots$, and $00010001 \ldots$. The second set was generated using a method of random-number generation [Press, 1986] that was reputed to have reduction of correlation between successive samples relative to most system-supplied random-number generators. The third set was generated with different pumping wells being added or deleted from examples of successful pumping realizations from the first two sets. A pumping realizations was defined as successful if the groundwater simulation implementing the realization did indeed keep contaminant mass from moving pass the monitor wells in concentrations above the regulatory contraints. The fourth set was generated by using hydrogeologic insight to choose likely realizations of pumping and variations thereof. Later it was found that increases in the number of hidden nodes improved network performance. The use of more hidden nodes increased the number of training patterns necessary, and a fifth input set of 45 was added. This fifth set was generated with variations of successful runs in the first four sets.

A 2-D hybrid finite-difference/finite-element flow and transport code SUTRA [Voss, 1984] was used to determine numerically whether or not the pumping realizations of the input sets met the regulatory constraints. The series of runs of the flow and transport code then determined the actual output value for the input patterns. Note that the hydraulic conductivity 
was two orders of magnitude higher in the arroyo sediments crossing the domain. The boundary conditions of the grid were no flow to the north and south and constant head to the east and west. The boundary conditions were set far enough away so as not to artificially limit pumping. Note that the arroyo is considered not to be a boundary, but rather a dry arroyo typical of basins receiving less than 15 inches of rainfall a year. The aquifer thickness was $80 \mathrm{~m}$ and the dispersivity was $50 \mathrm{~m}$ (Table 3 ).

Table 3. Aquifer and transport parameter values and model specifications.

\begin{tabular}{ll}
\hline \hline Aquifer parameter values: & \\
Base hydraulic conductivity & $6 \times 10^{-6} \mathrm{~m} / \mathrm{s}$ (range: \\
& silty sand-clean sand) \\
Porosity & 0.25 \\
Longitudinal dispersivity & $50 \mathrm{~m}$ \\
Transverse dispersivity & $12.5 \mathrm{~m}$ \\
Aquifer thickness & $100 \mathrm{~m}$ \\
Model specifications: & $15 \times 20$ \\
Grid dimensions & $100 \mathrm{~m} \times 100 \mathrm{~m}$ \\
Cell size & 336 \\
Number of grid nodes & Westward Gradient \\
Constant head boundaries at east and west & \\
No-flow boundaries at north and south & \\
\hline \hline
\end{tabular}

\subsubsection{Training of the ANN}

This section describes the evolution of the ANN and gives information on performance (1) once the 21 inputs and 1 output had been decided upon, and (2) once performance had been improved by increasing the number of hidden nodes from 2 to 3 to 4 . Remember that the number of hidden layers and the number of hidden layer nodes is a network parameter decided by the designer. An increase in hidden layer nodes increases the number of weights in the network. In general, increasing the number of weights increases the network's ability to describe the problem in more detail or "memorize". Reducing the number of weights forces the network to "generalize" more. Also increasing the number of hidden-layer units increases the weights and the necessary number of training patterns. This is often a motivation for keeping the 
network as simple as possible. Each application must find an appropriate architecture (i.e., suitable middle ground between generalization and memorization capacity) for the network to adequately solve the problem. Generalization capacity is discussed in more detail in Section 4.0.

\subsubsection{Measure of Success or Performance}

Two common strategies for measuring how successfully the ANN has been trained are to test the capability of the network to predict correctly the output for (1) the input sets that were originally used to train the network, and (2) input sets that were not in the training set. The two strategies above are somewhat analogous to the calibration (strategy 1) and verification (strategy 2) processes that are used to insure the reliability of models or experimental devices. The first strategy is often referred to as an accuracy performance, and the second as a generalization performance.

\subsubsection{Training Rounds}

During the first round of training (Table 4), the original four pattern sets were grouped into series of two, each with 100 training patterns and 100 test patterns. One hidden layer with four hidden nodes was used. This pattern is indicated along with the 21 inputs and 1 output in the architecture abbreviation of 21-4-1. The accuracy performance was $100 \%$ for each input series; the generalization performance ranged from 82 to $91 \%$, with the highest generalization performance resulting from training with the 2,4 series and 3,4 series. The trained networks were tested for generalization performance on the sets which were not involved in their training; for example, the network trained on sets 2 and 4 would be tested on sets 1 and 3 .

During the second round of training (Table 4), the original four pattern sets were grouped into series of three sets for training (150 training patterns) and one set for testing (50 test patterns). Note that each training of the network is independent of another. The architecture was again $21-4-1$. The accuracy performance was $100 \%$ for all of the series except $2,3,4$, which did not converge as noted by an asterisk. The large number of iterations appeared to improve 
the generalization performance of this series, as it was the highest generalization performance of this training round at $92 \%$. The lowest generalizaiton performance of this training round was $52 \%$ for series $1,2,3$. Note that the $2,3,4$ series has all the input sets of the top performing series in training round 1 (i.e., 2,4 and 3,4 ). This may suggest the strength of the latter three methods for training pattern development, i.e., random generation, variation of successful patterns, and hydrogeologic insight. 
Table 4. Accuracy and generalization performances for seven ANN training rounds.

\begin{tabular}{|c|c|c|c|c|c|c|}
\hline Architecture & \multicolumn{2}{|c|}{$\begin{array}{l}\text { Sets in Input } \\
\text { Series (No. } \\
\text { of Patterns) }\end{array}$} & \multirow[t]{2}{*}{ Accuracy } & \multirow[t]{2}{*}{ Generalization } & $\begin{array}{l}\text { Number } \\
\text { of Weights }\end{array}$ & \multirow[t]{2}{*}{$\begin{array}{l}\text { Number of } \\
\text { Function } \\
\text { Evaluations@ }\end{array}$} \\
\hline Training row & & & & & & \\
\hline $21-4-1$ & 1,2 & (100) & $100 \%$ & $82 \%$ & 93 & 119 \\
\hline $21-4-1$ & 1,3 & (100) & $100 \%$ & $82 \%$ & 93 & 126 \\
\hline $21-4-1$ & 1,4 & (100) & $100 \%$ & $85 \%$ & 93 & 118 \\
\hline $21-4-1$ & 2,3 & $(100)$ & $100 \%$ & $91 \%$ & 93 & 135 \\
\hline $21-4-1$ & 2,4 & (100) & $100 \%$ & $91 \%$ & 93 & 228 \\
\hline 21-4-1 & 3,4 & (100) & $100 \%$ & $69 \%$ & 93 & 158 \\
\hline \multicolumn{7}{|c|}{ Training round 2} \\
\hline $21-4-1$ & $1,2,3$ & (150) & $100 \%$ & $52 \%$ & 93 & - \\
\hline 21-4-1 & $2,3,4$ & (150) & $92.7 \%$ & $92 \%$ & 93 & $4560^{*}$ \\
\hline $21-4-1$ & $1,3,4$ & (150) & $100 \%$ & $72 \%$ & 93 & 172 \\
\hline $21-4-1$ & $1,2,4$ & (150) & $100 \%$ & $86 \%$ & 93 & 1295 \\
\hline \multicolumn{7}{|c|}{ Training round 3} \\
\hline $21-5-1$ & $1,2,4$ & (150) & $100 \%$ & $94 \%$ & 116 & 301 \\
\hline $21-6-1$ & $1,2,4$ & (150) & $100 \%$ & $100 \%$ & 139 & 3571 \\
\hline $21-2-2-1$ & $1,2,4$ & (150) & $98 \%$ & $72 \%$ & 53 & $5000^{*}$ \\
\hline $21-4-1$ & $1,2,4$ & (150) & $100 \%$ & $86 \%$ & 93 & 1295 \\
\hline \multicolumn{7}{|c|}{ Training round 4} \\
\hline $21-6-1$ & $1,2,3$ & (150) & $100 \%$ & $72 \%$ & 139 & 68 \\
\hline $21-6-1$ & $2,3,4$ & (150) & $100 \%$ & $86 \%$ & 139 & 477 \\
\hline $21-6-1$ & $1,3,4$ & (150) & $100 \%$ & $\mathbf{8 2} \%$ & 139 & 205 \\
\hline $21-6-1$ & $1,2,4$ & (150) & $100 \%$ & $100 \%$ & 139 & 3571 \\
\hline \multicolumn{7}{|c|}{ Training round 5} \\
\hline $21-7-1$ & $1,2,3$ & (150) & $100 \%$ & $62 \%$ & 162 & 148 \\
\hline $21-7-1$ & $2,3,4$ & (150) & $100 \%$ & $92 \%$ & 162 & 353 \\
\hline $21-7-1$ & $1,3,4$ & (150) & $100 \%$ & $80 \%$ & 162 & 257 \\
\hline $21-7-1$ & $1,2,4$ & (150) & $99.3 \%$ & $98 \%$ & 162 & $1727^{*}$ \\
\hline \multicolumn{7}{|c|}{ Training round 6} \\
\hline $21-7-1$ & $1,2,3$, & 5 (195) & $99.5 \%$ & $80 \%$ & 162 & $1583^{*}$ \\
\hline 21-7-1 & $2,3,4$ & $5(195)$ & $100 \%$ & $90 \%$ & 162 & 1300 \\
\hline $21-7-1$ & $1,3,4$, & 5 (195) & $100 \%$ & $82 \%$ & 162 & 238 \\
\hline $21-7-1$ & $1,2,4$, & 5 (195) & $99 \%$ & $96 \%$ & 162 & $2883^{*}$ \\
\hline \multicolumn{7}{|c|}{ Training round 7 (weight elim.) } \\
\hline $21-7-1$ & $1,2,3$, & 5 (195) & $87.7 \%$ & $94 \%$ & 162 & $1265^{*}$ \\
\hline $21-7-1$ & $2,3,4$ & 5 (195) & $85.1 \%$ & $98 \%$ & 162 & - \\
\hline $21-7-1$ & $1,3,4$, & 5 (195) & $85.6 \%$ & $96 \%$ & 162 & - \\
\hline $21-7-1$ & $1,2,4$, & 5 (195) & $89.7 \%$ & $92 \%$ & 162 & $1189^{*}$ \\
\hline
\end{tabular}

Failed to reach a successful optimization.

(1) Number of evaluations the error function required to reach a final value of $<10^{-5}$. 
The third round of training involved variation of hidden-layer architecture using one training series, $1,2,4$, which had a medium generalization performance of $86 \%$ in the second training round The series $1,2,4$ training series was chosen over the top performing series $2,3,4$ of round 2 because the latter's associated test set was judged to be relatively easy with less variation. Thus, the test set associated with training series $1,2,4$ was thought to be a more robust test. The number of hidden nodes in a one-hidden-layer architecture was increased from 4 to 5 to 6 , resulting in increases in generalization performance from $86 \%$ to $94 \%$ to $100 \%$. The architecture of two hidden layers each with two hidden-layer nodes was also tried (21-2-2-1); however, the generalization performance was only $72 \%$. Also, the accuracy performance of this architecture was $98 \%$, as the training had difficulty in convergence. This training round emphasized the importance of increasing the number of hidden-layer nodes to an appropriate number for the network to be more capable of mapping the structure of the nonlinearity of this remediation problem in the weights. This issue of the appropriate number of hidden-layer nodes for a network will be discussed more in Section 6.0.

Armed with this knowledge of increasing generalization performance with more hidden nodes, the fourth round of training increased the number of hidden nodes to six for all four series of three sets for training (150 training patterns) and one set for testing ( 50 test patterns). Performance was increased to between $72 \%$ and $100 \%$ (compare with $52 \%$ and $92 \%$ for training round 2).

The fifth round of training sought further improvement by increasing the number of hidden units to 7. However, overall generalization performance fell off: compare the range of $72 \%$ to $100 \%$ with $62 \%$ to $98 \%$. This range is believed to be the result of there being more weights in the 21-7-1 architecture (160) than the number of training patterns (150).

An additional 45 training examples were included in the sixth training round, which increased generalization performance to between $80 \%$ and $96 \%$. Note that there was some difficulty in training convergence for series $1,2,3,5$ and $1,2,4,5$. This difficulty may be a result of additional noise in pattern set 5. Or perhaps more likely is the difficulty of differentiating 
between the narrow variations between set 5 patterns; remember pattern set 5 consisted of variations on successful patterns. Adding more training patterns may possibly increase the noise or idiosyncracies of the training set. The seventh training round will be discussed in the next section.

\subsubsection{Weight Elimination to Improve Network Generalization Performance}

As mentioned above, there is the possibility of overtraining in some networks. In this situation the network continues to minimize the number of errors between desired and predicted outputs, i.e., accuracy performance, at the expense of the generalization performance. In other words, the ANN can be improving the error of the training patterns, but training on noise in the training set. Despite additional iterations and improved network accuracy performance, the network capability to predict patterns it has not seen actually decreases.

To explore the possibility that this was occurring with the ANN of this example, the network was trained with the weight-elimination method of Weigend et al. [1991a], implemented by Jang et al. [1991]. The definition of system error, E, that was used was the same as that of Jang et al. [1991]:

$E=\frac{\text { errpot }}{J}$

where

errpat $=\underset{i=1}{J}(\text { target }- \text { output }\}_{i}^{2}$

Training with the four sets of 195 training patterns and 50 testing patterns revealed overtraining to be a factor in this application (Figure 9). For each testing series there is a noticeable point of training, somewhere between 175 and 375 iterations, at which testing error 
increases despite the decrease in training error. The breakpoint in testing error occurs for the four testing series (a) $1,2,3,5$, (b) $2,3,4,5$, (c) $1,3,4,5$, and (d) $1,2,4,5$ at iteration number 375,350 , 225 , and 175, respectively. The generalization performance does improve for three of these four sets when the weight elimination procedures are implemented (Table 4). Note also a small decrease in accuracy of prediction of the test patterns themselves. This indicates that as designed, the weight elimination procedures inhibit memorization of the test patterns in order to increase the network's ability to generalize its understanding of new patterns.

\subsubsection{Success Prediction of Combinatorial Management Possibilities}

Once the network has been trained to an acceptable level of performance, it can be used to examine as many combinatorial possibilities as desired. Theoretically, for this example, all $1,048,576$ possibilities could be examined to ensure a global minimum. As this is infeasible, selective search algorithms were considered. The original selection of patterns involved random patterns, as well as patterns based on successful patterns that removed one then another of the pumping wells. This was fairly successful in this example problem because the objective of minimizing pumping wells stood to decrease as wells were removed. This approach is not necessarily as successful where the objective function does not have this monotonic structure. For example, in a more recent field-scale application (Section 5.1) the objective function was to maximize the mass output; taking a well away may help or hurt this objective. Thus, more robust search procedures were examined, and the GA was selected as well-suited for the task of pattern generation.

For this example, the process was stopped after 2000 possible patterns were examined, and the seven highest-ranking realizations were selected (Fig $10 \mathrm{a}-\mathrm{g}$ ). The first 1000 possible 


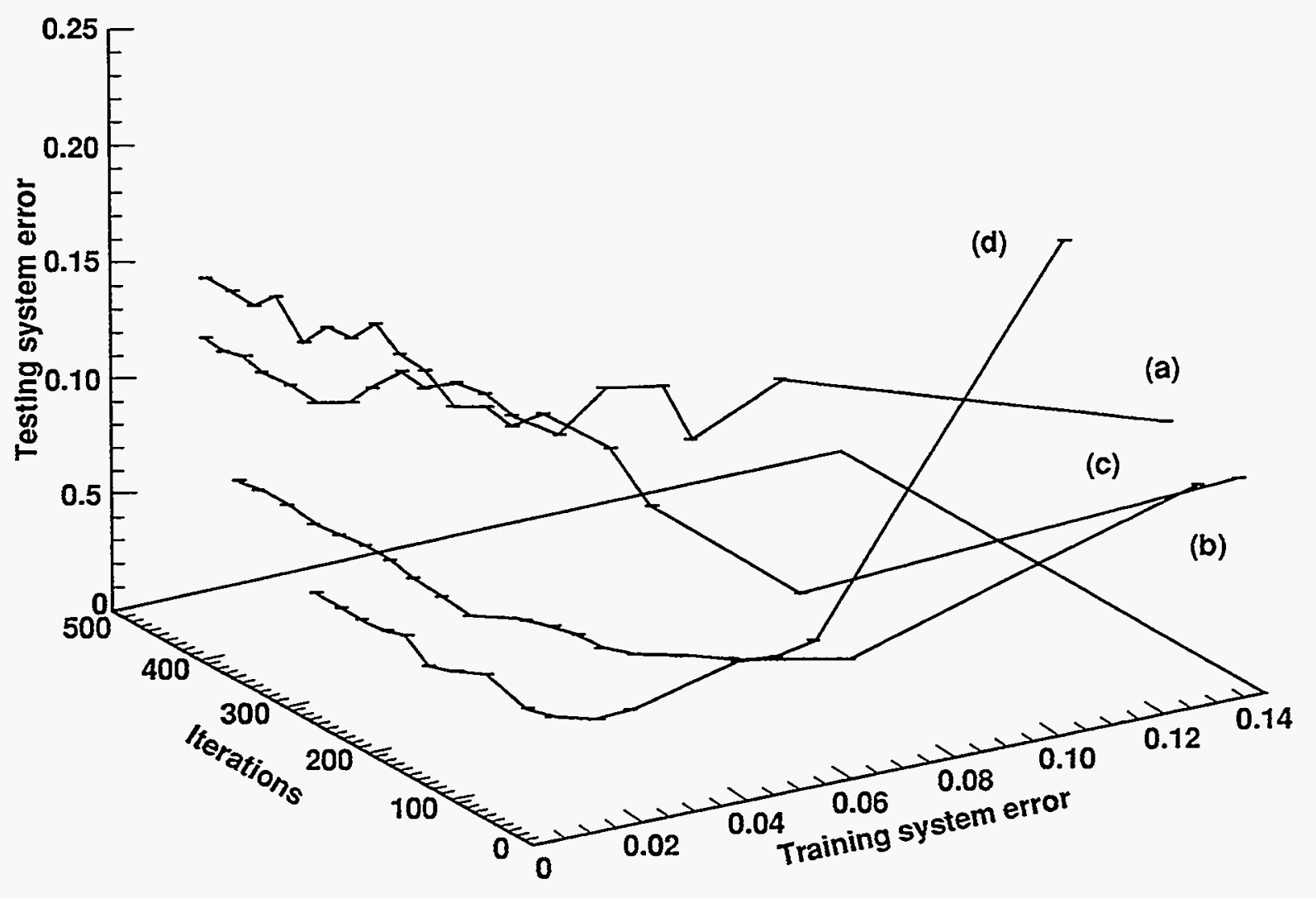

Figure 9. $X Y Z$ plot of training sytem error, versus number of iterations, versus testing system error in weight elimination training for input series (a) $1,2,3,5$, (b) $2,3,4,5$, (c) $1,3,4,5$, and (d) $1,2,4,5$. 
(a)

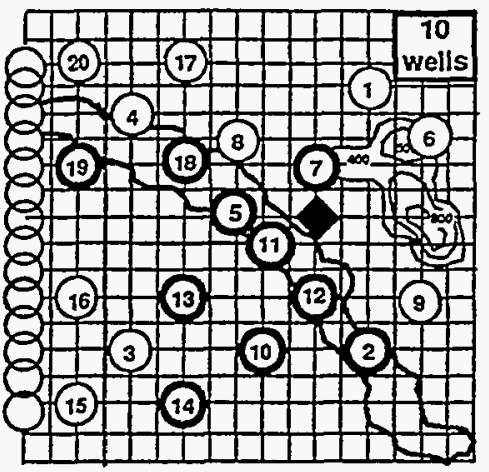

123345678891071127314157617181820

(c)

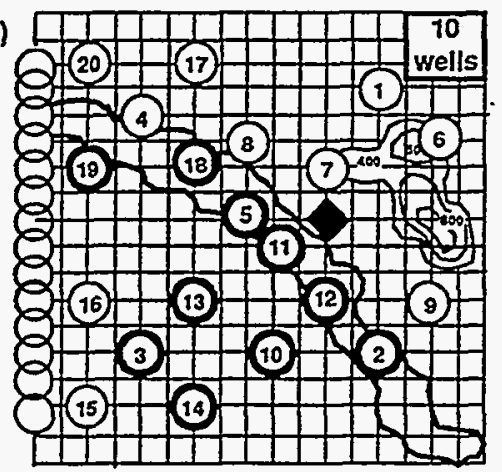

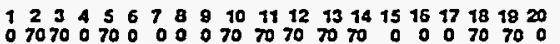

(e)

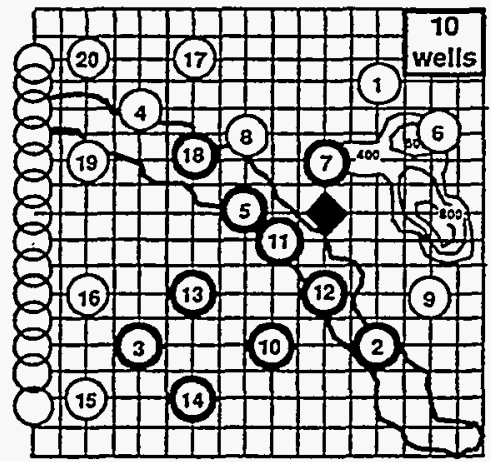

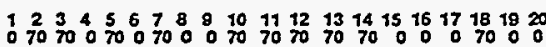

(g)

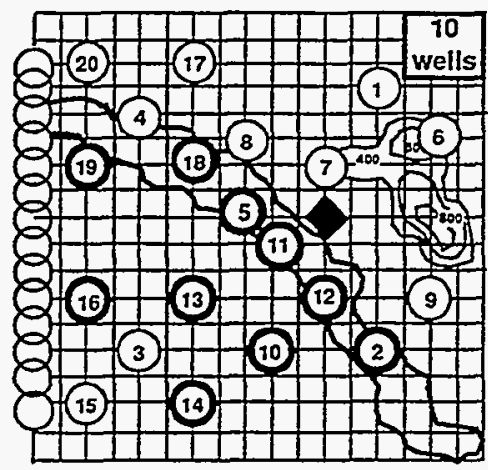

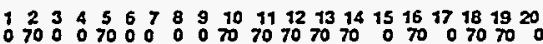

(b)

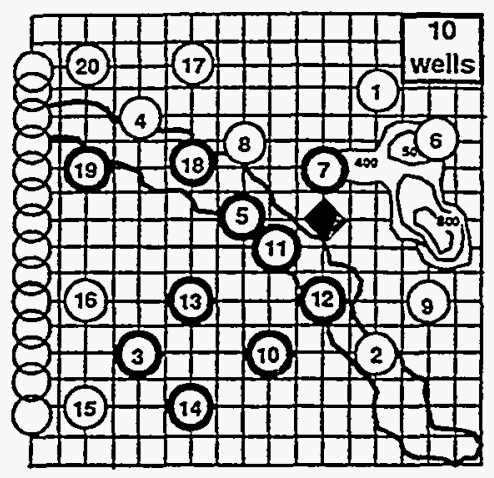

Wellno:

(d)

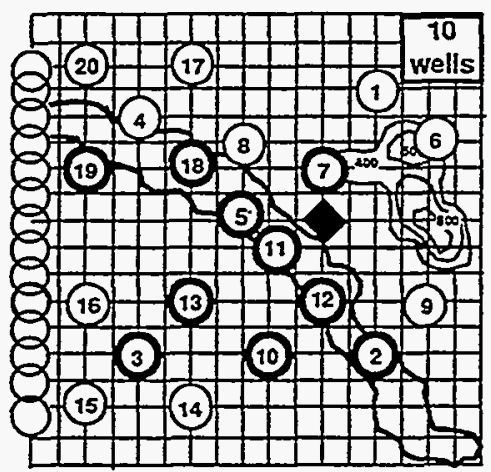

Pull noi

(f)

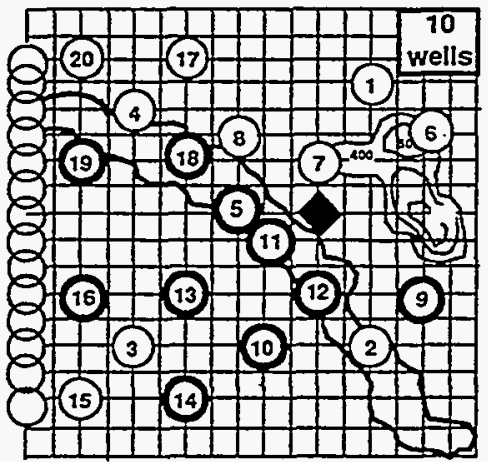

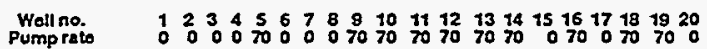

(h)

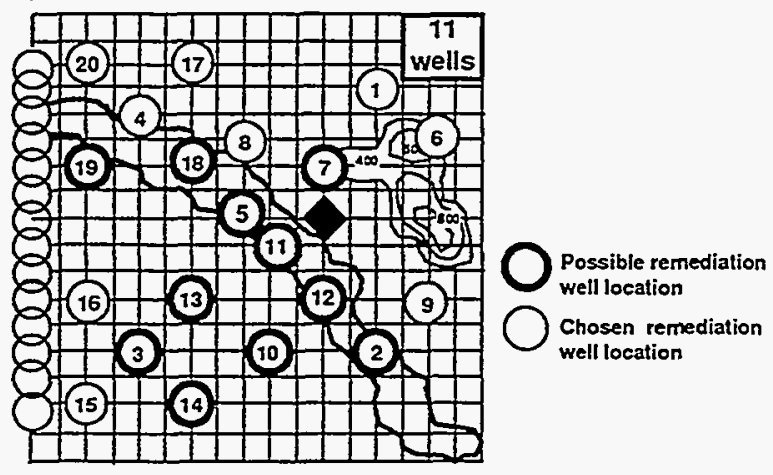

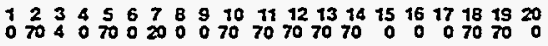

Figure 10. Seven recommended management solutions from the ANN groundwater management model [(a) through (g)], compared with a recommended solution from a nonlinear programming management (NLP) model (h). 
pumping patterns were selected randomly as well as by removing wells from successful pumping patterns. This method was augmented with the genetic algorithm. An initial population of 20 successful patterns was evolved through 50 generations according to a simple three-operator GA to produce 1000 pumping realizations. Each of the highest-ranked realizations employed 10 pumping wells. These recommended management solutions consistently suggested the use of well Nos. $5,10,11,12,13$, and 18. The other wells used to meet regulatory constraints were Nos. $2,3,7,9,14,16$, and 19. The highest-ranked realizations never used wells $1,4,6,8,15,17$, or 20 .

\subsubsection{Confirmation of Management Solutions}

The recommended ANN pumping realizations were confirmed as successful with the aid of the flow and transport code. Surface contour plots over time (Figure 11) show unremediated contaminant concentrations approaching $120 \mathrm{ppb}$. Similar plots produced with the implementation of ANN-recommended management solutions showed no concentrations above $30 \mathrm{ppb}$ reaching the monitor wells (Figures 12 and 13) over a management period of 50 years.

\subsubsection{Comparison with a More Conventional Technique}

A more conventional groundwater management model using nonlinear programming was compared with the ANN groundwater management solutions. The two primary components of the nonlinear programming management model are (1) the nonlinear optimization model NPSOL [Gill et al., 1986], and (2) the same groundwater simulation model used by the ANN management model SUTRA [Voss, 1984].

The optimization model handles the input of objective functions, water quality constraints, and hydraulic constraints. The groundwater simulation model, linked as an independent submodel or subroutine, controls the input of hydrogeologic data, the domain of interest, flow velocities, and contaminant concentrations. The optimization model then calls the 

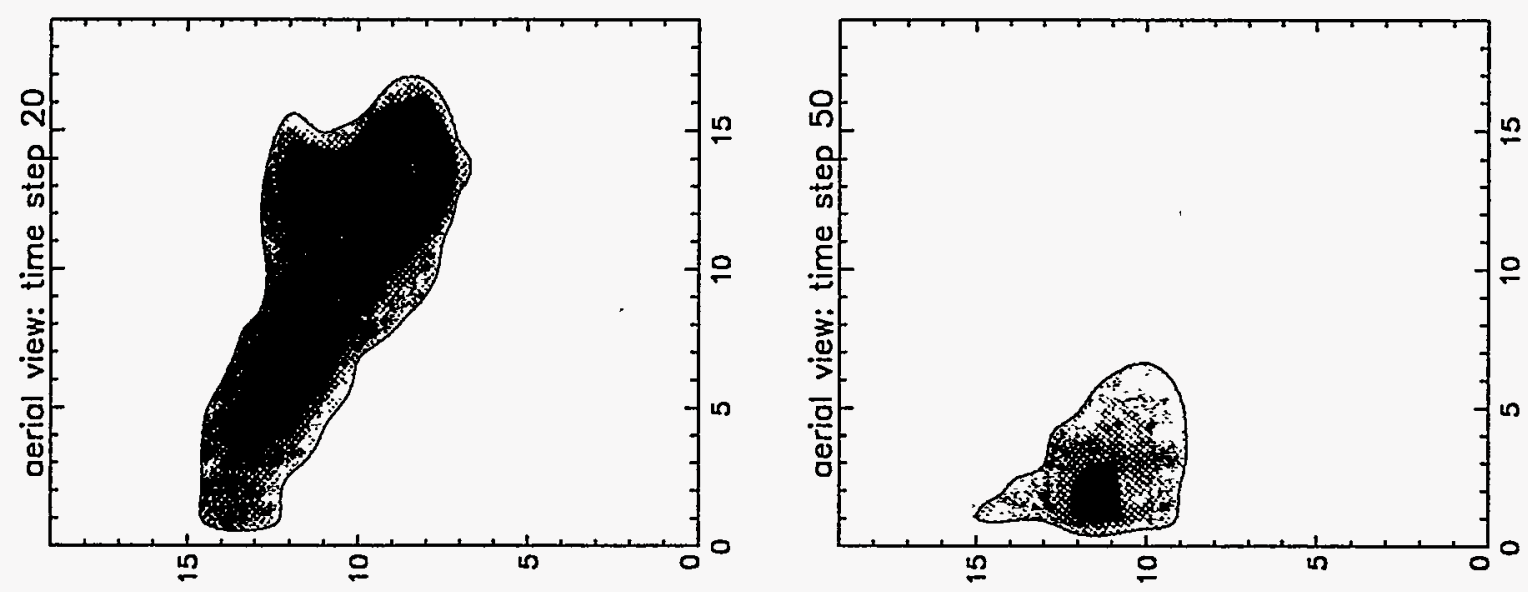

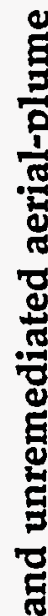
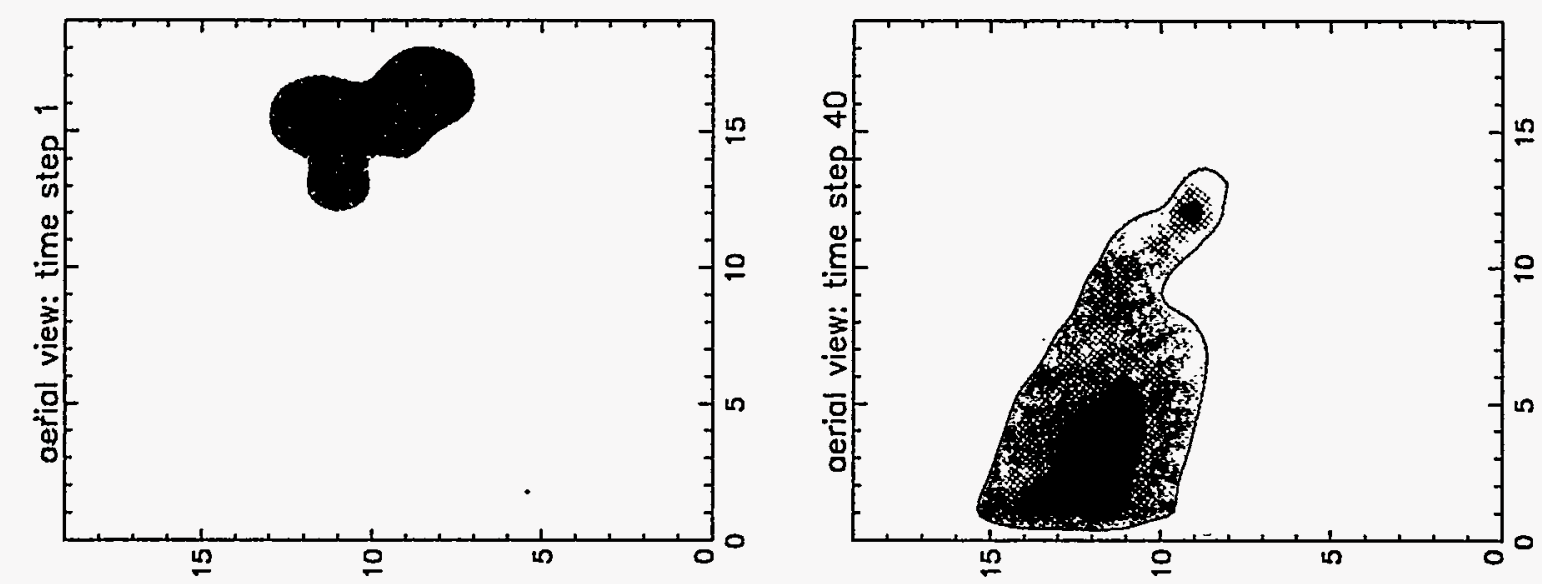

\begin{tabular}{l}
0 \\
0 \\
+0 \\
0 \\
0 \\
$\frac{1}{0}$ \\
$\frac{1}{5}$ \\
\hline
\end{tabular}

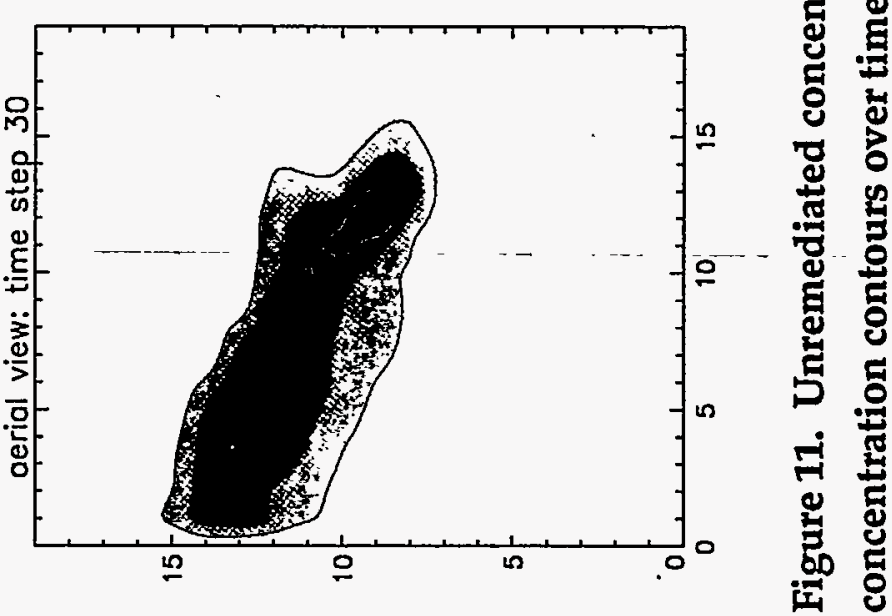

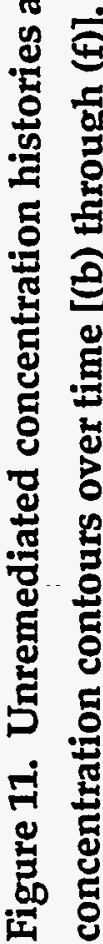



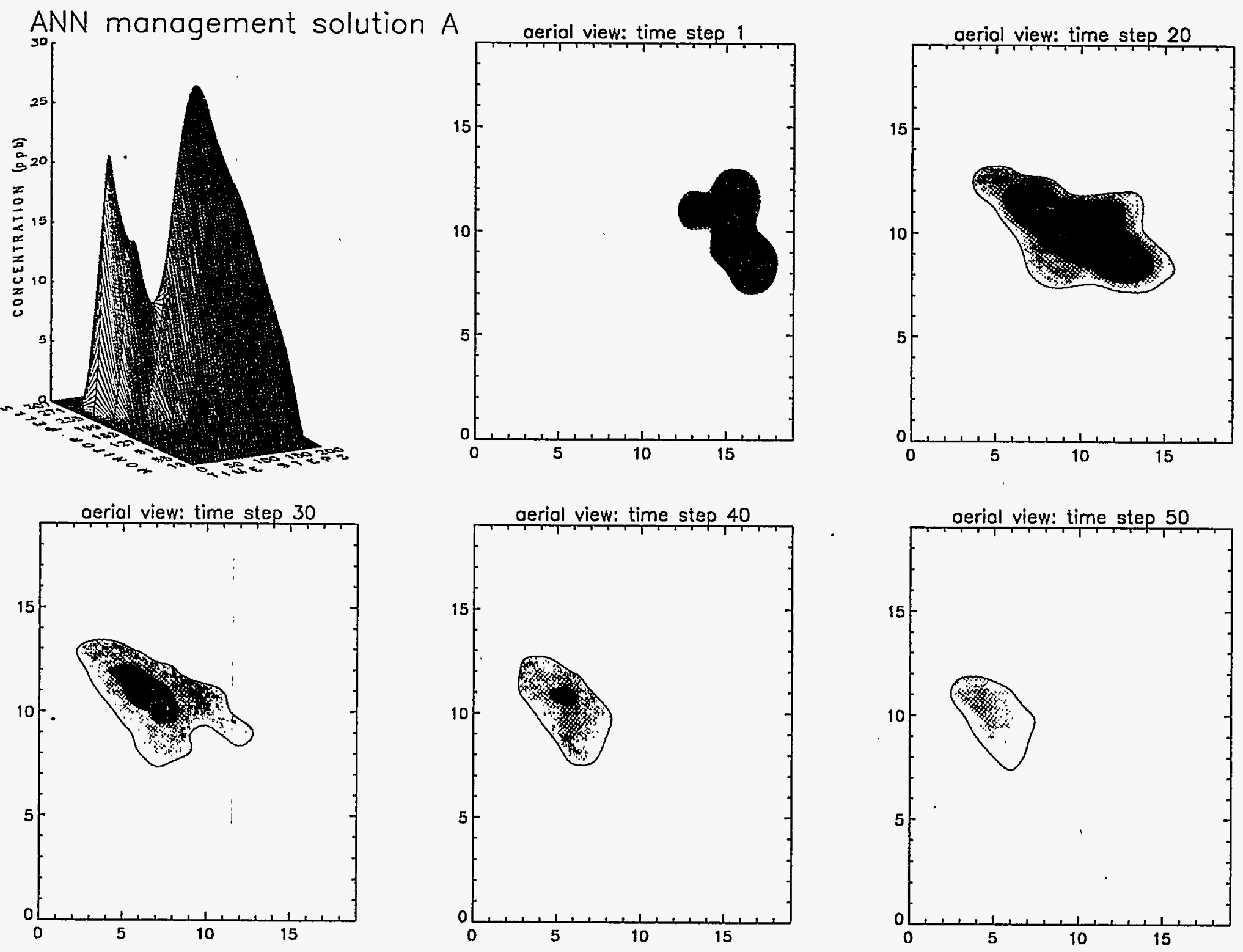

Figure 12. Successful ANN management solution A: Concentration histories at the monitor wells (a), and aerial-plume concentration contours over time [(b) through (f)]. 

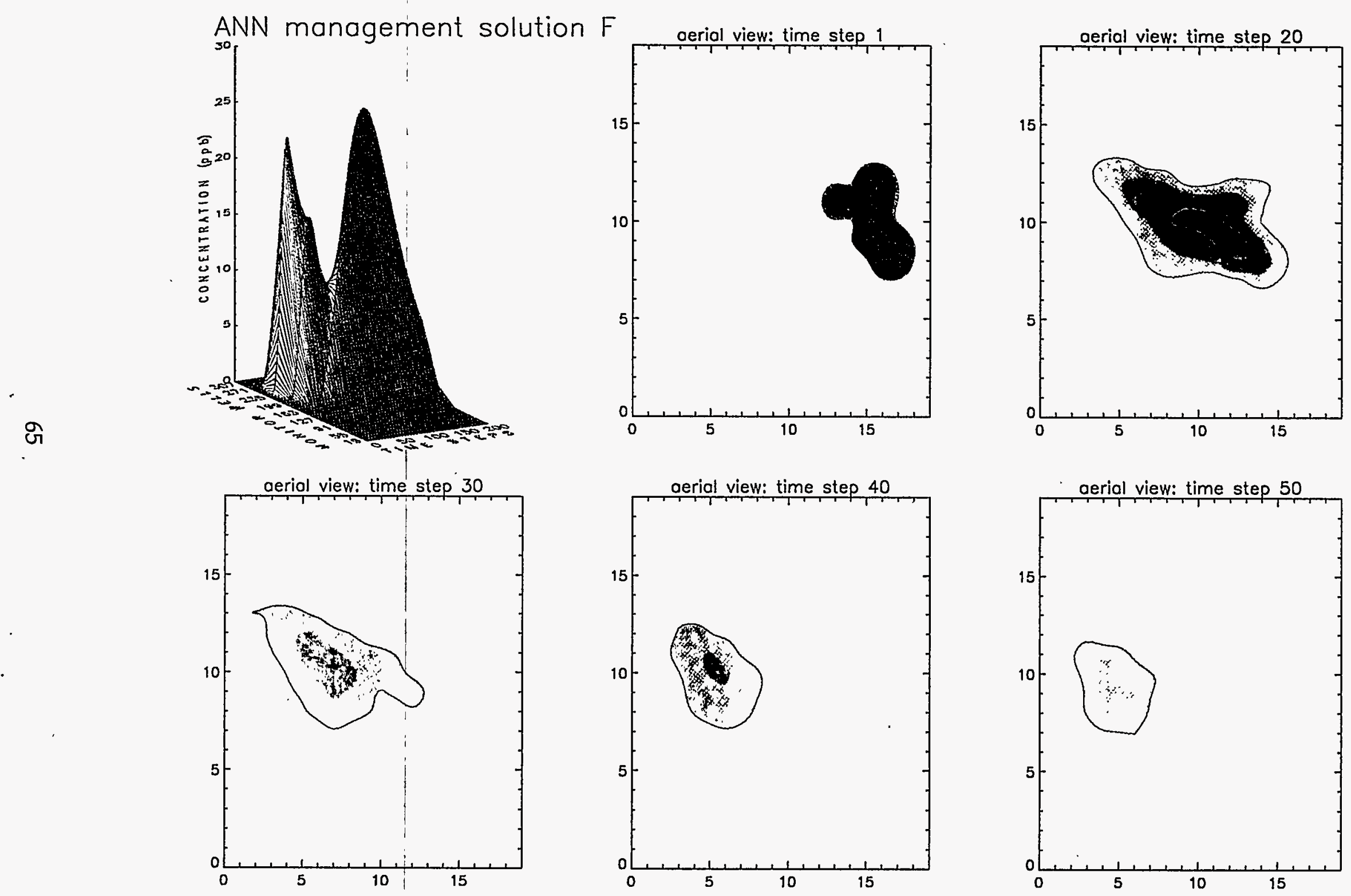

Figure 13. Successful ANN management solution F: Concentration histories at the monitor wells (a), and aerial-plume concentration contours over time [(b) through (f)]. 
groundwater simulation submodel to calculate the state variables or flow regime and the distribution of contaminants caused by the initial pumping rates. The optimization model will vary these pumping rates by a small increment and call the groundwater simulation submodel again to see the resulting change in contamination distribution. After several of these calls to the groundwater simulation submodel, the optimization model will be able to determine how the concentrations change with respect to the changes in pumping rates in the different wells. This process is the evaluation of the Jacobian matrix by perturbations in the pumping rates. From the Jacobian, the optimization model can decide whether to increase or decrease the level of pumping in a particular well. The entire process will continue until the optimization model's conditions for optimality and convergence are reached or until the optimization model decides it cannot obtain any improvement in the solution. Note that this approach requires that the runs of the groundwater simulation code (i.e., for flow and transport) be performed sequentially and that appropriate arrays, such as concentrations at the monitor wells, pumping rates at the remediation wells, etc., be passed between the optimization code and the groundwater simulation code.

The nonlinear programming management model recommended a solution (Figure 10h) that was very similar to the ANN management solution (A) (Figure 10a). The solution suggests 9 wells pumping at their full capacity of $70 \mathrm{gpm}$ with an additional two wells pumping at a partial capacity (well No. 3:4 gpm, and well No. 7:20 gpm). An engineering approach to implementing this solution would in all likelihood either not put in well No. 3 or put it in pumping at a volume closer to full capacity; it would not be efficient to construct a well for a 4-gpm yield. If we disregard well No. 3 pumping, the nonlinear programming solution uses the same 10 wells as the ANN solution (A), only with a total pumping rate which is approximately $50 \mathrm{gpm}$ less. The nonlinear programming management model called the flow and transport code 206 times to arrive at this solution. Consider that 7 similar management solutions were recommended by the ANN trained on results from 195 calls to the flow and transport code. Keep in mind that for any particular problem the number of calls to the flow and transport code may vary. Also the 
nonlinear programming management model spends a fair amount of its calls trying to define pumping rates. The important issue here is that when the flow and transport simulations are complex the ANN approach's ability to perform them in parallel is an advantage over the sequential NLP approach. For example, even in this relatively simple example the flow code took approximately 1 hour on a SPARC 1 workstation. The flow and transport simulations upon which a NLP approach would base a management solution would require approximately 200 real time hours. The transport simulations upon which an ANN approach would base a management solution could be accomplished in 200 real time hours if only one workstation were available, but could be accomplished in only 1 real time hour if 200 workstations were available. The important issue here is parallel possibilities in the design structure of the management approach.

\subsubsection{Multiple Objectives}

Having three equally weighted objectives will be discussed in a linking of three ANNs in Section 5.0. However, for the sake of exploring a simple way to narrow the choice between the 7 suggested ANN solutions, this section explores how additional objectives can be examined. Two objectives that can be incorporated in a straightforward manner, mass removal and cost of piping to surface-treatment facilities, are briefly demonstrated here

The mass removed during each remediation was calculated with a cumulative mass balance. There was some deviation of mass removed, with ANN management solution F removing the most mass (Table 5). Note that the contamination is really two converging plumes, with the highest concentration to the south. Solution $\mathrm{F}$ is the only one to turn on well No.9, which is closest to the southern hot spot. 
Table 5. Multiple objectives for the twenty-well test remediation.

\begin{tabular}{llllllll}
\hline $\begin{array}{l}\text { ANN } \\
\text { Remedial } \\
\text { Solution }\end{array}$ & $\begin{array}{l}\text { Number of Total } \\
\text { Wells } \\
\text { Pumping }\end{array}$ & $\begin{array}{l}\text { Surface } \\
\text { Distance to (Dollars) } \\
\text { Treatment }\end{array}$ & $\begin{array}{l}\text { Cost per ft } \\
\text { of Pipe }\end{array}$ & $\begin{array}{l}\text { Total Cost } \\
\text { of Surface } \\
\text { Piping } \\
\text { (Dollars) }\end{array}$ & $\begin{array}{l}\text { Mass } \\
\text { Removed } \\
\left(\text { ( }^{*} 10 \text { e10) }\right.\end{array}$ & $\begin{array}{l}\text { Rank by } \\
\text { Maximum } \\
\text { Mass } \\
\text { Removal }\end{array}$ & $\begin{array}{l}\text { Rank by } \\
\text { Minimum } \\
\text { Cost of } \\
\text { Surface } \\
\text { Piping }\end{array}$ \\
A & 10 & 19,780 & 70 & 441,000 & 1.597 & 3 & 1 \\
B & 10 & 21,350 & 70 & 476,000 & 1.546 & 6 & 3 \\
C & 10 & 22,920 & 70 & 511,000 & 1.619 & 2 & 4 \\
D & 10 & 19,780 & 70 & 441,000 & 1.561 & 4 & 1 \\
E & 10 & 20,090 & 70 & 448,000 & 1.479 & 7 & 2 \\
F & 10 & 22,920 & 70 & 511,000 & 1.804 & 1 & 4 \\
G & 10 & 22,920 & 70 & 511,000 & 1.559 & 5 & 4 \\
\hline \hline
\end{tabular}

One can consider the additional cost of that a pump-and-treat remediation has for pipe between the remediation wells and the treatment facility. The cost was calculated for the seven ANN management solutions with a treatment facility located close to the bulk of the wells (Figure 8 ) and a cost of about $\$ 70 / \mathrm{ft}$ for piping (Table 5). This value was a median cost based on calculations performed at Lawrence Livermore National Laboratory [Dresen et al., 1991], which suggested a range between $\$ 40 / \mathrm{ft}$ for surface piping and $\$ 100 / \mathrm{ft}$ for double-contained subsurface piping. These estimates include the cost of materials, design, and installation. The management solutions were then ranked between 1 and 4 , with rank 1 being the solution which also minimized the cost of piping. Some of the management solutions had the same cost and were therefore ranked the same. A savings of just under $\$ 220,000$ would have resulted from implementation of the highest-ranked management solution over implementation of the lowestranked solution.

\subsubsection{Discussion of Twenty-well Remediation Problem}

Two aspects of the ANN implementation can be readily seen to impact the performance of the network: (1) the grouping of training and testing patterns, and (2) the number of hidden units. 
Common sense would dictate that the similarity of testing to training patterns would increase generalization performance, i.e., success of the ANN, which learned from the training patterns to predict the unseen test patterns. One might thus theorize that homogenization of the original pattern sets would have yielded better performance. However, the original patterns were grouped in series of training and testing patterns rather than homogenized to ascertain whether certain approaches to pattern generation yielded greater generalization performance. The preservation of generation identity was neither an exhaustive nor perfect statistical analysis; however, interesting trends can be observed.

Early in the training process, with only 100 patterns used in training and 100 patterns used in testing, the most successful combinations in training round 1 (Table 4) were the randomly generated patterns (set 2) and one of either set 3 or set 4 (i.e., patterns generated from hydrologic insight and variations on successful patterns in the previous sets, respectively). The relatively higher generalization scores of these sets continued, with the highest performance in round 2 being series $2,3,4$ and the second highest series $1,2,4$. The highest generalization performances in rounds 4 and 6 were series $1,2,4$ and 1,2,4,5, respectively. The second highest scores went to series $2,3,4$ and $2,3,4,5$, respectively. These results were interpreted as suggesting that the random generation of patterns was helpful in covering the search space, and the generation of patterns by hydrologic insight provided "good" examples that increased the network's capability to discern which of the wells had the greatest impact on outcome of remedial success.

The progression of overall generalization performance was improved by the addition of more hidden units. A rule of thumb is that the more complex nonlinear problems will need more hidden units. See Section 6.0 for more discussion of empirical and theoretical background. Note that the illustrative example was able to capture the behavior of the nonlinear system (i.e., attain good performance) with only two hidden nodes. Yet, the more complex twenty-well test remediation required more hidden nodes to achieve adequate generalization capabilities. For certain individual training/testing combinations, the performance dropped off as the number of 
hidden units increased. Comparing training rounds 4 and 6 , the generalization performance improved or stayed the same for the first three series, yet fell off for the fourth series. This lowering of performance could be due to the addition of the "noise" or complexity in the 45 extra examples, for while the extra examples and another hidden unit between rounds 4 and 6 helped the first three series, it hurt the last. The difficulty observed with this last series with regard to generalization performance could also be due to difficulty in reaching a successful optimization. However, note that both the first and last series had difficulty in reaching a successful optimization, with accuracy performance being less than $100 \%$. The fourth series was also different in its response to implementation of the weight elimination procedures. The first three series improved significantly in generalization performance with the weight elimination of round 7. However the fourth series actually lost $4 \%$ in generalization performance. Note that the accuracy performance decreased in training round 7 as expected because the network is encouraged not to overtrain, or focus too much on memorizing the training set in hopes of improving the generalization performance. Even with the $4 \%$ decrease in generalization performance of series 4 , the final overall generalization performance of all series is quite good, being between $92 \%$ and $98 \%$. 


\subsection{Network Dissection}

The way a backpropagation network maps the input patterns to a set of output patterns does not involve making any assumptions about the form of the patterns. The network "learns" the patterns by example and organizes its hidden layers into a system of feature detectors necessary to classify the problem. The weights of the network, originally set to small random numbers, are varied according to the backpropagation algorithm so that the final set of network weights can be summed and transformed with the input patterns to determine most correctly the outputs of the training patterns. Dissecting the network to discover the structure of these feature detectors is approached by examining the network weights. The summed weights at each hidden node become a ranking of importance of the inputs in order to reflect the salient features of the training patterns.

Analysis of the network weights is a very problematic proposition in ANN due to the nonlinear transforms the weighted sums are passed through. Also, there is no guarantee that the hidden nodes will become the same collection of feature detectors from training with one set of patterns to training with another set of patterns. Yet, the formulation chosen for this groundwater management problem should have a greater chance of success, as inputs relate specifically to a spatial well location. Greater understanding of the evolution and statistical significance of the weights will contribute to both the field of neural network technology and the field of solute transport modeling. In the case of solute transport modeling, understanding of the weights will more directly correlate how the network has mapped the physics of solute transport. This should make the decisions more accessible to hydroscientists and less of a "black box". Greater insight should result concerning the nonlinearity of the well interference patterns and competition for contaminants. In the case of neural network technology, the dissection of 
the weights will clarify how the network learns and organizes its decisions, which is an ongoing research interest within the field of neural networks.

\subsection{Simple Example of Weight Maturation}

Let us return to the three-well example remediation discussed in section 3.1.1. Recall that in this simple example the network had to learn that well number 2 being turned on (input set to 1) was the dominant factor in the defined success of remediation. The weights between the nodes of the network will be observed as they mature during learning of successive training pumping realizations and output patterns. The weight maturation will be correlated to the increase in the network's ability to correctly predict outcome of the remaining possible pumping patterns.

Let us refer back to Figure 3 which outlines all eight possible pumping realizations and their correct output. We will go through 4 training sequences with the number of training patterns being 1 for training sequence 1,2 for training sequence 2, 3 for training sequence 3 , and 4 for training sequence 4 (Figure 14). Thus, there is an increase of one training pattern between each sequence. The architecture will be as simple as possible, three input nodes, one hidden layer node, and one output node. Note that this architecture has one less hidden layer node than the network for which results were reported in Table 2. The use of the most simple architecture will make it easier to observe the weight maturation. Thus six weights are associated with the network, three between the inputs and the hidden layer node, one bias weight for the hidden node, one between the hidden node and the output node, and one bias weight for the output node. The bias weight refers here to a value associated with each node of the hidden and output layers. This bias weight is multiplied by a constant value, usually 1 , and acts like an extra signal coming in from the layer below The purpose of the bias weight is to make training easier by helping to appropriately scale the threshold function.

For the first training sequence the network was only given one pattern (1 $11: 1)$ to learn. In this notation the first three values before the colon are input values, and the last value after the 

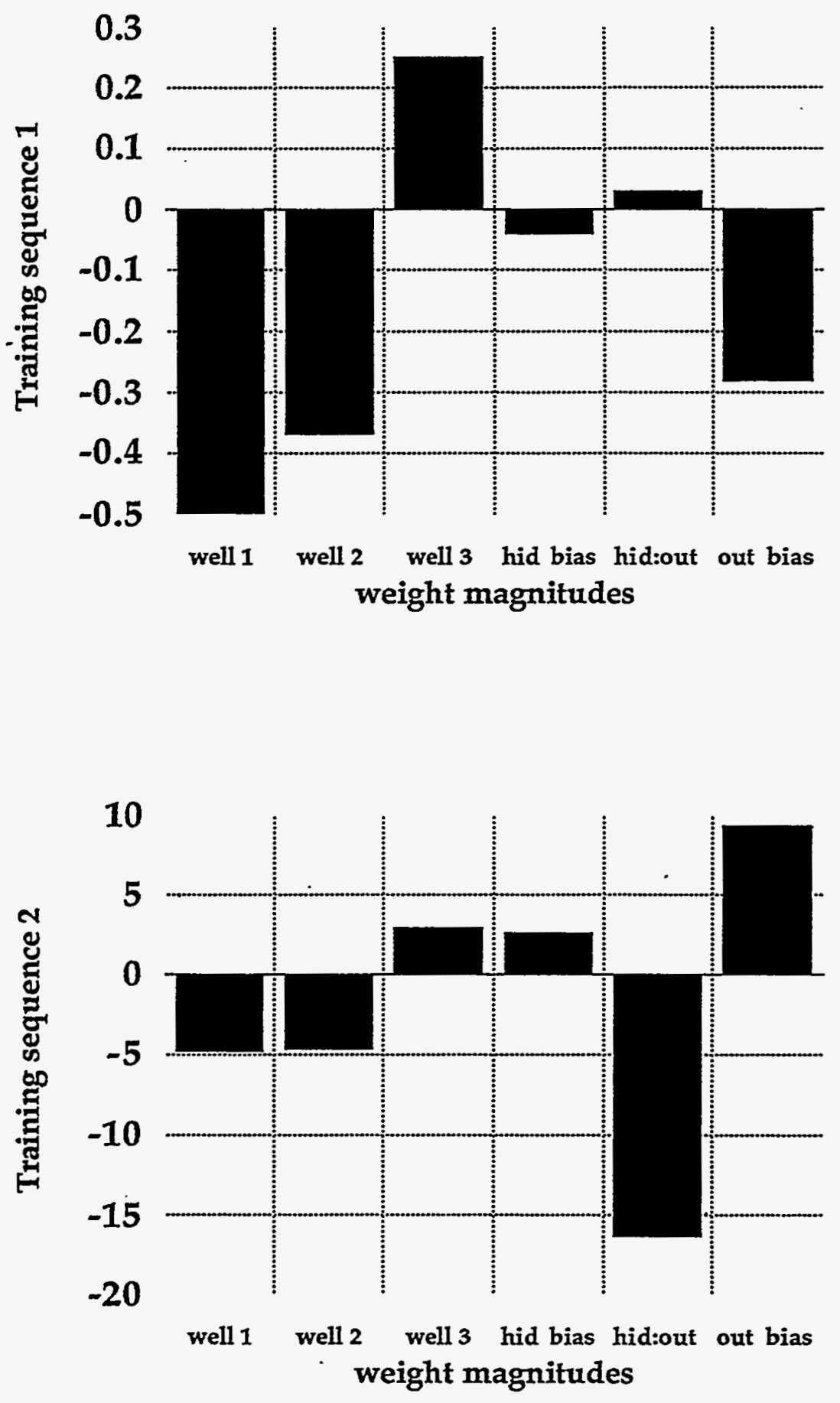

Figure 14. Network weights after training with a) one pattern and b) two patterns. 

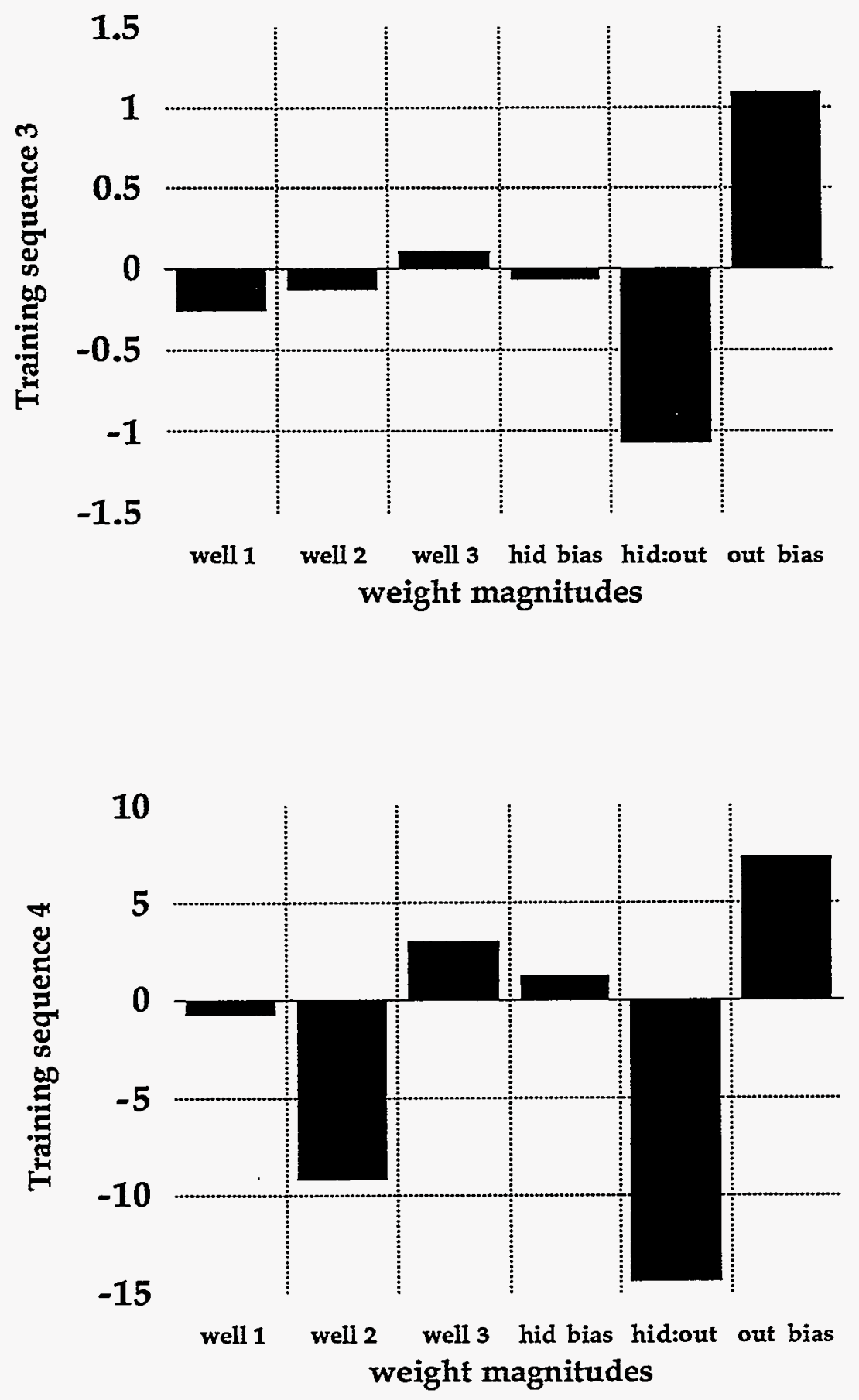

Figure 14. Continued. Network weights after training with c) three patterns and d) four patterns. 
colon is the output value. Understandably its ability to predict the other 7 patterns was not well developed. Its predictions were all .43 (possible range between 0 and 1) for each of the 7 patterns and weights between the three inputs and the hidden layer node were not too dissimilar in magnitude $(-.47,-.31$, and .22$)$ as seen in the first three weights plotted in Figure 14a. Note that the output here is a continuous value and a threshold or cutoff value of .75 is arbitrarily used to distinguish 1 form 0 , with output values less than .75 being considered as 0 and values greater than or equal to .75 being considered as 1 . During the second sequence of training the network was presented with 2 patterns (111: 1 and $001: 0)$. This was enough information for the network to increase the magnitude of the weights $(-5,-5$, and 3$)$. The predictions indicated the network had concluded, if the third input was 1, the outcome would not be successful. This caused the network to predict in error that the pattern (011) would not be successful. Any other combination of well one and two pumping was listed successful, causing it to mistakenly predict the pattern $\left(\begin{array}{ll}0 & 0\end{array}\right)$ was successful. During the third sequence of training the network was presented with 3 patterns (1 1 1:1,001:0, and $110: 1)$. This pattern seemed to move the network back to a more indecisive state as all predictions for the remaining 5 patterns were .64 . During the final sequence of training the network was presented with 4 patterns (the pattern 10 1:0) was added. The information that without the second input the remediation was unsuccessful causes the network to increase the magnitude of the weight between the second input node and the hidden layer to a strong -9 (Figure 14d). The remaining four patterns of pumping $((100,000,011$, and 010$)$ were correctly determined with decisive values of $.1, .02$, .99 , and .99 , respectively. These values are considered decisive as they are quite close to the endpoints of 0 and 1.

This very simple example illustrates a few significant points in weight dissection and maturation. The weights are the mechanism by which the hidden nodes become feature classifiers. The hidden nodes may classify different aspects of the problem depending on the patterns it has been trained with. The different aspects may be such features as visual components, mathematical relationships, or spatial relationships of inputs. The nonlinear nature 
of the sigmoid transform makes it difficult to interpret the relative magnitude of the weights of the network. Note even in this simple example the hidden node bias weight and the weights between the hidden node and output node were quite variable (Figure 14). This is important as we go on to look for appropriate means to quantifiy the relative importance of the weights of a network used to solve the more complicated twenty-well remediation problem.

\subsection{Statistical and Spatial Description of Weights}

For the twenty-well remediation example we will examine the weights associated with the architecture 21-7-1 that resulted from four different series of 195 training patterns out of an original pool of 245 patterns. The learning algorithm used was the conjugate gradient backpropagation without any effort to limit the size of the weights (i.e., no weight elimination). The resulting four series of weights will be referred to as wa, wb, wc, and wd, with the four series referring to series a) $1,2,3,5, b) 2,3,4,5, c) 1,3,4,5$, and d) $1,2,4,5$, respectively.

The first comparison we will look at are the univariate statistics in Table 6. While the means, maximum values, and minimum values are not too dissimilar, one sees differences reflected in such descriptors as the standard deviation, skewness, and kurtosis. These differences reflect that each of the four series of training patterns has arrived at a somewhat different set of weights to describe which wells are most important to a successful remediation. Let us explore this further with spatial plots of the well magnitudes.

Table 6. Univariate statistics for weight sets $w a, w b, w c$, and $w d$.

\begin{tabular}{llllllll}
\hline \hline Wt. Set & No. Wts. & Mean & Variance & Kurtosis & $100 \%$ & $50 \%$ & $0 \%$ \\
wa & 147 & -0.83 & 14.09 & 79.35 & 37.86 & -0.83 & -13.31 \\
wb & 147 & -.0 .71 & 10.12 & 19.48 & 21.96 & -0.57 & -14.25 \\
wc & 147 & -1.21 & 7.02 & 12.52 & 15.45 & -1.64 & -8.87 \\
wd & 147 & -1.55 & 17.57 & 63.88 & 39.40 & -1.57 & -15.93 \\
\hline \hline
\end{tabular}


Figure 15 is a three-dimensional histogram with the $z$ axis reflecting the weight magnitudes (from series wa) feeding into and out of each of the seven hidden nodes of the network. The $x$ and $y$ axis correspond to the areal location of inputs 1 through 20 which represent whether the pumping well at that location is on or off. The three weights which have no areal analogy and are plotted along the $y$ axis close to the origin are 1) the bias weight of the 21st input relating to the parameter representing the percentage of nonpumping wells, 2) the bias weight of the hidden node, and 3) the weight between the hidden node and the output node. With a similar histogram sequence for the weight set, wc, (Figure 16) one sees a fairly different set of weights for the seven hidden nodes. The primary reason for the design of these plots was to view the areal distribution of weights; however, the three weights which have no areal analogy are included so that these plots contain all network weights. The variation in the vertical scale between weight sets makes comparisons more difficult; however, this insures that the relative values within one weight set were able to be seen.

We noted earlier that the best management solutions found by the ANN approach involved consistently pumping certain wells ( $\# 5,10,11,12,13$, and 18), never pumping certain wells $(\# 1,4,6,8,15,17$ and 20$)$, and sometimes pumping the remainder of the wells $(\$ 2,3,7,9$, 14, 16, and 19). We can look at individuall barcharts of weights for each node (Figure 17) and note which of the large magnitude weights are the consistenly, never, or sometimes pumped wells. This can be a qualitative review of whether a particular node has emphasized a certain well. However, the nonlinear sigmoid trasfer function which the summed weights pass through makes a simple large magnitude comparison undependable.

\subsection{Well Sensitivity Analysis}

One might first consider some type of covariance or principal component analysis to reduce the complex associations between the weights, inputs, and outcome of the remediation into a smaller number of components that retained the significant dependencies. However, the weights do not meet the underlying assumptions for such multivariate statistics. Indeed, the 

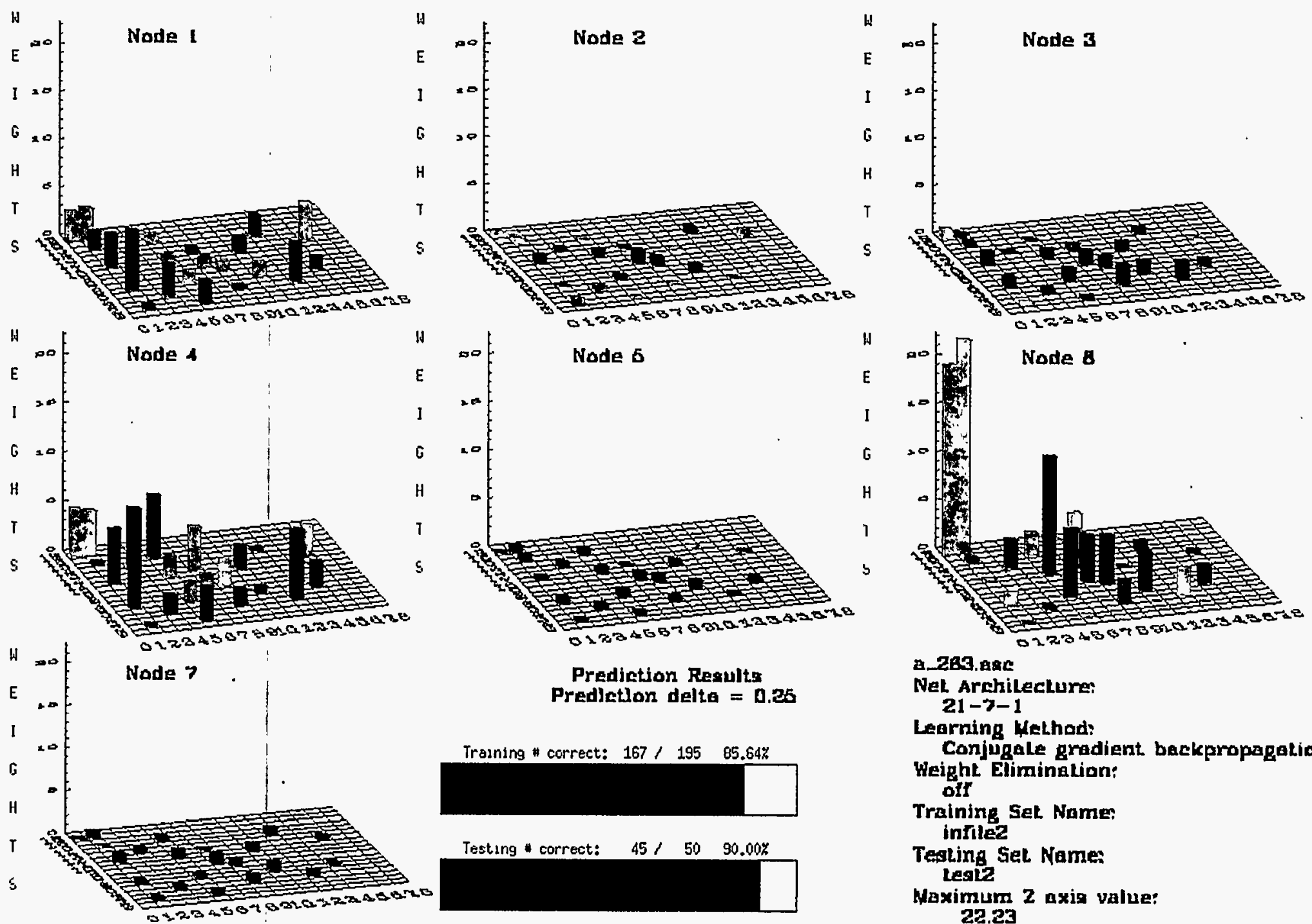

Prediation Regultu Predlation delta $=0.25$

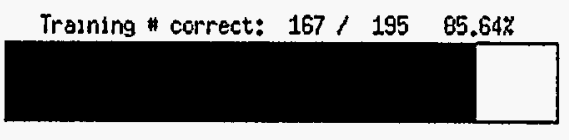

Testıng " correct: $45 / 50 \quad 90.00 \%$

\section{a.Z69.ase}

NeL Arwhilecture 21-7-1

Learning Yethod:

Canjugale gradient backpropagation

Height Eliminakion: olf

Training Set Name infrlez

Teating Set Names

Lesiz

Naximum 2 axis value: 22.23

Figure 15. Performance results and network weights for the architecture 21-7-1 in a three-dimensional historgram for infile 2 with weight set wb. 

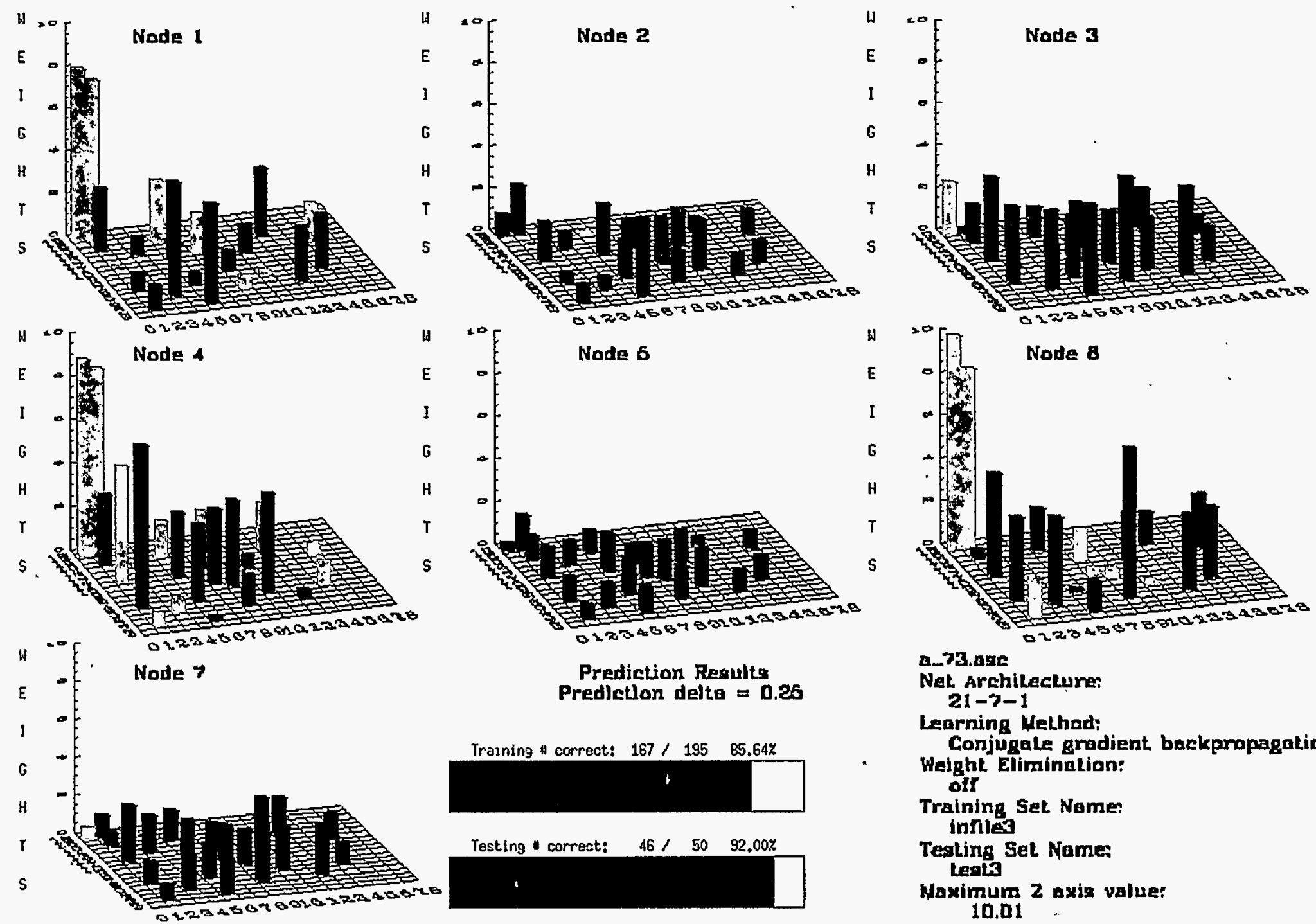

Prediction Resulta

Predlation delto $=0.26$

a.73.ane

Nel Archilectures

$$
\text { 21-7-1 }
$$

Learning Wethad:

Conjugale gradient. backpropagation

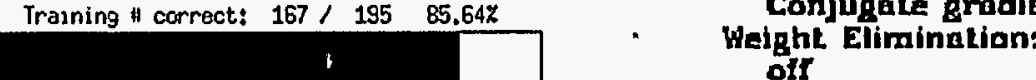

Training Set Name

$$
\text { infilas }
$$

Testing * correct: $\quad 46 / 50 \quad 92.00 z$

Testing Sel Name

Lealo

Maximum 2 nxin value: 10.01 -

Figure 16. Performance results and network weights for the architecture 21-7-1 in a three-dimensional histogram for infile 3 weight set wc. 
Weights Associated with an Individual Hidden Node

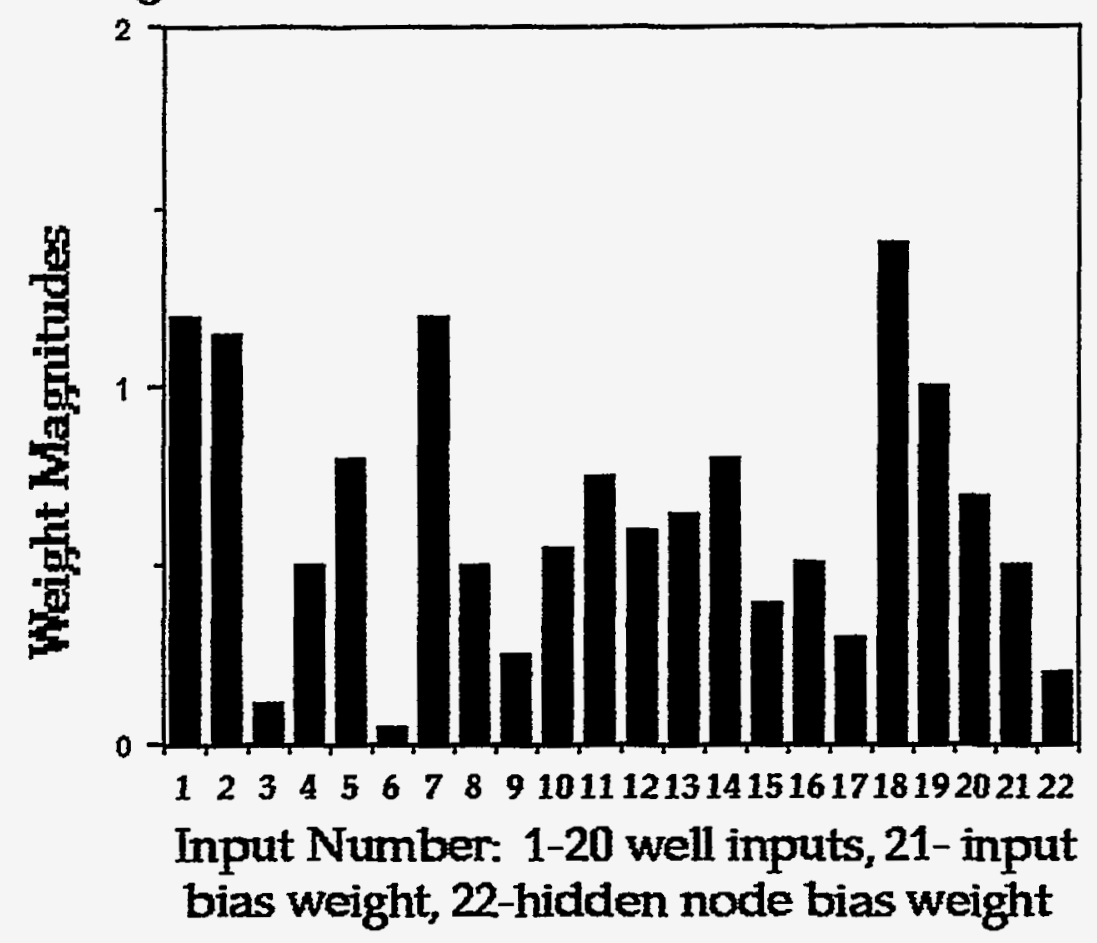

Figure 17. Barchart of network weights for a single hidden node. 
weights themselves can be viewed as already being a distillation of ranked inputs. Thus, a dynamic sensitivity analysis was developed whereby the weights for a particular input were varied by an increasing sequence of perturbations. The percentage of test patterns which had an altered outcome were then recorded. In other words, an altered outcome was recorded if the original weights had produced an output of 1 (or 0 ) and the perturbed weights produced an output of 0 (or 1 ). The effects of perturbations with magnitudes $-10,-5,-4-3,-2,-1,-5,5,1,2,3$, 4,5 , and 10 were examined for all four weight series. This well sensitivity analysis proved to be an appropriate method to capture the relative importance of the various input parameters (i.e., well locations). Let us examine the effects of perturbations of -5 and 5 on all weight series, as shown in Figures 18 and 19. The greatest magnitude of altered outcomes appears in wells 5, 11, 12,13 , and 18 for a -5 perturbation. The 5 perturbation is somewhat of a subdued replica of the magnitude -5 perturbation. Note there is some variation. For example in the 5 perturbation well \#10 is as high in altered outcomes as wells \#12 and \#13 for weight series wc. Also some of the occasionally pumped wells ( $\# 2,7,14$, and 19) scored as high as did the consistently pumped wells (\#5 and \#18) in weight series wc. Figures 19 and 21 illustrate the -10 and 10 perturbation, respectively, for the four weight sets. The -10 perturbation also shows the highest ranking wells in altered outcomes to be $\# 5,11,12,13$, and 18 . Note wells $\# 1$ and 8 which are never pumped wells have more altered outcomes than wells from the sometimes pumped category. Again the positive magnitude (10) perturbation (Figure 21) shows a subdued profile relative to the negative magnitude $(-10)$ perturbation (Figure 20 ). 
Pct of Altered Outcomes by Tweaked Input Vector - 200 Test Cases Defot at Textlog $=-5.0$

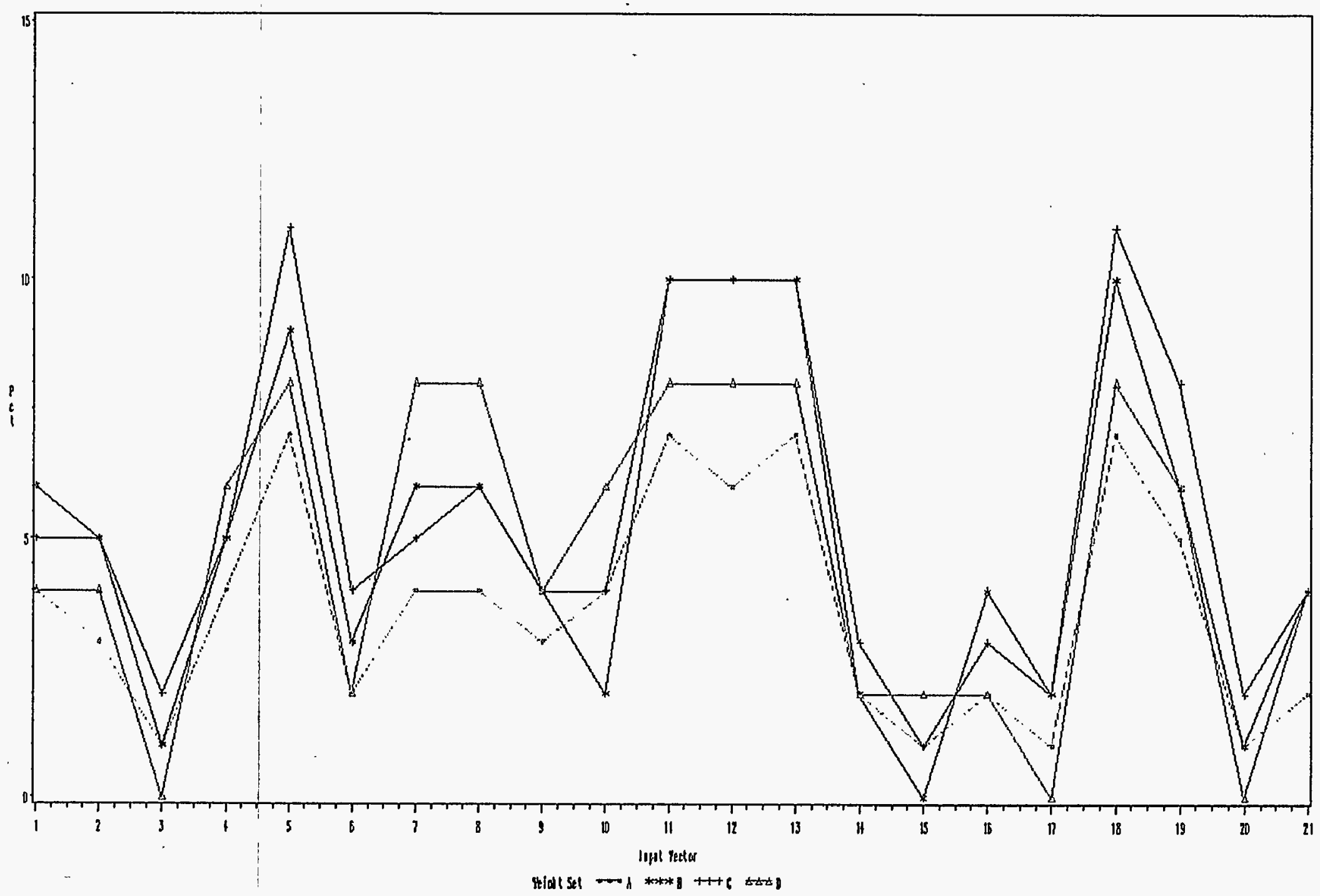

Figure 18. Percentage of altered outcomes by tweaked input vector for -5.0 permutation. 
Pet of Altered Outcomes by Tweaked Input Vector -200 Test Cases

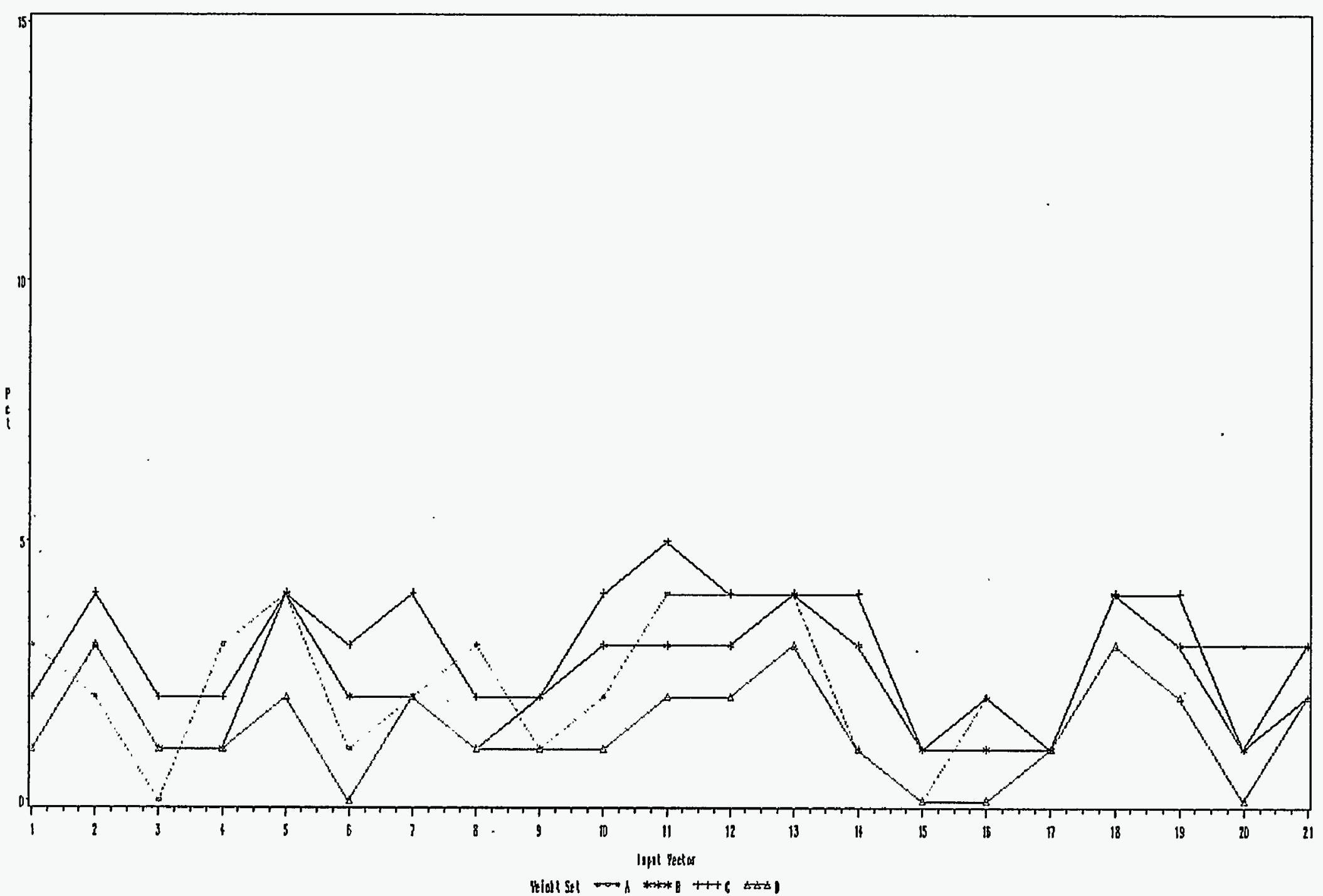

Figure 19. Percentage of altered outcomes by tweaked input vector for 5.0 permutation. 


\section{Pct of Altered Outcomes by Tweaked Input Vector - 200 Test Cases}

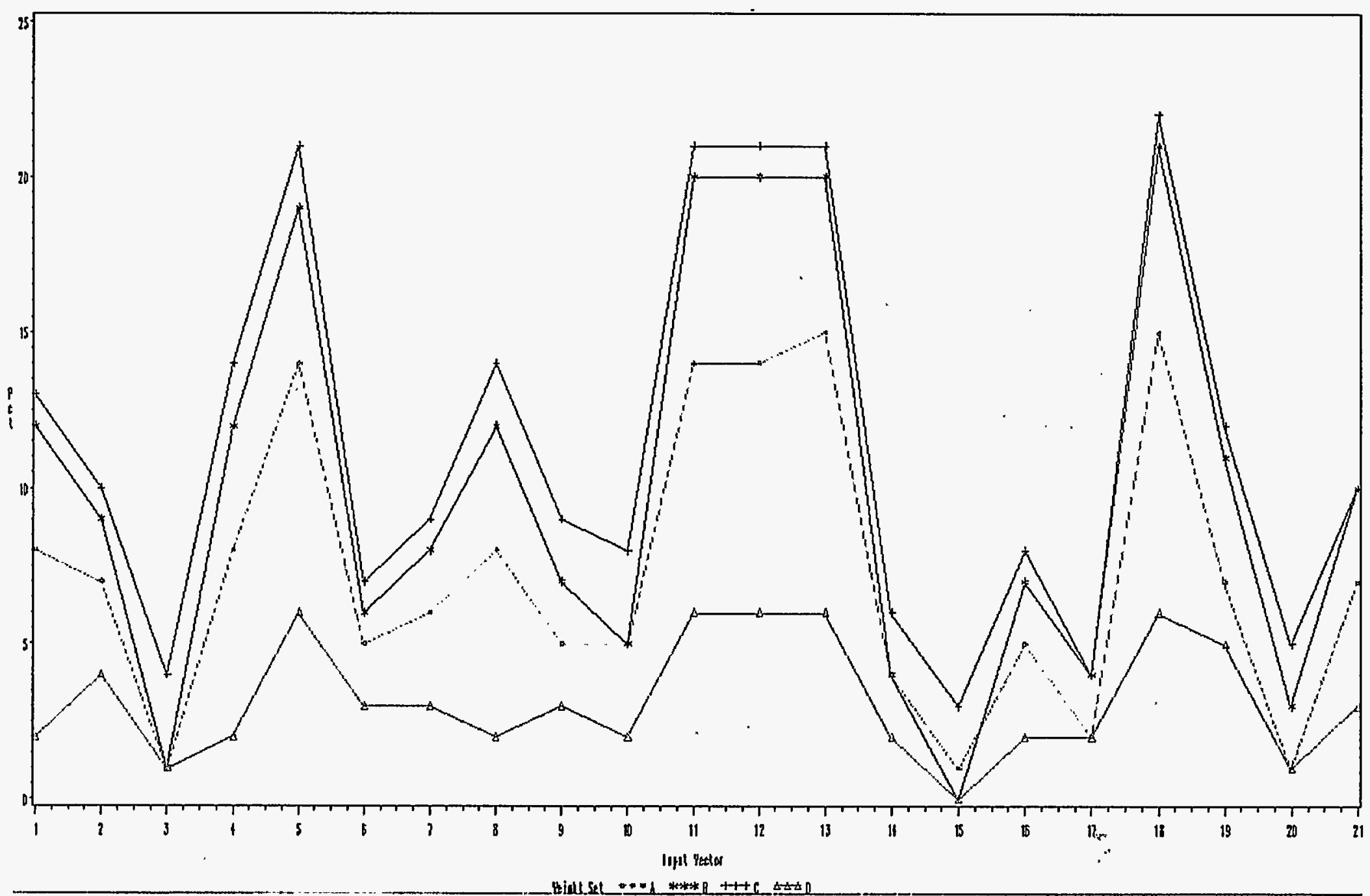

Figure 20. Percentage of altered outcomes by tweaked input vector for -10.0 permutation. 


\section{Pct of Altered Outcomes by Tweaked Input Vector - 200 Test Cases}

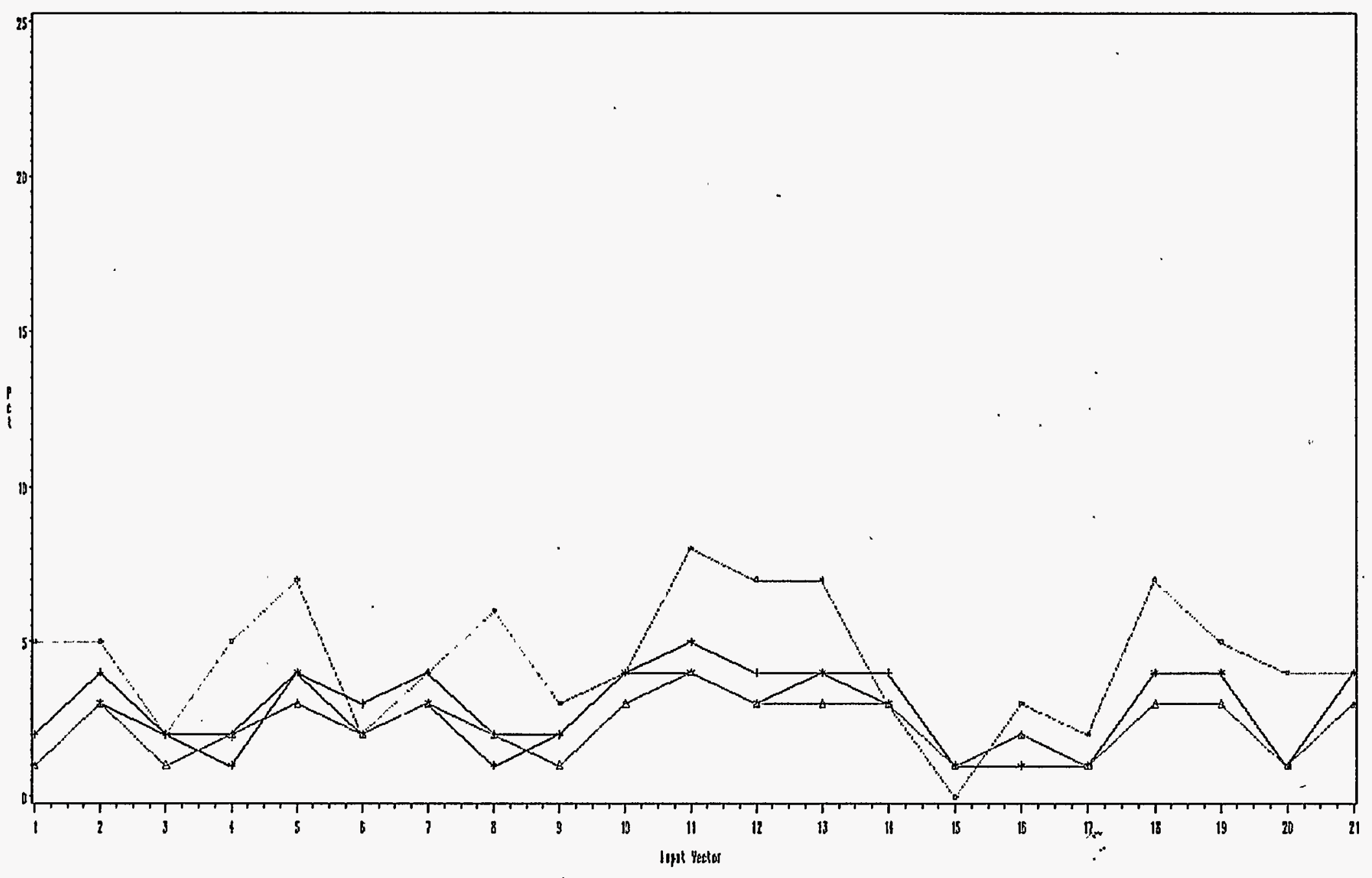

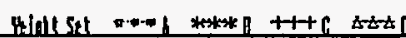

Figure 21. Percentage of altered outcomes by tweaked input vector for 10.0 permutation. 
When rankings of high altered outcomes to low altered outcomes are constructed, the consistently pumped wells do form a cluster in the higher ranks. In Table 7 the wells are in the ranking of high altered outcomes at the top of the table. The perturbation levels of $-1,-5,5$, and 1 are not included because so few altered outcomes occurred that most inputs had a ranking of zero. This well sensitivity weight analysis seems to be a reasonable approach to quantifying which wells figure prominently in the success or failure of remediation. Note that the 21st input parameter scored a medium to low range of altered outcomes (Table 7). This was the parameter representing the percentage of non-pumping wells. We will compare this medium to low ranking with the same parameter in a mass output field-scale application in Section 5.0. 
Table 7a. Rankings of input vector (well) sensitivity for weight set wa.

\begin{tabular}{|c|c|c|c|c|c|c|c|c|c|c|}
\hline \multicolumn{11}{|c|}{ Perturbation Level } \\
\hline Rank & -10 & -5 & 4 & $\underline{-3}$ & -2 & \pm 2 & \pm 3 & +4 & \pm 5 & +10 \\
\hline 1 & 13 & 5 & 5 & 5 & 5 & 11 & 11 & 11 & 5 & 11 \\
\hline 2 & 18 & 11 & 11 & 11 & 11 & 2 & 4 & 4 & 11 & 5 \\
\hline 3 & 5 & 13 & 13 & 12 & 13 & 4 & 5 & 5 & 12 & 12 \\
\hline 4 & 11 & 18 & 18 & 13 & 18 & 5 & 8 & 8 & 13 & 13 \\
\hline 5 & 12 & 12 & 12 & 18 & 2 & 8 & 12 & 12 & 18 & 18 \\
\hline 6 & 1 & 19 & 2 & 2 & 6 & 12 & 13 & 13 & $\mathbf{1}$ & 8 \\
\hline 7 & 4 & 1 & 10 & 10 & 7 & 13 & 18 & 18 & 4 & 1 \\
\hline 8 & 8 & 4 & 19 & 19 & 10 & 18 & 19 & 19 & 8 & 2 \\
\hline 9 & 2 & 7 & $\mathbf{1}$ & 1 & 12 & 19 & 1 & 1 & 19 & 4 \\
\hline 10 & 19 & 8 & 4 & 6 & 14 & 20 & 2 & 2 & 20 & 19 \\
\hline 11 & 21 & 10 & 6 & 7 & 19 & 1 & 7 & 7 & 21 & 7 \\
\hline 12 & 7 & 2 & 7 & 14 & 1 & 6 & 10 & 10 & 2 & 10 \\
\hline 13 & 6 & 9 & 8 & 4 & 4 & 7 & 20 & 20 & 7 & 20 \\
\hline 14 & 9 & 6 & 9 & 8 & 8 & 10 & 6 & 21 & 10 & 21 \\
\hline 15 & 10 & 14 & 14 & 9 & 9 & 14 & 14 & 6 & 16 & 9 \\
\hline 16 & 16 & 16 & 21 & 15 & 15 & 16 & 16 & 14 & 6 & 14 \\
\hline 17 & 14 & 21 & 15 & 16 & 16 & 3 & 17 & 16 & 9 & 16 \\
\hline 18 & 17 & 3 & 16 & 17 & 17 & 9 & 21 & 17 & 14 & 3 \\
\hline 19 & 3 & 15 & 17 & 21 & 21 & 15 & 3 & 3 & 17 & 6 \\
\hline 20 & 15 & 17 & 3 & 3 & 3 & 17 & 9 & 9 & 3 & 17 \\
\hline 21 & 20 & 20 & 20 & 20 & 21 & 15 & 15 & 15 & 15 & 15 \\
\hline
\end{tabular}


Table $7 b$. Rankings of input vector (well) sensitivity for weight set wb.

\begin{tabular}{|c|c|c|c|c|c|c|c|c|c|c|}
\hline & \multicolumn{10}{|c|}{ Perturbation Level } \\
\hline$\underline{\text { Rank }}$ & -10 & -5 & \pm & -3 & -2 & \pm 2 & +3 & \pm 4 & \pm 5 & \pm 10 \\
\hline 1 & 18 & 11 & 5 & 5 & 5 & 2 & 5 & 5 & 5 & 5 \\
\hline 2 & 11 & 12 & 11 & 11 & 11 & 5 & 10 & 13 & 13 & 10 \\
\hline 3 & 12 & 13 & 12 & 12 & 12 & 6 & 11 & 18 & 18 & 11 \\
\hline 4 & 13 & 18 & 13 & 13 & 13 & 10 & 12 & 2 & 2 & 13 \\
\hline 5 & 5 & 5 & 18 & 18 & 18 & 11 & 13 & 10 & 10 & 18 \\
\hline 6 & 1 & 1 & 19 & 2 & 19 & 12 & 18 & 11 & 11 & 19 \\
\hline 7 & 4 & 7 & $\mathbf{1}$ & 19 & 1 & 13 & 19 & 12 & 12 & 2 \\
\hline 8 & 8 & 8 & 2 & 1 & 2 & 14 & 2 & 14 & 14 & 7 \\
\hline 9 & 19 & 19 & 7 & 4 & 4 & 18 & 6 & 19 & 19 & 12 \\
\hline 10 & 21 & 2 & 8 & 6 & 7 & 19 & 7 & 6 & 21 & 14 \\
\hline 11 & 2 & 4 & 4 & 7 & 8 & 4 & 14 & 7 & 6 & 21 \\
\hline 12 & 7 & 9 & 6 & 8 & 10 & 7 & 21 & 9 & 7 & 3 \\
\hline 13 & 9 & 16 & 9 & 9 & 6 & 8 & 1 & 21 & 9 & 6 \\
\hline 14 & 16 & 21 & 16 & 10 & 9 & 9 & 3 & 1 & 1 & 9 \\
\hline 15 & 6 & 6 & 21 & 16 & 14 & 15 & 4 & 3 & 3 & 1 \\
\hline 16 & 10 & 10 & 10 & 14 & 16 & 21 & 8 & 4 & 4 & 4 \\
\hline 17 & 14 & 14 & 14 & 17 & 17 & 1 & 9 & 8 & 8 & 8 \\
\hline 18 & 17 & 17 & 17 & 20 & 20 & 3 & 15 & 15 & 15 & 15 \\
\hline 19 & 20 & 3 & 20 & 21 & 21 & 16 & 16 & 16 & 16 & 16 \\
\hline 20 & 3 & 20 & 3 & 3 & 3 & 17 & 20 & 17 & 17 & 17 \\
\hline 21 & 15 & 15 & 15 & 15 & 15 & 20 & 17 & 20 & 20 & 20 \\
\hline
\end{tabular}


Table 7c. Rankings of input vector (well) sensitivity for weight set wc.

\begin{tabular}{|c|c|c|c|c|c|c|c|c|c|c|}
\hline & \multicolumn{10}{|c|}{ Perturbation Level } \\
\hline Rank & -10 & -5 & -4 & -3 & -2 & \pm 2 & \pm 3 & \pm 4 & \pm 5 & \pm 10 \\
\hline 1 & 18 & 5 & 5 & 5 & 5 & 11 & 11 & 11 & 11 & 11 \\
\hline 2 & 5 & 18 & 18 & 11 & 11 & 12 & 13 & 2 & 2 & 2 \\
\hline 3 & 11 & 11 & 11 & 12 & 12 & 13 & 2 & 5 & 5 & 5 \\
\hline 4 & 12 & 12 & 12 & 13 & 13 & 18 & 5 & 7 & 7 & 7 \\
\hline 5 & 13 & 13 & 13 & 18 & 18 & 2 & 7 & 10 & 10 & 10 \\
\hline 6 & 4 & 19 & 19 & 19 & 19 & 5 & 10 & 12 & 12 & 12 \\
\hline 7 & 8 & 8 & 2 & 7 & 2 & 6 & 12 & 13 & 13 & 13 \\
\hline 8 & 1 & 1 & 8 & 8 & 7 & 7 & .14 & 14 & 14 & 14 \\
\hline 9 & 19 & 2 & 9 & 10 & 10 & 10 & 18 & 18 & 18 & 18 \\
\hline 10 & 2 & 4 & 1 & 1 & 1 & 14 & 19 & 19 & 19 & 19 \\
\hline 11 & 21 & 7 & 4 & 2 & 4 & 19 & 1 & 6 & 6 & 21 \\
\hline 12 & 7 & 6 & 6 & 4 & 6 & 1 & 4 & 1 & 1 & 6 \\
\hline 13 & 9 & 9 & 7 & 6 & 8 & 3 & 6 & 3 & 3 & 1 \\
\hline 14 & 10 & 10 & 10 & 9 & 9 & 4 & 8 & 4 & 4 & 3 \\
\hline 15 & 16 & 21 & 14 & 14 & 14 & 8 & 9 & 8 & 8 & 4 \\
\hline 16 & 6 & 14 & 21 & 3 & 16 & 9 & 16 & 9 & 9 & 8 \\
\hline 17 & 14 & 16 & 3 & 16 & 3 & 16 & 3 & 16 & 16 & 9 \\
\hline 18 & 20 & 3 & 16 & 17 & 15 & 17 & 17 & 21 & 21 & 16 \\
\hline 19 & 3 & 17 & 17 & 20 & 17 & 15 & 20 & 15 & 15 & 15 \\
\hline 20 & 17 & 20 & 20 & 15 & 20 & 20 & 15 & 17 & 17 & 17 \\
\hline 21 & 15 & 15 & 15 & 21 & 21 & 21 & 21 & 20 & 20 & 20 \\
\hline
\end{tabular}


Table 7d. Rankings of input vector (well) sensitivity for weight set wd.

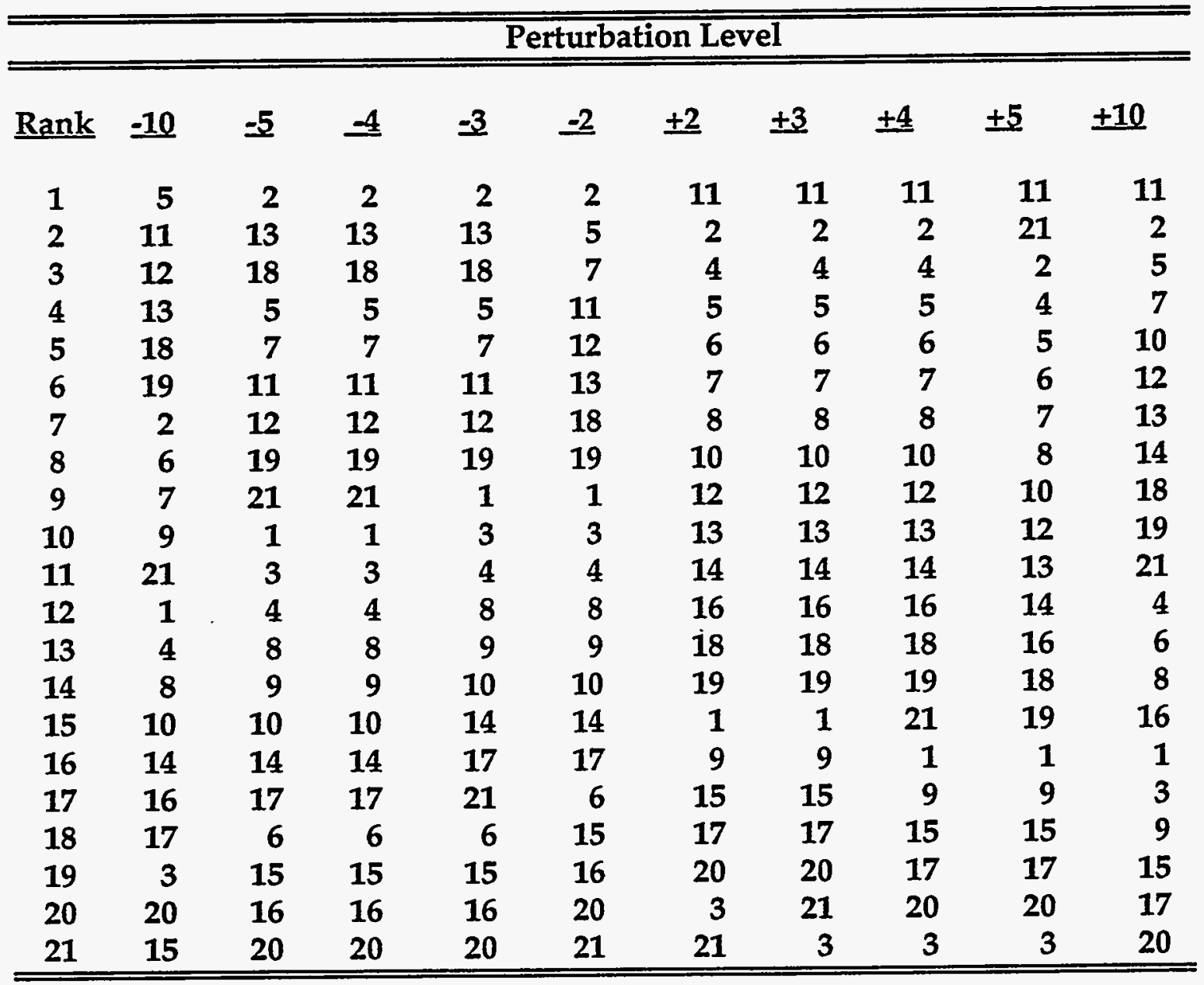




\subsection{Network Complexity}

Histograms in 3-D can be useful in comparing weight magnitudes resulting from penalizing network complexity. The histograms in Figures 22 and 23 show how the weight elimination procedures penalize high magnitude weights in an effort to limit network complexity and thus increase generalization performance. The format is analogous to Figures 15 and 16. Note on the figures that performance of the weights for predicting both training and testing patterns is shown at the lower center of the page. As a brief summary of what the weight elimination procedure actually does, we can compare the weights at the completion of training for a backpropagation learning algorithm that had no penalty for network complexity (Figure 22) with the weights at the completion of training for a backpropagation learning algorithm that did penalize network complexity (Figure 23). The same set of patterns was used for training and testing in both figures. Note that the $z$ axes on these figures are slightly different to better show the weight variations. The weight magnitudes are greater where there was no penalty for complexity. Yet the network trained with a penalty for complexity did in fact do approximately $5 \%$ better at prediction of the test patterns. 

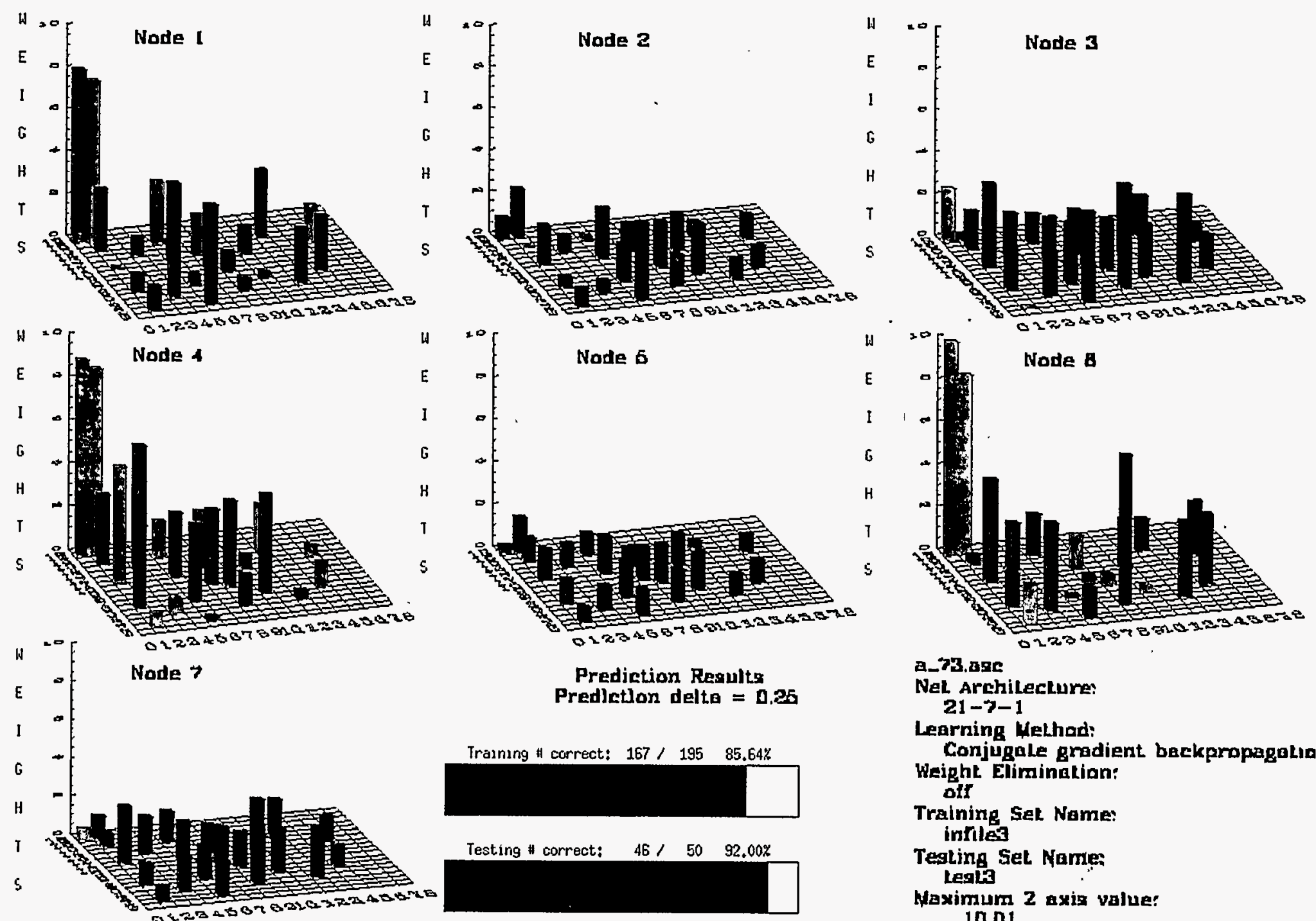

\section{a_73.0도 \\ Nel archilecture: \\ 21-7-1}

Prediction Resulta

Predletion delta $=0.25$

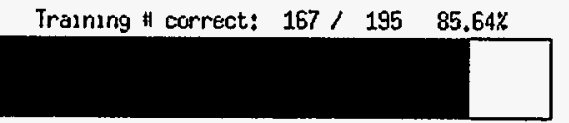

Testing " correct: $\quad 46 / \quad 50 \quad 92,00 \%$

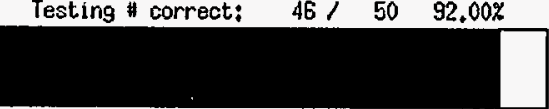

Learning Wethod;

Conjugale gradient beckprapagalian Weight Elimination:

otf

Training Sel Name

infrles

Tegling Set Name:

Maximum 2 axis value 10.01

Figure 22. Performance results and network weights for the architecture 21-7-1 in a three-dimensional histogram for infile 3 with no weight elimination. 


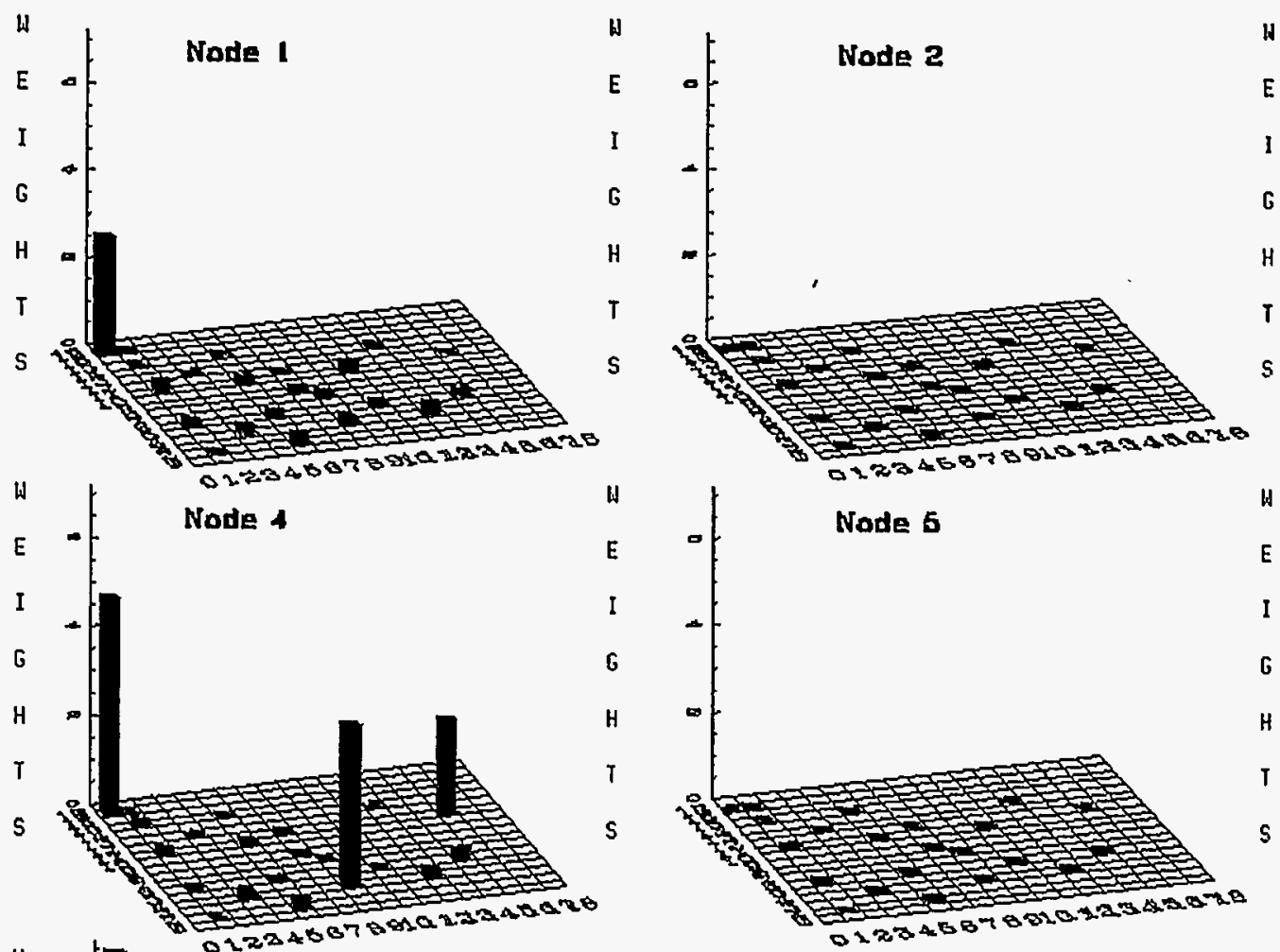

Prediction Reaulto Predjetlan delte $=0.25$

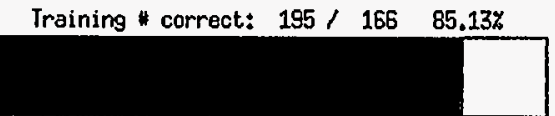

Testing \# correct: $195 / 191 \quad 97,95 \%$

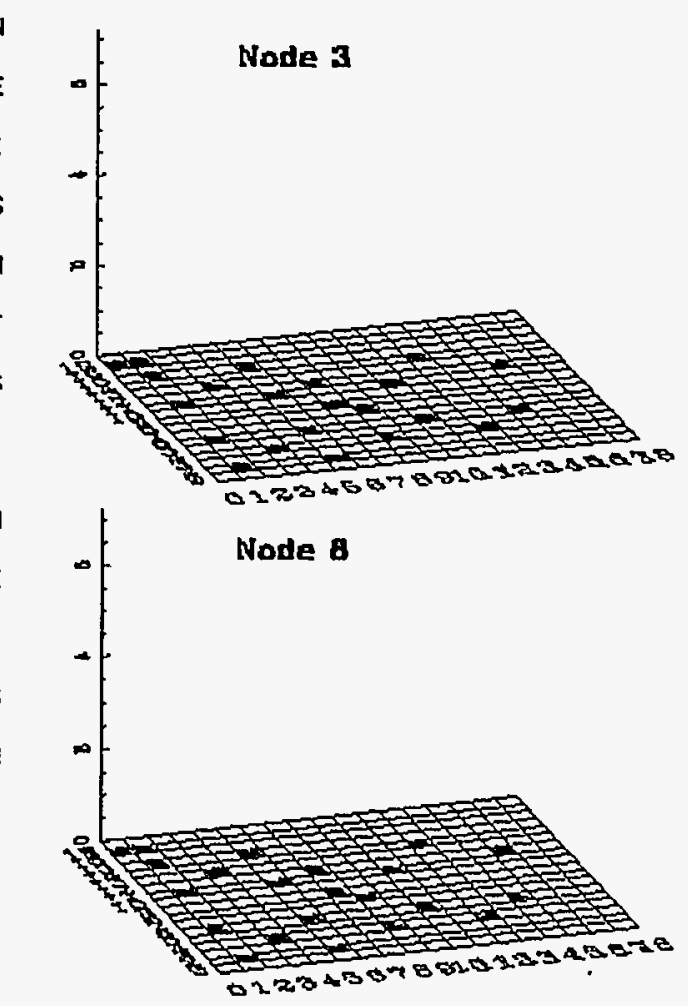

A-ABB.ese

Net Archilecture 21-7-1

Learning Wethad: Conjugale gradient backpropzgatian Weight Elimination:

Training Set Name

innles

Tegting Set Name:

Legti

Naximum 2 axis value:

$$
7.20
$$

Figure 23. Performance results and network weights for the architecture 21-7-1 in a three-dimensional histogram for infile 3 with weight elimination. 


\subsection{Field-Scale Applications}

The computational advantages and performance of the ANN management approach become even more convincing with the increase in the complexity of the flow and transport codes necessary to characterize a field-scale remediation problem. Presented here are examples from remediation activities at Lawrence Livermore National Laboratory (LLNL).

LLNL is a well-charcterized site which was added to the National Priorities (Superfund) List in 1987. Groundwater and vadose zone contaminants of volatile organic compounds (VOCs), tritium, and gasoline are of primary concern. The plume is conceptualized in an areal 2D approach with vertical integration of hydraulic parameters. The LLNL area is underlain by several hundred feet of complexly interbedded, unconsolidated alluvial sediments of Late Tertiary and Quaternary Age. Pumping-test results in the vicinity of the PCE plume suggest the zones being pumped were confined aquifers with leaky behavior. Composite isoconcentration contours of vertically integrated total volatile organic compounds show two primary areas of contamination, one in the central part of the site and one at the southwest comer of the site (Figure 24a). A detailed description of the field site is not given here. Readers are referred to the LLNL Remedial Investigations and Feasibility Studies (Thorpe et al., 1989), as well as Dresen et al.(1987), Rogers (1990, 1991, 1992) Tompson et al., (1991).

\subsection{Predicting Mass Output}

A more complex field-scale remediation goal of maximizing contaminant mass output pumped was addressed in this application. Twenty-eight remediation well locations were selected over the LLNL site for pump-and-treat remediation simulations (Figure 24b). The ANN 


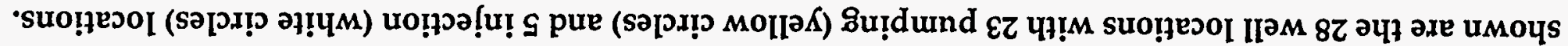

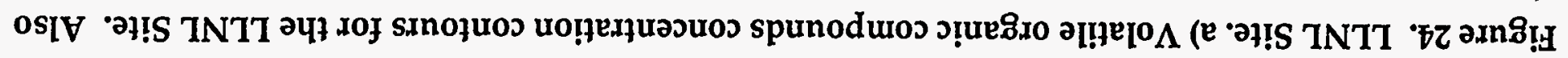

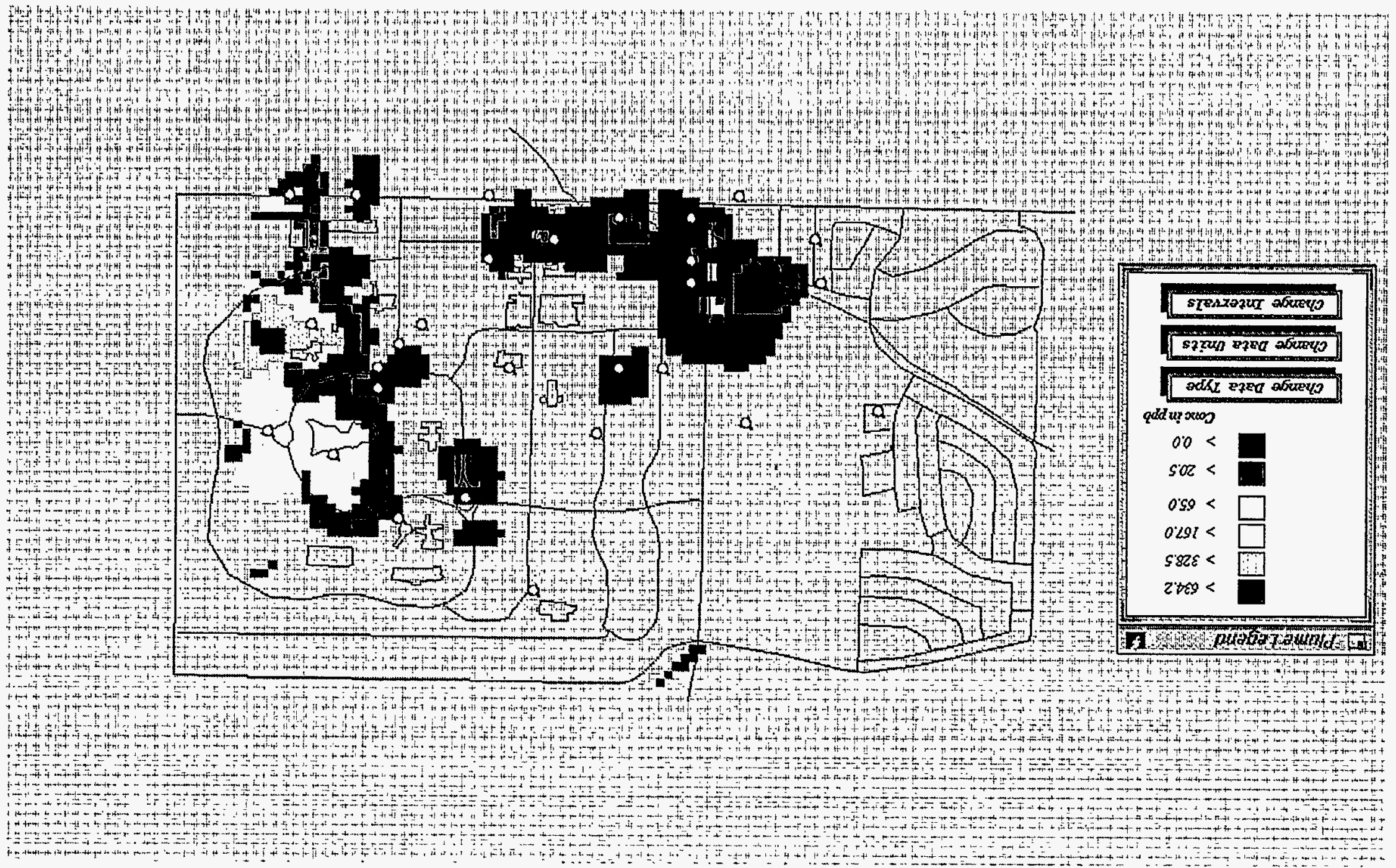




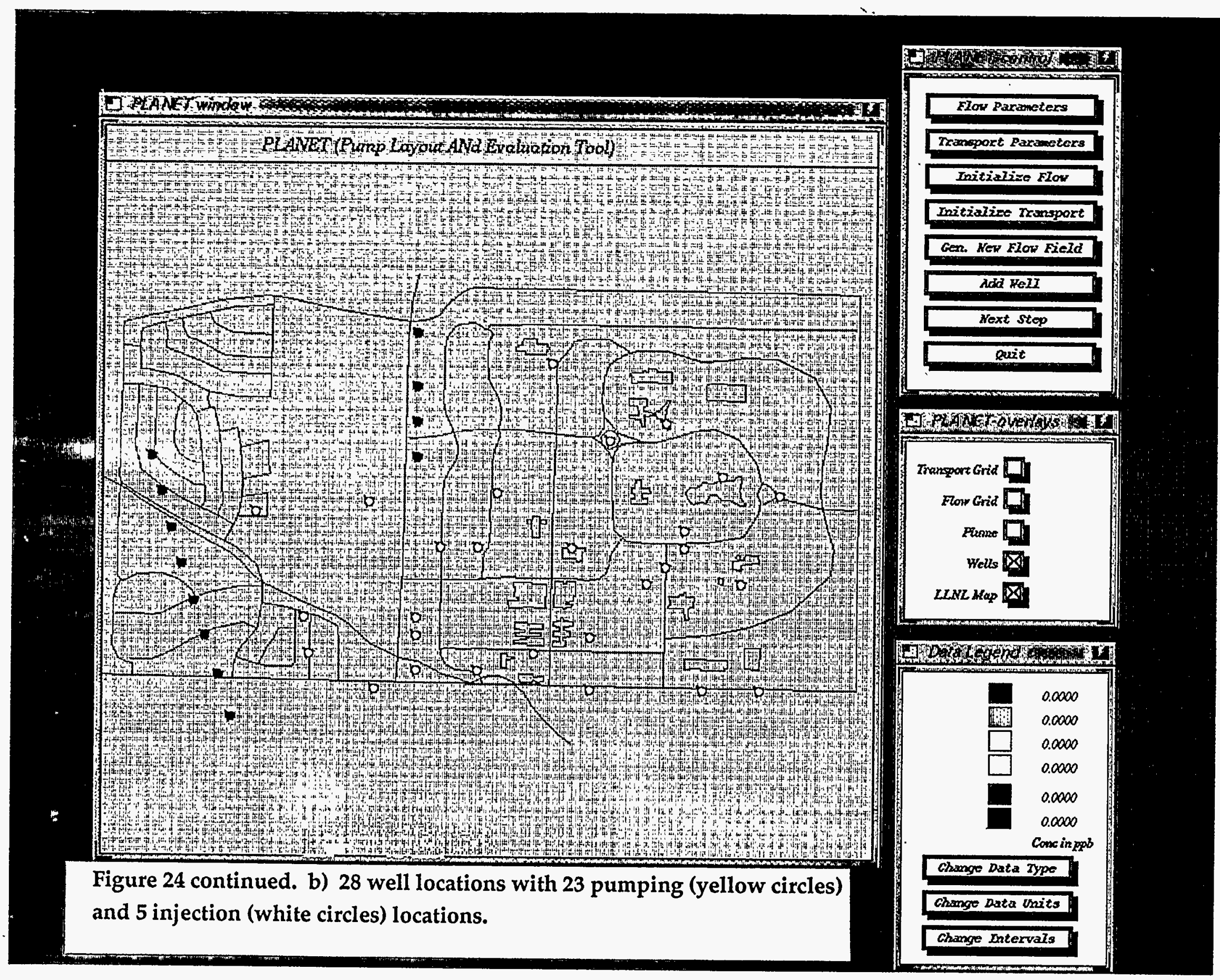


was trained on patterns or realizations of pumping with each pattern having 28 components representing the individual wells, each either 1 or 0 (i.e., discrete levels, 0 being off and 1 being on at the maximum well capacity). Of the 28 wells, 23 were extraction wells and 5 were injection wells, represented by yellow and white circles respectively in Figure 24. The maximum well capacities were determined from lithology and long-term pumping tests in existing wells which were close to the well locations. In several well locations there were already exsisting wells which had been tested. The actual contaminant mass which would be removed from a particular pattern was determined with the aid of a 2-D flow and transport simulator PLANET (Canales et al., 1992).

PLANET (Pump Layout And Evaluation Tool) was developed at LLNL to facilitate relatively rapid interactive diplay of the migration of saturated zone contaminants. At this point in its development PLANET consists of 1) a calibrated, pseudo three-dimensional, finite difference flow code, MODFLOW, 2) a reactive, two-dimensional particle tracking model (SLIM), and 3) a map-oriented graphical interface. The flow processes (MODFLOW) were modeled in two dimensions within a 25 square-mile area surrounding LLNL. The regional grid is composed of 29,673 rectangular cells. Four hydraulic conductivity values were used to represent four main hydrogeologic units with a typical value being $3 \mathrm{~m} / \mathrm{day}$. The boundary conditions included specified head and flux values, several influent and effluent streams, several municipal and irrigation wells, and constant annual recharge from rainfall. A steady flow model was originally calibrated to the pieziometric surface, which has been fairly constant in topology, although during the 1976-78 drought water levels were approximately 3 to $5 \mathrm{~m}$ lower across the basin than pre-drought and present levels.

The two-dimensional transport simulation (SLIM) involved an initial source of approximately $800 \mathrm{~kg}$ of mass distributed between 40,000 particles throughout the domain. Effective dispersivities on the order of 3 to $30 \mathrm{~m}$ were used. The reader is referred to Canales et al. (1992) and Tompson et al. (1991) for more detail. 
The flow and transport simulations were run initially for 225 pumping realizations over 40 years. The 40 year time interval is referred to as a management period, or length of time over which the contaminant transport will be managed. The length of 40 years was chosen because this is the length of time during which unremediated contamination would pass through residential areas judged to need protection. These 225 simulations provided the "answers", or best predictions available for actual results of implementation of pumping realizations, with which to train and test ANNs. The set of 225 patterns was derived from 150 insight patterns and 75 randomly generated patterns of the 28 wells being on or off. The 150 insight patterns were constructed with initial patterns of wells close to areas of high contaminant concentration being on; later different sequences of wells further from the "hot spots" were added. These patterns had a range between 0 and 5 injection wells being active. Two different splits of these 225 were used for training an ANN with 175 patterns and testing on the other 50 patterns. A minimum of 50 testing patterns was considered desirable to have a large enough test distribution. The 175 patterns left for testing were slightly more than the number of weights necessary to solve the problem with 5 hidden nodes. This problem with 5 hidden nodes would have the architecture of 29-5-1 and require 156 network weights. The number of training patterns, 175 , was considered less than optimal for increasing the number of hidden nodes to 7 and then 9 . This problem with 7 and 9 hidden nodes requires 218 and 280 network weights, respectively. As it turned out, the ability of the ANNs to predict the 50 patterns they had not seen was quite good in both sets (Figure 25: 175a and Figure 26: 175b) for architectures of 5,7, and 9 hidden nodes. The complete network architectures would be 29-5-1, 29-7-1, and 29-9-1, respectively. Mass values are shown in kilograms normalized to 190 kilograms and scaled between .1 and .9. The mass values were normalized to 190 kilograms because that was the maximum mass removed in the first 225 patterns. The scaling to less than 1 allowed for the ANN to predict that some patterns of pumping it had not been trained on could pump out more mass than the patterns it had been trained on. In other words, the scaling of the training patterns to between .1 and .9 allowed for a 


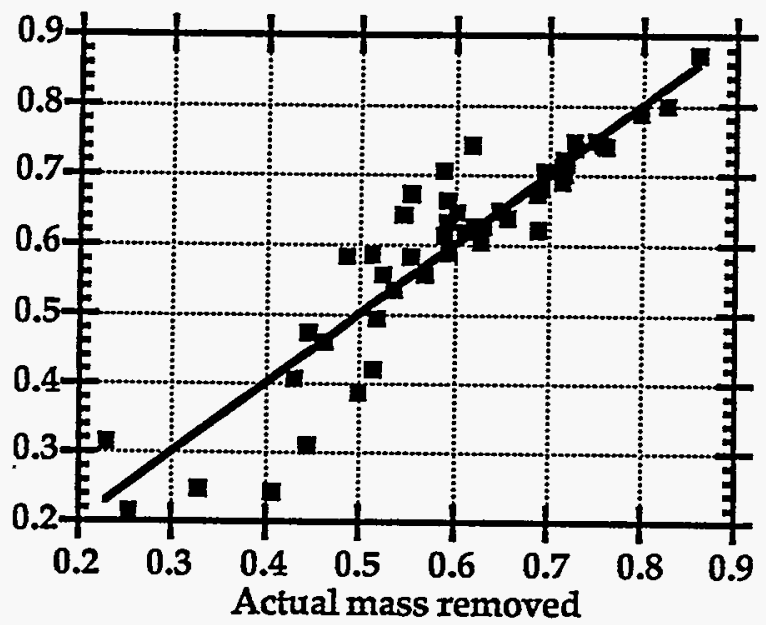

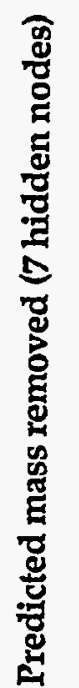

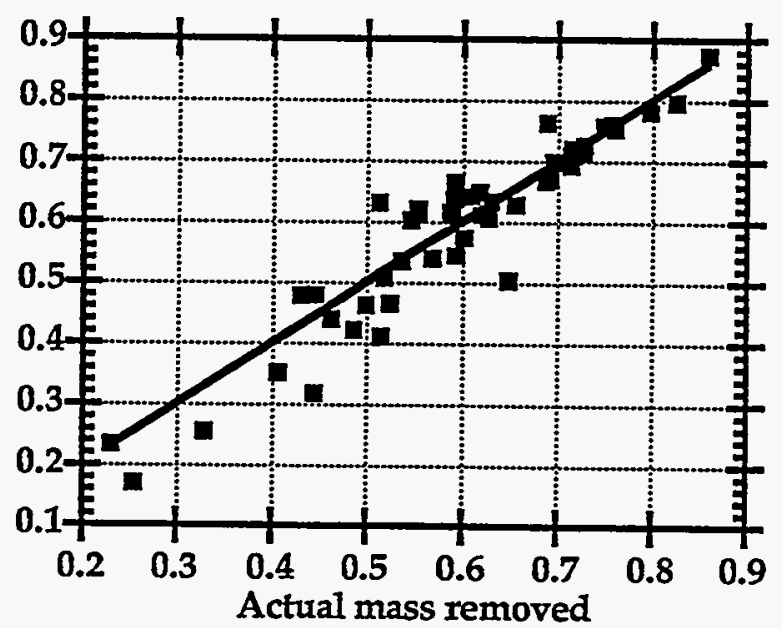

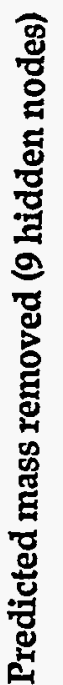

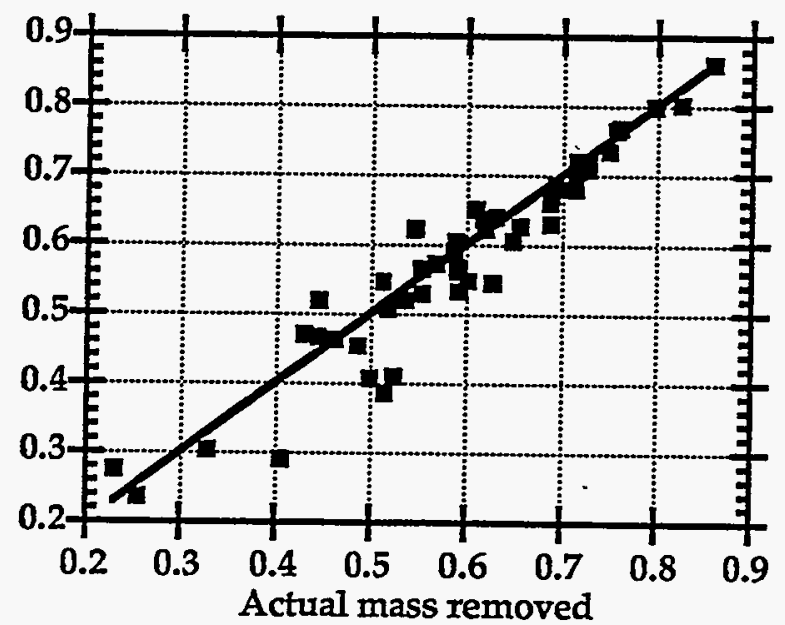

Figure 25. Predicted versus actual mass removed from $\mathbf{5 0}$ test pumping patterns for network trained with set 175a and architecture of a) 29-5-1, b) 29-71, and c) 29-9-1. Mass in $\mathrm{Kg}$ is normalized. 


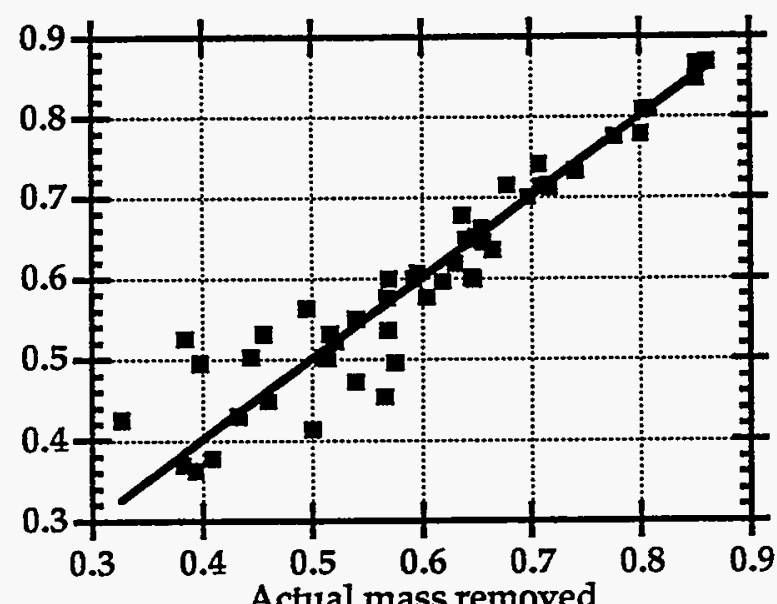



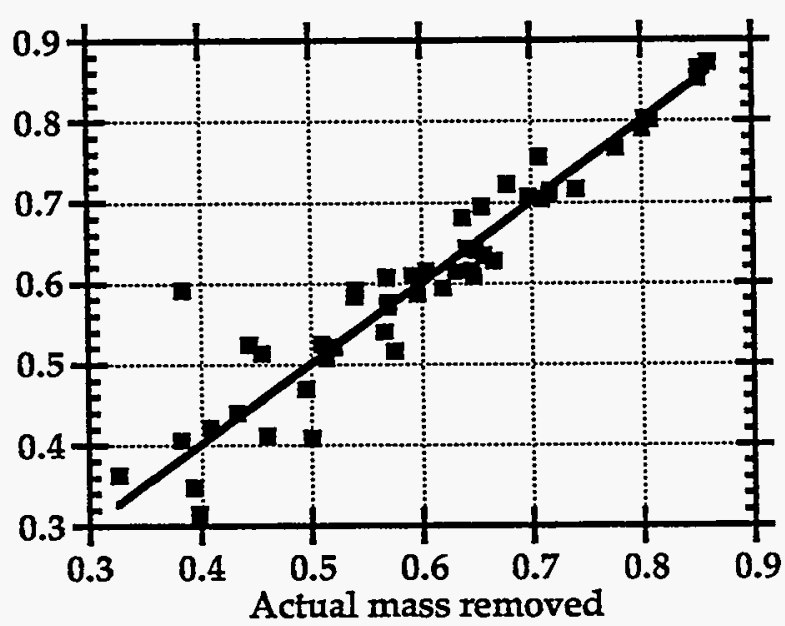

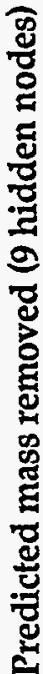

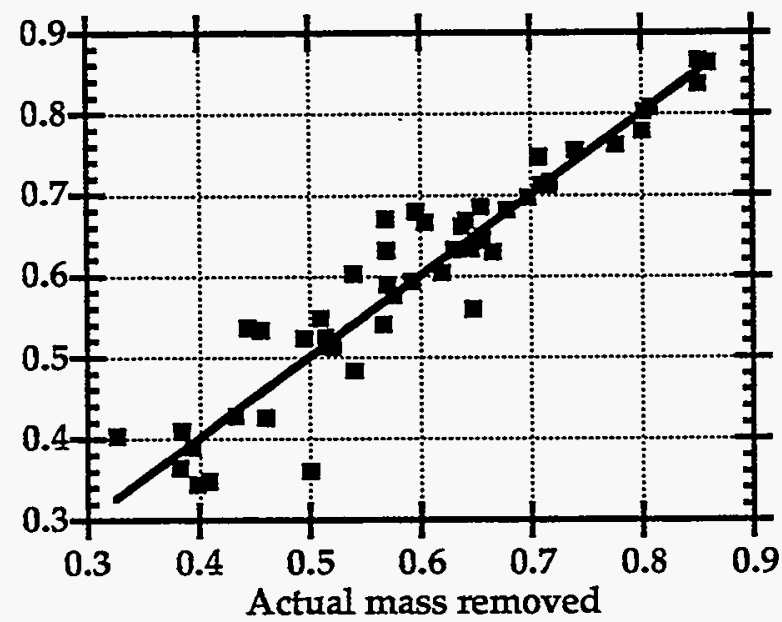

Figure 26. Predicted versus actual mass removed from 50 test pumping patterns for network trained with set 175b and architecture of a) 29-5-1, b) 29-71 , and c) 29-9-1. Mass in $\mathrm{Kg}$ is normalized. 
buffer zone on either extreme for the ANN to generalize that a pattern was above or below the range of mass pumped in the training set.

Plots of the residuals, the difference between "actual" simulated mass output and the mass output "predicted" by the ANN, of these two tests sets show that the performance was better on the first 25 of the 50 patterns for each architecture (Figures 27 and 28). The first 25 patterns were "insight" developed patterns while the second 25 were randomly generated. The sum and mean of the absolute value of the residuals for the various architectures suggest a general improvement in performance with increasing numbers of hidden nodes (Figures 29 and 30).

The trained network of set175a was used to predict the mass output for 2000 different patterns. Like the twenty-well example problem described in Section 3.0, the first 1000 pattems were generated by variations on the training pumping patterns, particularly those judged to be fairly successful, i.e., in the case of an objective to maximize mass output, a training pattern which had a high mass output was judged to be successful. The second 1000 patterns were generated with the GA using a fitness objective that minimized the number of wells operating. Note that this fitness measure can be determined a priori, and thus does not need to call the ANN for an evaluation of the fitness measure. Using the GA in this manner is not a direct optimization of mass output, but rather a generation of additional patterns which seek to find maximum mass output with the fewest number of remediation wells.

In a fraction of the time it would have taken to run a flow and transport simulation for these remediation well scenarios, the ANN was able to predict the mass output for these 2000 patterns. Predictions were made by the ANN for the entire set of 2000 patterns in less than 5 seconds. Note that an actual simulation for one pattern would have taken 20 minutes, and 2000 simulations would have taken 666 hours or approximately 28 days. The patterns for the highest 200 mass outputs predicted by the ANN were run back through PLANET. An injection well has the potential to dilute the contamination, thus causing lower actual mass removed from downgradient extraction wells, if the pumping rates are the same in the original and diluted 
Mass Output Residuals from 175a
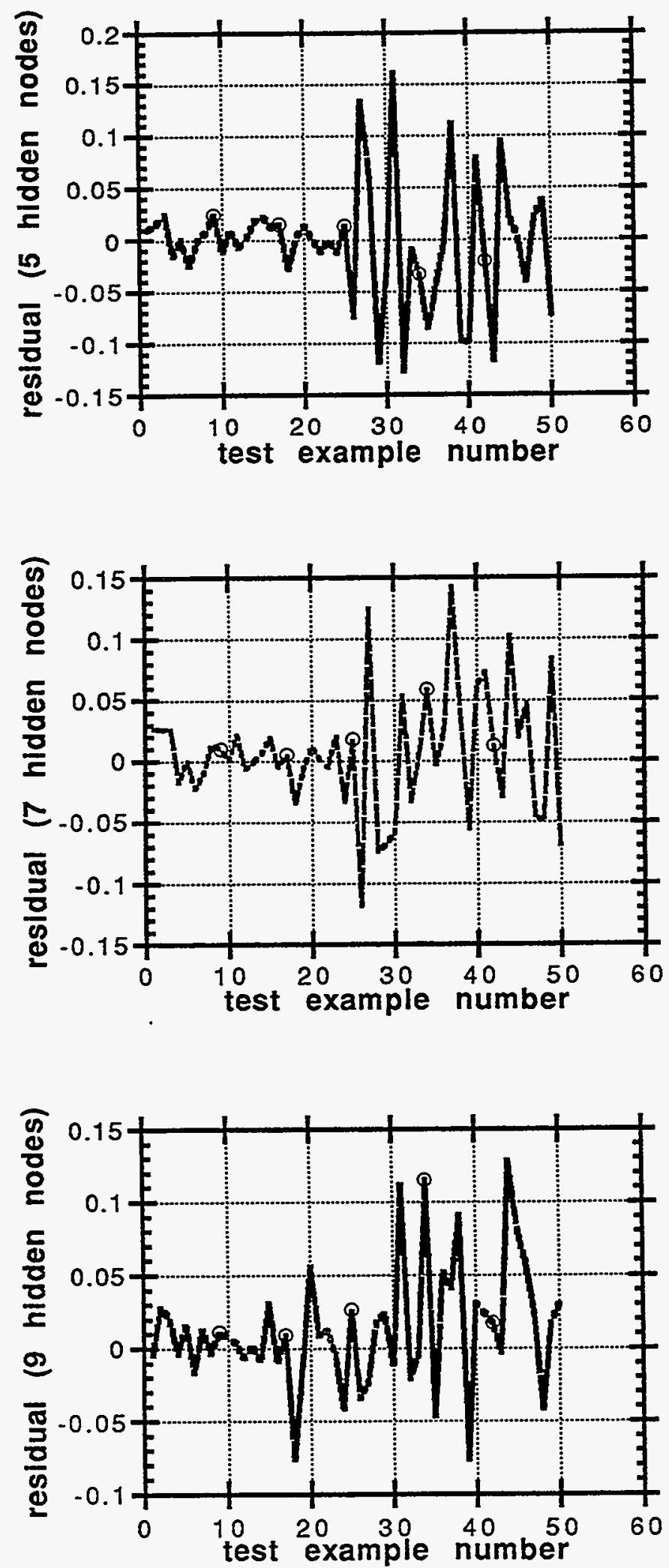

Figure 27. Residuals between predicted and actual mass removed from 50 test pumping patterns for network trained with set $175 a$ and architecture of a) 29-51, b) 29-7-1, and c) 29-9-1. 
Mass Output Residuals from $175 b$
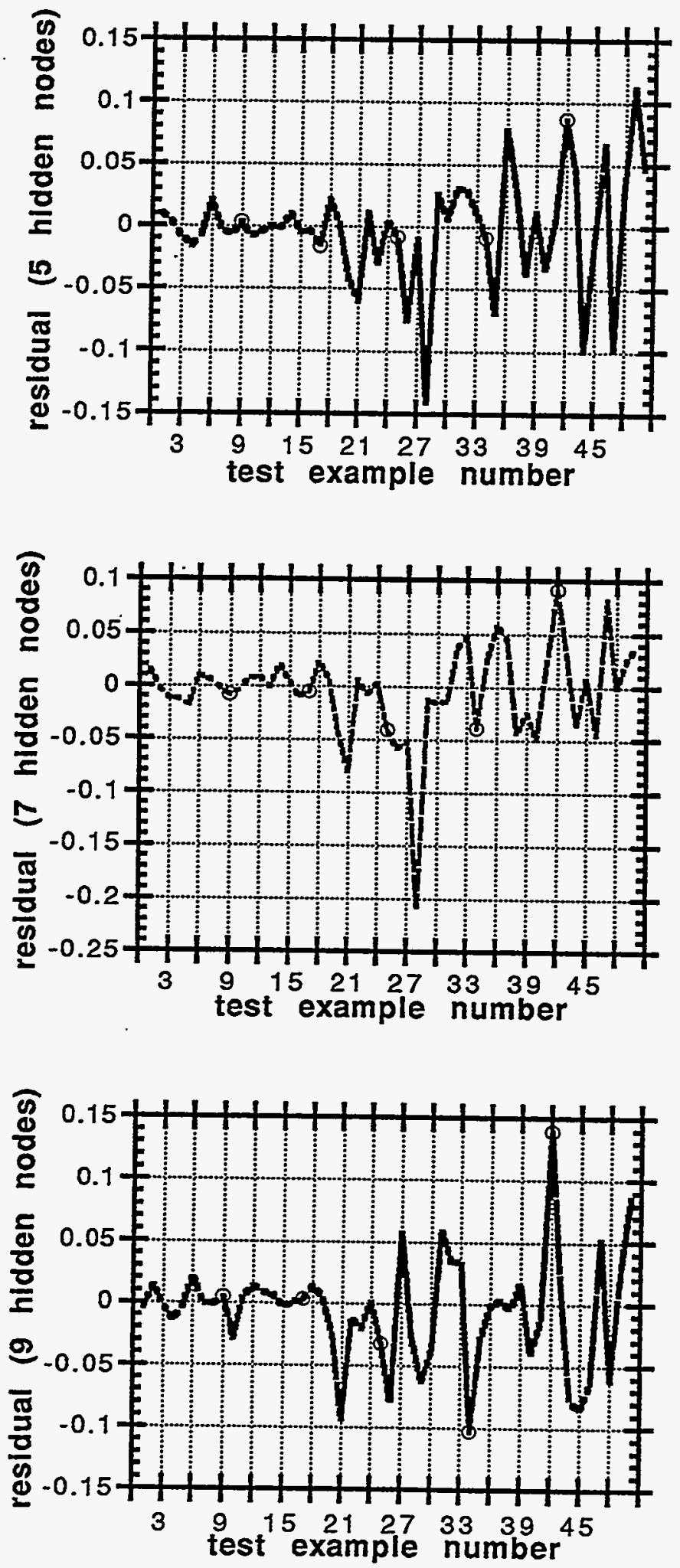

Figure 28. Residuals between predicted and actual mass removed from $\mathbf{5 0}$ test pumping patterns for network trained with set $175 \mathrm{~b}$ and architecture of a) 29-51, b) 29-7-1, and c) 29-9-1. 


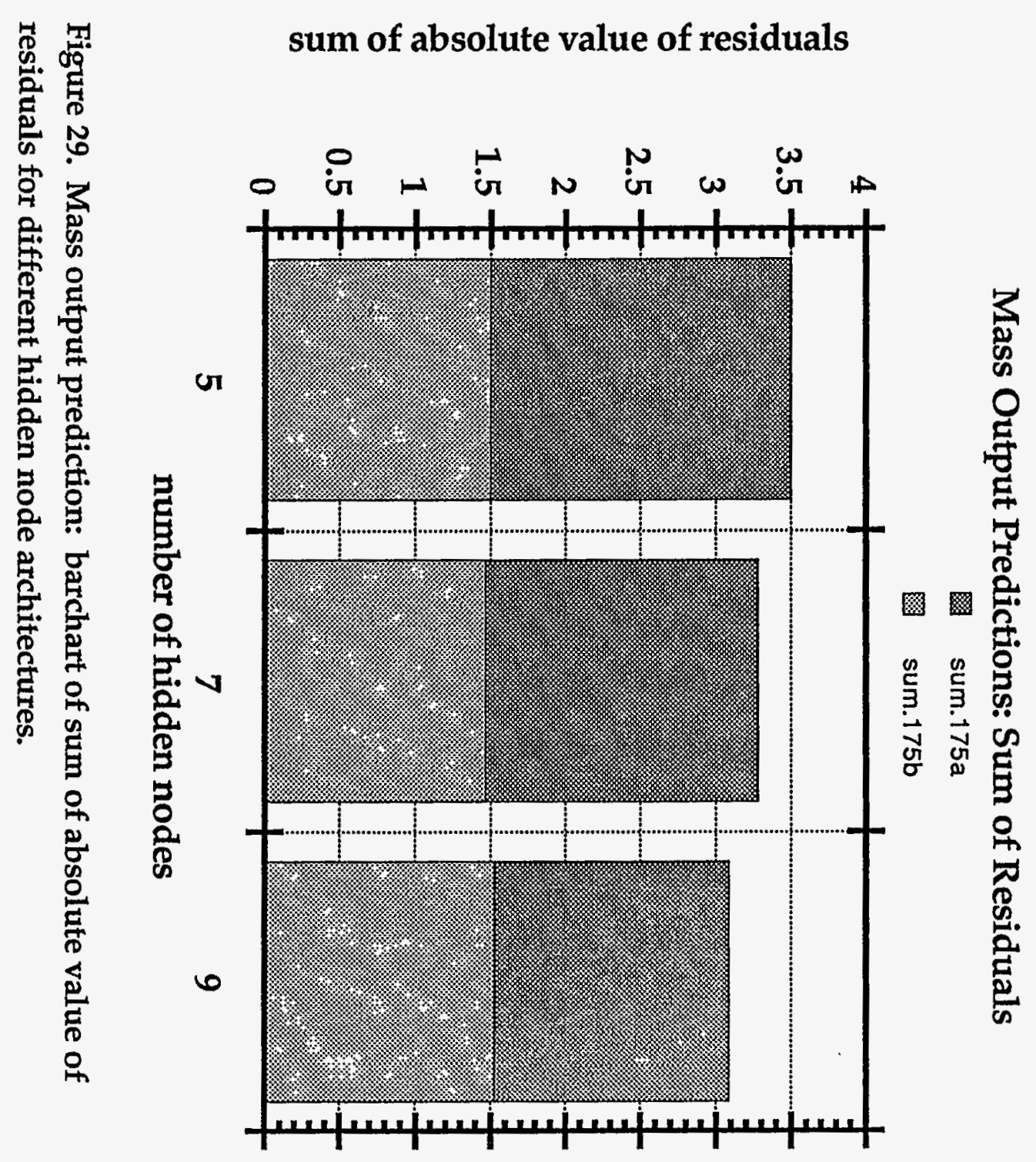




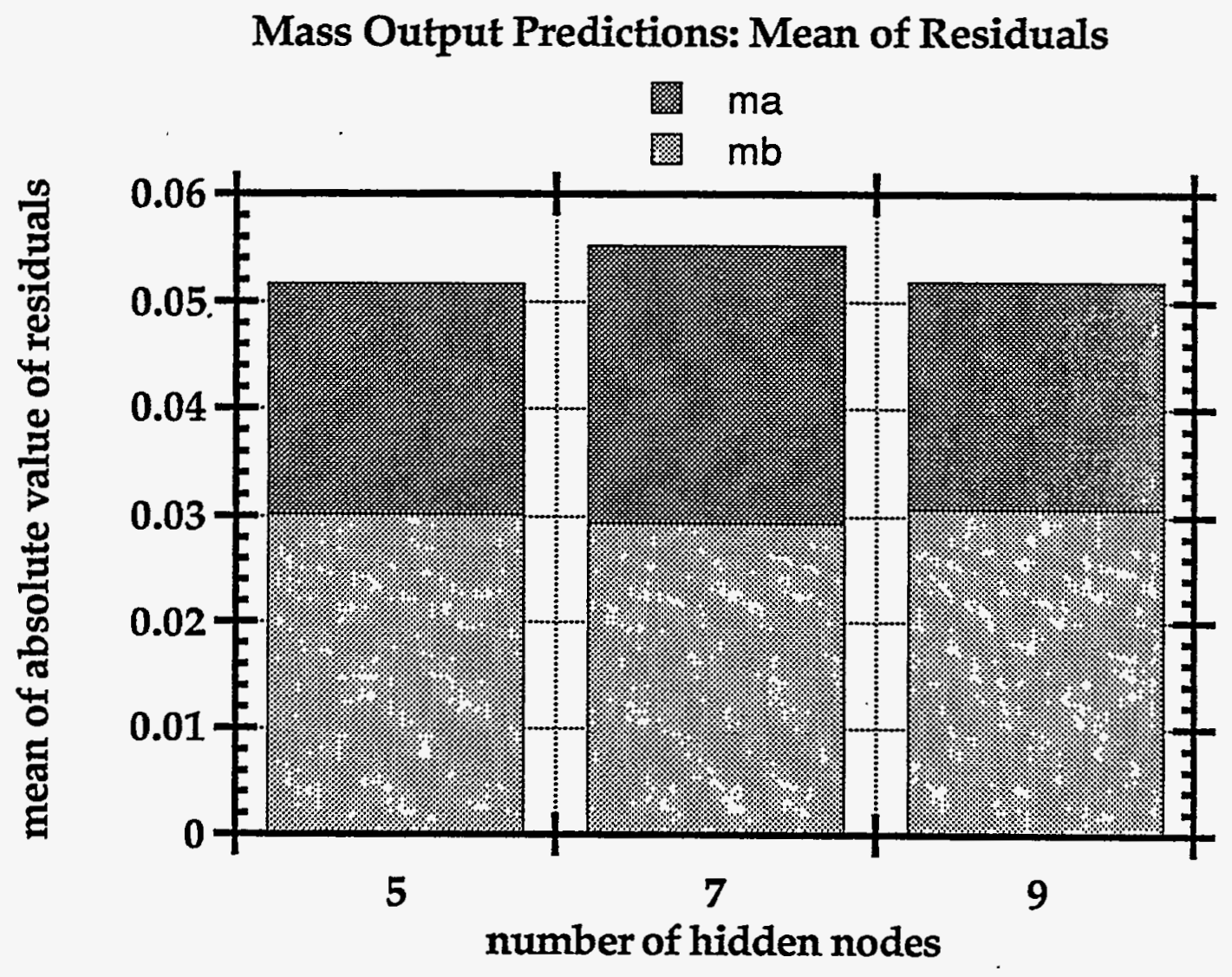

Figure 30. Mass output prediction: barchart of mean of absolute value of residuals for different hidden node architectures. 
scenarios. The ANN appeared to have learned that the injection wells did not help increase mass output, and none of the 200 patterns predicted by the ANN to produce the greatest mass output invovled injection wells turned on. Approximately 25 patterns of the 200 were found to have mass outputs greater than if all 23 pumping wells were on. Note that the pattern of 23 pumping wells and no injection wells was one of the training runs.

A well sensitivity analysis of perturbated network weights, like that described in Section 4.3, was done for this mass output problem. Rankings of input vector (well) sensitivity (Table 8) suggested the wells located in the centers of mass were important in the ANN decisions on mass removed. Some of the wells which figured highly in the rankings were well numbers $1,2,3,4,8$, 19 , and 20 (Figure 31). The first four are in the central area of contamination, the last two are in the southwestern plume, and well number 8 is right in between both of these major plumes being downgradient from the central area plume.

In summary, this is an extremely complex nonlinear problem of wells competing for contaminant removal over a simulation period of 40 years, yet the problem was tractable for the ANNs. Note that this objective of maximizing mass output was a continuous one. Through the normalization and scaling process, the trained ANN was able to predict that some of the 2000 patterns presented to it were as high if not higher than any mass output value the ANN had been exposed to in training. 
Table 8a. Rankings of input vector (well) sensitivity for weight set 175 wa7.

\begin{tabular}{|c|c|c|c|c|c|c|c|c|c|c|c|c|c|c|}
\hline \multicolumn{15}{|c|}{ Perturbation Level } \\
\hline \multicolumn{2}{|c|}{ Rank $=10$} & -5 & -4 & $\underline{3}$ & -2 & -1 & $\underline{-.5}$ &, \pm 5 & \pm 1 & \pm 2 & +3 & +4 & \pm 5 & +10 \\
\hline 1 & 29 & 29 & 29 & 1 & 1 & 1 & 2 & 2 & 2 & 2 & 2 & 2 & 2 & 29 \\
\hline 2 & 1 & $I$ & 1 & 2 & $\overline{2}$ & 2 & 1 & 3 & 3 & 3 & 3 & 3 & 3 & 2 \\
\hline 3 & 3 & 2 & 2 & 3 & 3 & 3 & 3 & 1 & 1 & 1 & 1 & 1 & 1 & 3 \\
\hline 4 & 2 & $\overline{8}$ & 3 & 29 & 8 & 8 & 4 & 4 & 4 & 4 & 4 & 4 & 4 & 1 \\
\hline 5 & 19 & 3 & 8 & 8 & 25 & 4 & 8 & 8 & 8 & 5 & 5 & 5 & 5 & 4 \\
\hline 6 & 4 & 19 & 25 & 25 & 4 & 25 & 24 & 24 & 5 & 7 & 7 & 7 & 7 & 5 \\
\hline 7 & 7 & 25 & 19 & 24 & 24 & 24 & 25 & 5 & 7 & 8 & 20 & 20 & 20 & 7 \\
\hline 8 & 20 & 7 & 24 & 4 & 19 & 19 & 19 & 7 & 18 & 20 & 8 & 8 & 8 & 20 \\
\hline 9 & 8 & 24 & 4 & 19 & 7 & 7 & 7 & 18 & 20 & 18 & 18 & 18 & 18 & 8 \\
\hline 10 & 5 & 4 & 7 & 7 & 20 & 20 & 20 & 20 & 24 & 19 & 19 & 19 & 19 & 18 \\
\hline 11 & 18 & 10 & 10 & 10 & 10 & 18 & 18 & 19 & 19 & 24 & 24 & 24 & 29 & 19 \\
\hline 12 & 10 & 9 & 9 & 20 & 18 & 10 & 5 & 25 & 10 & 10 & 10 & 10 & 24 & 24 \\
\hline 13 & 24 & 12 & 20 & 9 & 29 & 5 & 10 & 10 & 25 & 9 & 9 & 9 & 10 & 9 \\
\hline 14 & 12 & 20 & 12 & 12 & 9 & 9 & 9 & 9 & 9 & 12 & 12 & 12 & 9 & 10 \\
\hline 15 & 6 & 6 & 6 & 18 & 12 & 12 & 12 & 12 & 12 & 6 & 6 & 6 & 6 & 6 \\
\hline 16 & 9 & 27 & 27 & 6 & 5 & 6 & 6 & 6 & 6 & 25 & 25 & 29 & 12 & 12 \\
\hline 17 & 23 & 15 & 18 & 27 & 6 & 26 & 26 & 14 & 22 & 22 & 22 & 22 & 22 & 25 \\
\hline 18 & 22 & 5 & 26 & 5 & 27 & 27 & 27 & 22 & 14 & 14 & 14 & 25 & 25 & 22 \\
\hline 19 & 25 & 18 & 5 & 26 & 26 & 14 & 14 & 26 & 13 & 21 & 15 & 15 & 15 & 15 \\
\hline 20 & 21 & 14 & 14 & 14 & 14 & 16 & 16 & 13 & 21 & 15 & 21 & 14 & 14 & 21 \\
\hline 21 & 16 & 26 & 15 & 16 & 16 & 22 & 22. & 21 & 15 & 13 & 13 & 21 & 21 & 14 \\
\hline 22 & 14 & 23 & 23 & 23 & 23 & 23 & 13 & 15 & 26 & 23 & 29 & 13 & 13 & 13 \\
\hline 23 & 27 & 13 & 16 & 15 & 22 & 13 & 23 & 16 & 16 & 26 & 23 & 23 & 23 & 23 \\
\hline 24 & 15 & 16 & 13 & 22 & 15 & 29 & 15 & 27 & 23 & 16 & 26 & 26 & 26 & 26 \\
\hline 25 & 13 & 22 & 22 & 13 & 13 & 15 & 21 & 23 & 27 & 11 & 16 & 16 & 16 & 16 \\
\hline 26 & 28 & 21 & 21 & 21 & 21 & 21 & 29 & 11 & 11 & 27 & 11 & 11 & 11 & 11 \\
\hline 27 & 26 & 17 & 17 & 28 & 28 & 11 & 11 & 29 & 29 & 29 & 27 & 27 & 27 & 27 \\
\hline 28 & 17 & 28 & 28 & 17 & 11 & 28 & 28 & 28 & 28 & 28 & 28 & 28 & 28 & 28 \\
\hline 29 & 11 & 11 & 11 & 11 & 17 & 17 & 17 & 17 & 17 & 17 & 17 & 17 & 17 & 17 \\
\hline
\end{tabular}


Table 8b. Rankings of input vector (well) sensitivity for weight set $175 w b 7$.

\begin{tabular}{|c|c|c|c|c|c|c|c|c|c|c|c|c|c|c|}
\hline & & & & & & & arh & ion & evel & & & & & \\
\hline & -10 & -5 & $\underline{A}$ & $=$ & 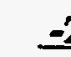 & $=1$ & -.5 & \pm .5 & \pm 1 & \pm 2 & +3 & +4 & \pm 5 & +10 \\
\hline 1 & 29 & 29 & 2 & 2 & 2 & 2 & 2 & 2 & 2 & 2 & 2 & 2 & 2 & 2 \\
\hline 2 & 1 & 1 & $I$ & 1 & 3 & 3 & 3 & 3 & 3 & 3 & 3 & 3 & 3 & 29 \\
\hline 3 & 2 & 2 & 29 & 3 & 1 & 1 & 1 & 1 & 1 & 1 & 1 & 1 & 1 & 3 \\
\hline 4 & 3 & 3 & 3 & 7 & 4 & 4 & 4 & 4 & 4 & 4 & 4 & 4 & 4 & 1 \\
\hline 5 & 8 & 7 & 7 & 4 & 7 & 7 & 7 & 5 & 5 & 5 & 5 & 5 & 5 & 4 \\
\hline 6 & 19 & 8 & 8 & 29 & 8 & 8 & 5 & 7 & 7 & 7 & 7 & 7 & 7 & 5 \\
\hline 7 & 7 & 25 & 24 & 8 & 5 & 5 & 8 & 20 & 20 & 20 & 20 & 20 & 20 & 7 \\
\hline 8 & 4 & 24 & 4 & 24 & 19 & 20 & 20 & 19 & 19 & 8 & 8 & 8 & 8 & 8 \\
\hline 9 & 25 & 10 & 10 & 10 & 20 & 19 & 19 & 8 & 8 & 19 & 19 & 19 & 19 & 20 \\
\hline 10 & 10 & 4 & 25 & 19 & 24 & 24 & 24 & 24 & 18 & 18 & 18 & 18 & 18 & 19 \\
\hline 11 & 20 & 19 & 19 & 5 & 10 & 10 & 18 & 18 & 24 & 24 & 24 & 24 & 24 & 18 \\
\hline 12 & 9 & 9 & 5 & 25 & 25 & 18 & 10 & 10 & 10 & 6 & 9 & 9 & 29 & 24 \\
\hline 13 & 12 & 5 & 20 & 20 & 18 & 25 & 25 & 6 & 6 & 9 & 12 & 12 & 9 & 9 \\
\hline 14 & 6 & 6 & 9 & 18 & 9 & 12 & 12 & 12 & 12 & 12 & 6 & 10 & 12 & 12 \\
\hline 15 & 5 & 20 & 6 & 9 & 29 & 9 & 9 & 9 & 9 & 10 & 10 & 6 & 10 & 6 \\
\hline 16 & 24 & 12 & 18 & 6 & 12 & 6 & 6 & 22 & 22 & 22 & 22 & 22 & 6 & 10 \\
\hline 17 & 18 & 18 & 12 & 12 & 6 & 22 & 22 & 25 & 25 & 21 & 21 & 29 & 22 & 22 \\
\hline 18 & 27 & 26 & 26 & 26 & 26 & 26 & 26 & 21 & 21 & 25 & 15 & 15 & 25 & 25 \\
\hline 19 & 13 & 27 & 16 & 16 & 22 & 23 & 23 & 23 & 15 & 15 & 14 & 21 & 15 & 15 \\
\hline 20 & 16 & 16 & 13 & 22 & 16 & 15 & 21 & 15 & 23 & 14 & 25 & 25 & 14 & 14 \\
\hline 21 & 15 & 13 & 27 & 15 & 15 & 16 & 15 & 14 & 14 & 13 & 13 & 14 & 21 & 21 \\
\hline 22 & 26 & 15 & 15 & 13 & 23 & 14 & 14 & 13 & 13 & 23 & 23 & 13 & 13 & 13 \\
\hline 23 & 14 & 14 & 22 & 23 & 13 & 21 & 13 & 26 & 26 & 16 & 16 & 23 & 23 & 23 \\
\hline 24 & 23 & 22 & 14 & 14 & 14 & 13 & 16 & 16 & 16 & 26 & 29 & 26 & 26 & 26 \\
\hline 25 & 22 & 23 & 23 & 27 & 21 & 29 & 27 & 27 & 27 & 11 & 26 & 16 & 16 & 16 \\
\hline 26 & 21 & 28 & 28 & 21 & 27 & 27 & 29 & 11 & 11 & 27 & 11 & 11 & 11 & 27 \\
\hline 27 & 28 & 11 & 21 & 28 & 28 & 11 & 11 & 29 & 29 & 29 & 27 & 27 & 27 & 11 \\
\hline 28 & 17 & 21 & 11 & 11 & 11 & 28 & 28 & 28 & 28 & 17 & 17 & 17 & 17 & 28 \\
\hline 29 & 11 & 17 & 17 & 17 & 17 & 17 & 17 & 17 & 17 & 28 & 28 & 28 & 28 & 17 \\
\hline
\end{tabular}


Table 8c. Rankings of input vector (well) sensitivity for weight set 175 wa9.

\begin{tabular}{|c|c|c|c|c|c|c|c|c|c|c|c|c|c|c|}
\hline & \multicolumn{14}{|c|}{ Perturbation Level } \\
\hline \multicolumn{2}{|c|}{ Rank $=10$} & $\mathbf{5}$ & -4 & $=3$ & $=$ & -1 &. .5 & \pm .5 & \pm 1 & \pm 2 & +3 & +4 & \pm 5 & +10 \\
\hline$\frac{1}{2}$ & $\begin{array}{l}2 \\
3\end{array}$ & $\begin{array}{l}4 \\
3\end{array}$ & $\frac{1}{3}$ & $\begin{array}{l}1 \\
3\end{array}$ & $\begin{array}{l}1 \\
3\end{array}$ & $\begin{array}{l}3 \\
1\end{array}$ & $\begin{array}{r}3 \\
19\end{array}$ & $\begin{array}{r}19 \\
3\end{array}$ & $\begin{array}{r}3 \\
19\end{array}$ & $\begin{array}{l}3 \\
2\end{array}$ & $\begin{array}{l}3 \\
2\end{array}$ & $\begin{array}{l}2 \\
3\end{array}$ & $\begin{array}{l}2 \\
3\end{array}$ & $\begin{array}{l}2 \\
3\end{array}$ \\
\hline 3 & 4 & 2 & 2 & 2 & 2 & 19 & 2 & 2 & 2 & 8 & 1 & $I$ & 1 & $\begin{array}{l}3 \\
4\end{array}$ \\
\hline 4 & 5 & 1 & 4 & 4 & $1 \overline{9}$ & 2 & 1 & 8 & 8 & 4 & 4 & 4 & $\overline{4}$ & $i$ \\
\hline 5 & 1 & 5 & 7 & 8 & 4 & 4 & 24 & 4 & 4 & 1 & 8 & 5 & 5 & 5 \\
\hline 6 & 7 & 7 & 19 & 19 & 8 & 8 & 4 & 24 & 1 & $1 \overline{9}$ & 5 & 7 & 7 & 7 \\
\hline 7 & 20 & 19 & 20 & 7 & 10 & 24 & 8 & 1 & 24 & 20 & 7 & 8 & 8 & 8 \\
\hline 8 & 18 & 20 & 5 & 29 & 20 & 27 & 25 & 25 & 20 & 18 & 19 & 20 & 20 & 20 \\
\hline 9 & 19 & 18 & 8 & 20 & 7 & 25 & 27 & 27 & 18 & 7 & 20 & 19 & 19 & 18 \\
\hline 10 & 8 & 8 & 29 & 6 & 27 & 10 & 10 & 10 & 25 & 24 & 18 & 18 & 18 & 19 \\
\hline 11 & 24 & 6 & 6 & 10 & 24 & 20 & 20 & 20 & 10 & 5 & 24 & 24 & 24 & 29 \\
\hline 12 & 12 & 12 & 18 & 5 & 25 & 7 & 7 & 22 & 7 & 10 & 10 & 10 & 10 & 24 \\
\hline 13 & 9 & 10 & 12 & 12 & 29 & 12 & 12 & 18 & 22 & 12 & 12 & 9 & 9 & 9 \\
\hline 14 & 22 & 29 & 10 & $9^{\prime}$ & 12 & 9 & 9 & 7 & 27 & 25 & 6 & 12 & 12 & 12 \\
\hline 15 & 6 & 9 & 9 & 18 & 6 & 18 & 18 & 12 & 12 & 9 & 9 & 6 & 6 & 10 \\
\hline 16 & 10 & 24 & 24 & 24 & 18 & 6 & 22 & 9 & 9 & 6 & 25 & 25 & 22 & 6 \\
\hline 17 & 21 & 22 & 15 & 15 & 9 & 23 & 6 & 23 & 21 & 22 & 22 & 22 & 25 & 22 \\
\hline 18 & 14 & 14 & 14 & 23 & 5 & 26 & 23 & 21 & 23 & 21 & 13 & 13 & 13 & 25 \\
\hline 19 & 13 & 15 & 13 & 16 & 16 & 16 & 26 & 6 & 5 & 13 & 14 & 14 & 14 & 15 \\
\hline 20 & 15 & 13 & 23 & 25 & 23 & 5 & 16 & 14 & 6 & 16 & 21 & 15 & 15 & 14 \\
\hline 21 & 23 & 23 & 22 & 14 & 26 & 14 & 21 & 5 & 14 & 14 & 15 & 21 & 21 & 21 \\
\hline 22 & 25 & 16 & 21 & 21 & 15 & 22 & 14 & 15 & 16 & 23 & 16 & 16 & 23 & 13 \\
\hline 23 & 16 & 21 & 16 & 27 & 14 & 15 & 15 & 13 & 13 & 27 & 23 & 23 & 16 & 23 \\
\hline 24 & 26 & 25 & 25 & 13 & 21 & 21 & 5 & 16 & 15 & 15 & 26 & 26 & 26 & 26 \\
\hline 25 & 29 & 26 & 26 & 26 & 22 & 29 & 13 & 26 & 26 & 26 & 27 & 11 & 11 & 16 \\
\hline 26 & 11 & 11 & 27 & 17 & 17 & 13 & 29 & 11 & 11 & 11 & 11 & 27 & 27 & 11 \\
\hline 27 & 27 & 27 & 11 & 22 & 13 & 28 & 28 & 28 & 28 & 17 & 17 & 17 & 29 & 27 \\
\hline 28 & 28 & 17 & 17 & 11 & 28 & 11 & 11 & 29 & 17 & 28 & 28 & 29 & 17 & 28 \\
\hline 29 & 17 & 28 & 28 & 28 & 11 & 17 & 17 & 17 & 29 & 29 & 29 & 28 & 28 & 17 \\
\hline
\end{tabular}


Table 8d. Rankings of input vector (well) sensitivity for weight set $175 w b 9$.

\begin{tabular}{|c|c|c|c|c|c|c|c|c|c|c|c|c|c|c|}
\hline \multicolumn{15}{|c|}{ Perturbation Level } \\
\hline \multicolumn{2}{|c|}{ Rank $=10$} & $\underline{-5}$ & $\underline{4}$ & $\underline{-3}$ & -2 & -1 & -.5 & \pm .5 & \pm 1 & \pm 2 & +3 & +4 & \pm 5 & +10 \\
\hline 1 & 2 & 2 & 1 & 1 & 1 & 1 & 2 & 2 & 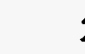 & 2 & 2 & 2 & 2 & 2 \\
\hline 2 & 4 & 3 & 2 & 2 & 2 & 2 & 1 & 3 & 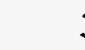 & 3 & 3 & 3 & 3 & 3 \\
\hline 3 & 3 & 4 & 3 & 3 & 3 & 3 & 3 & 1 & & 1 & 20 & 20 & 4 & 4 \\
\hline 4 & 1 & 1 & 4 & 4 & 7 & 8 & 24 & 24 & 2 & 20 & 1 & 1 & 1 & 1 \\
\hline 5 & 5 & 5 & 5 & 10 & 8 & 7 & 8 & 8 & & 19 & 19 & 19 & 20 & 5 \\
\hline 6 & 7 & 7 & 7 & 5 & 10 & 24 & 7 & 7 & & 7 & 18 & 4 & 5 & 7 \\
\hline 7 & 20 & 18 & 10 & 7 & 4 & 19 & 19 & 19 & 1 & 24 & 7 & 5 & 19 & 20 \\
\hline 8 & 18 & 20 & 20 & 8 & 19 & 10 & 4 & 4 & & 18 & 4 & 18 & 7 & 18 \\
\hline 9 & 19 & 19 & 18 & 19 & 5 & 4 & 10 & 18 & 1 & 8 & 5 & 7 & 18 & 19 \\
\hline 10 & 8 & 8 & 19 & 18 & 6 & 6 & 18 & 20 & 2 & 4 & 22 & 8 & 8 & 8 \\
\hline 11 & 24 & 10 & 8 & 20 & 18 & 18 & 20 & 25 & & 22 & 8 & 22 & 22 & 24 \\
\hline 12 & 9 & 9 & 9 & 6 & 20 & 20 & 25 & 5 & 2 & 5 & 24 & 24 & 12 & 9 \\
\hline 13 & 12 & 12 & 12 & 9 & 24 & 5 & 6 & 10 & 2 & 23 & 21 & 21 & 24 & 12 \\
\hline 14 & 22 & 24 & 6 & 12 & 12 & 25 & 5 & 26 & 2. & 21 & 23 & 12 & 9 & 29 \\
\hline 15 & 10 & 6 & 15 & 16 & 15 & 12 & 12 & 22 & 2 & 12 & 12 & 23 & $2 I$ & 22 \\
\hline 16 & 6 & 15 & 24 & 15 & 9 & 21 & 26 & 23 & 1 & 10 & 10 & 10 & 10 & 10 \\
\hline 17 & 21 & 14 & 14 & 14 & 16 & 23 & 23 & 12 & 1 & 25 & 6 & 9 & 6 & 6 \\
\hline 18 & 15 & 22 & 16 & 24 & 14 & 15 & 21 & 6 & 2 & 26 & 9 & 6 & 23 & 21 \\
\hline 19 & 14 & 13 & 13 & 29 & 21 & 16 & 16 & 21 & & 6 & 25 & 14 & 14 & 15 \\
\hline 20 & 13 & 21 & 29 & 11 & 23 & 26 & 27 & 27 & 2 & 9 & 14 & 15 & 15 & 14 \\
\hline 21 & 23 & 16 & 11 & 13 & 27 & 27 & 15 & 16 & 1 & 27 & 15 & 13 & 13 & 13 \\
\hline 22 & 25 & 11 & 22 & 21 & 25 & 14 & 22 & 14 & & 14 & 26 & 25 & 25 & 23 \\
\hline 23 & 29 & 25 & 21 & 27 & 29 & 9 & 14 & 9 & 1 & 16 & 13 & 16 & 16 & 25 \\
\hline 24 & 26 & 23 & 23 & 23 & 26 & 22 & 9 & 15 & 1 & 15 & 27 & 26 & 29 & 26 \\
\hline 25 & 16 & 29 & 27 & 22 & 13 & 28 & 28 & 28 & 2 & 13 & 16 & 27 & 26 & 16 \\
\hline 26 & 11 & 26 & 25 & 17 & 11 & 13 & 13 & 13 & 1 & 28 & 28 & 29 & 11 & 11 \\
\hline 27 & 27 & 27 & 17 & 25 & 22 & 29 & 11 & 29 & 2 & 29 & 29 & 11 & 27 & 27 \\
\hline 28 & 28 & 17 & 26 & 26 & 17 & 11 & 29 & 11 & 1 & 11 & 11 & 28 & 28 & 28 \\
\hline 29 & 17 & 28 & 28 & 28 & 28 & 17 & 17 & 17 & 1 & 17 & 17 & 17 & 17 & 17 \\
\hline
\end{tabular}




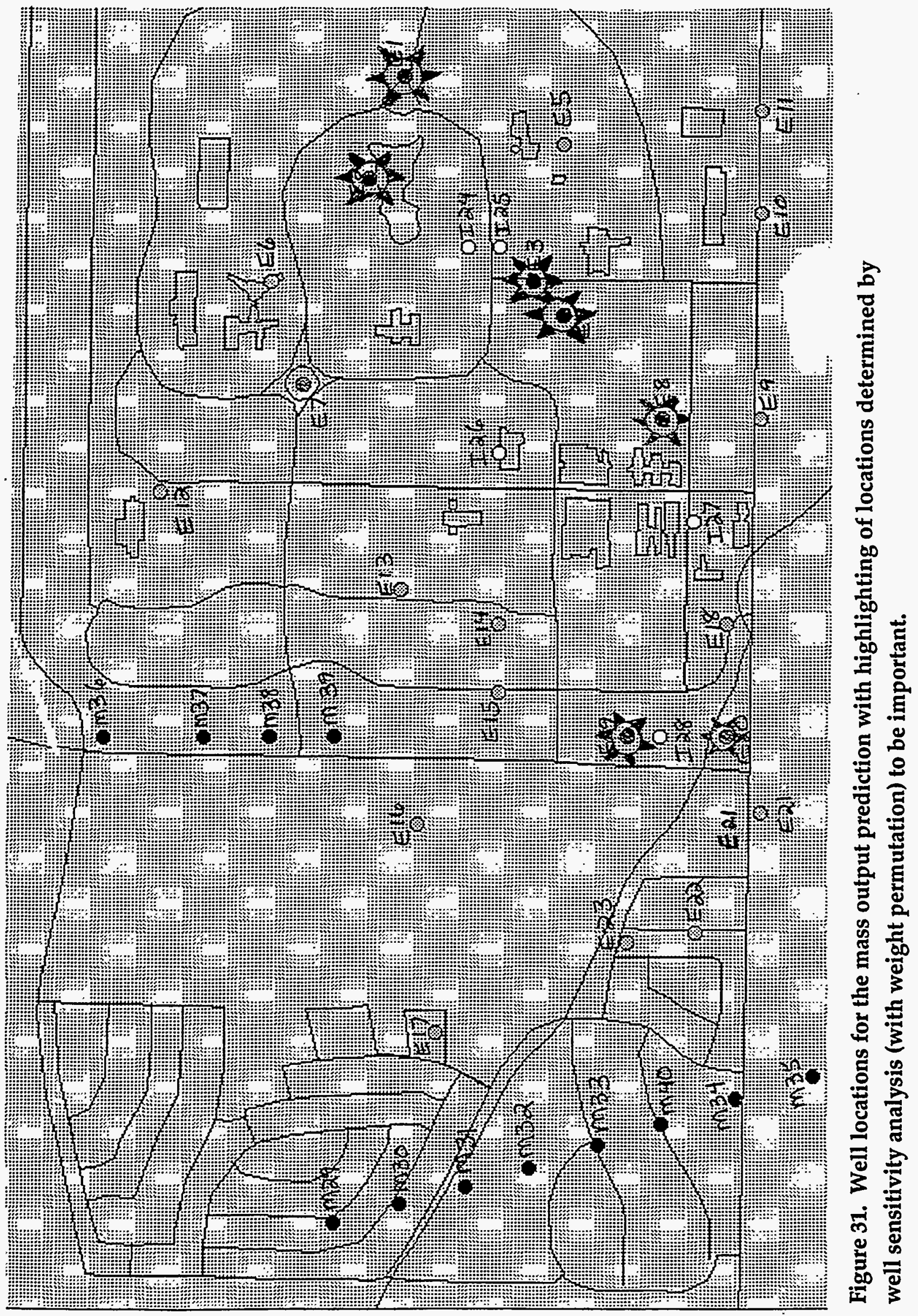




\subsection{Multiple Objectives}

The same set of 225 pumping pattern realizations was run on a more complex flow and transport code in order to develop training patterns for multiple objectives, including cost of remediation and successful containment of the plume within its current boundaries. The 2-D hybrid finite-element/finite-difference transport code, SUTRA, (Voss, 1984) was used with similar boundary conditions and source/sink terms as the PLANET runs (Figure 32). The general SUTRA equation solved here is based upon the governing equations for confined areal ground water flow and areal solute transport (See Eqs. 15 through 18).

First a network was trained to predict two outputs: 1) success of remediation at keeping concentrations in a fence of monitor wells at the plume boundary from exceeding $10 \mathrm{ppb}$ and 2) cost of remediation. The cost function included calculations of facilities, piping, operating, and water treatment costs. The total costs were considered to result from capital and operating costs:

Objective: minimize $C^{T}=\Sigma\left(C^{C A P I T A L}+\right.$ COPER\&MAINT $)$

where:

$C^{T}=$ total cost of groundwater remediation

CCAPITAL $=\Sigma C^{\text {TFS }}$

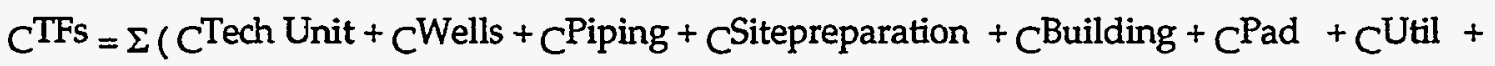

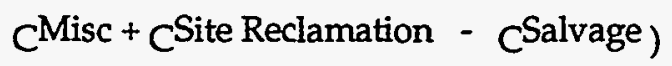

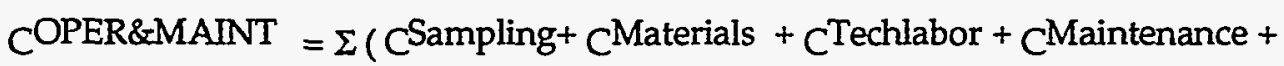

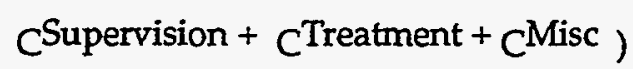

The cost of pumping and injection wells was differentiated. The cost of treatment of groundwater pumped to the surface was averaged between the different treatment protocols to be approximately one cent per every four liters. 


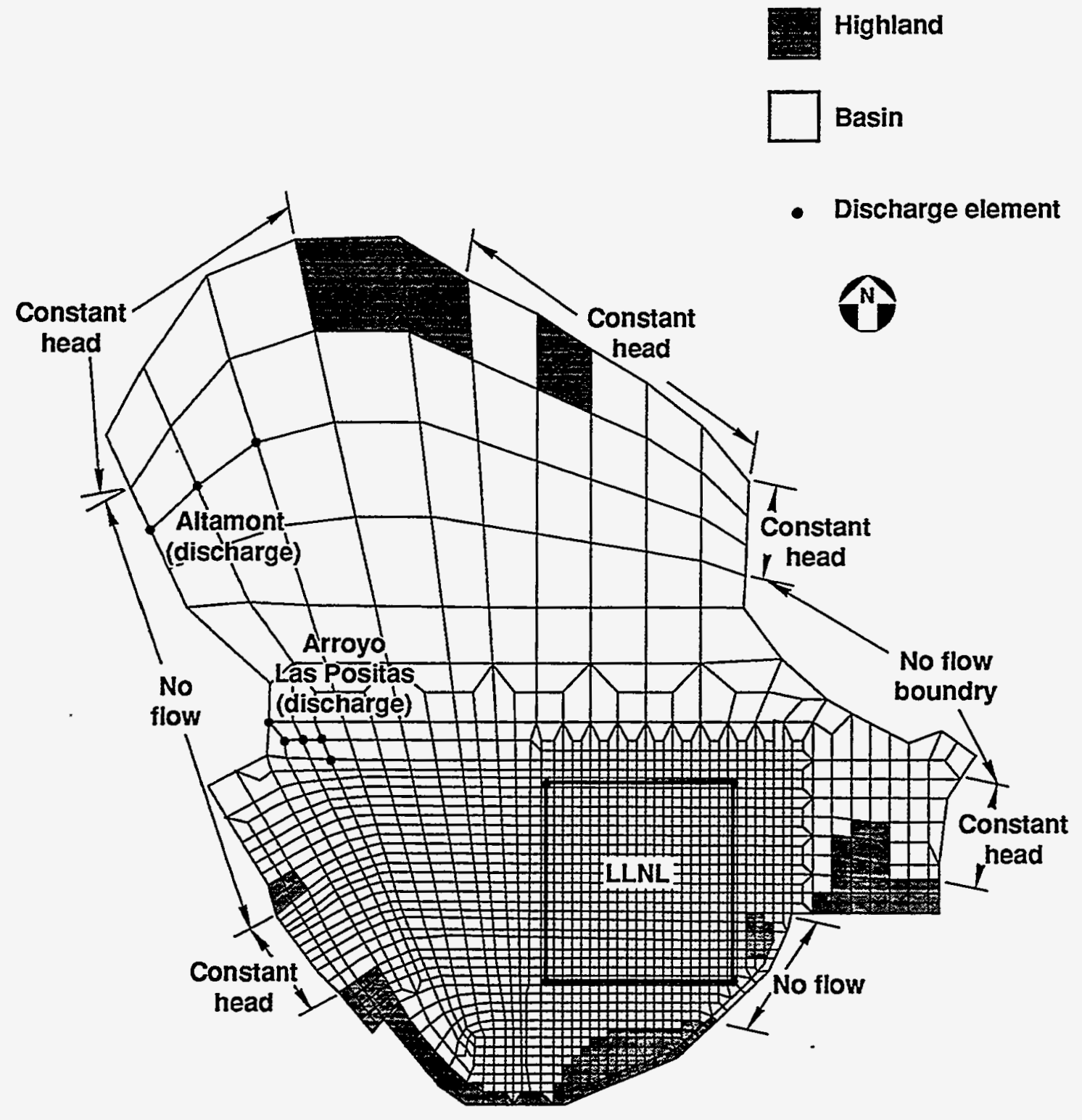

Figure 32. Numerical grid and boundaries for SUTRA flow and transport simulation at LLNL. 


\subsubsection{Network Training}

The performance of a network trained for two outputs was poorer than if two networks were trained separately, each on one output. This can be seen in the generalization performance of the network trained to predict two outputs including cost (Figure 33) versus the generalization performance of a network trained to only the cost function (Figure 34). Note that these results are from using the same patterns of training and testing. This suggests significantly better performance can be obtained by using multiple networks to predict multiple outputs. One may improve the performance with more training runs. However, this multiple network approach (Figure 35) is a strategy to minimize the number of flow and transport simulations one needs to solve the management problem.

\subsubsection{Optimization}

The unremediated transport over the next 40 years will result in further contamination of groundwater under residential areas to the west of LLNL (Figure 36). Having all 28 wells on will prevent this further migration of the plume as well as reduce the contamination remaining on the site (Figure 37). However, one can see as much as 35 million dollars may be saved by finding realizations of pumping such as that shown in Figure 38 which also halt migration but involve fewer pumping wells. This latter pumping realization was found during only 8 generations of GA directed search. The fitness parameter for the GA generations was a linear combination of three objectives (Table 9), 1) whether the plume had been constrained behind selected monitor wells (value of 1 or 0$), 2$ ) one minus the normalized and scaled (.1 to .9) value of time to clean up all concentrations to less than $300 \mathrm{ppb}$, and 3) one minus the normalized and scaled (again .1 to .9) value of cost of remediation over the 40 years. 
옹

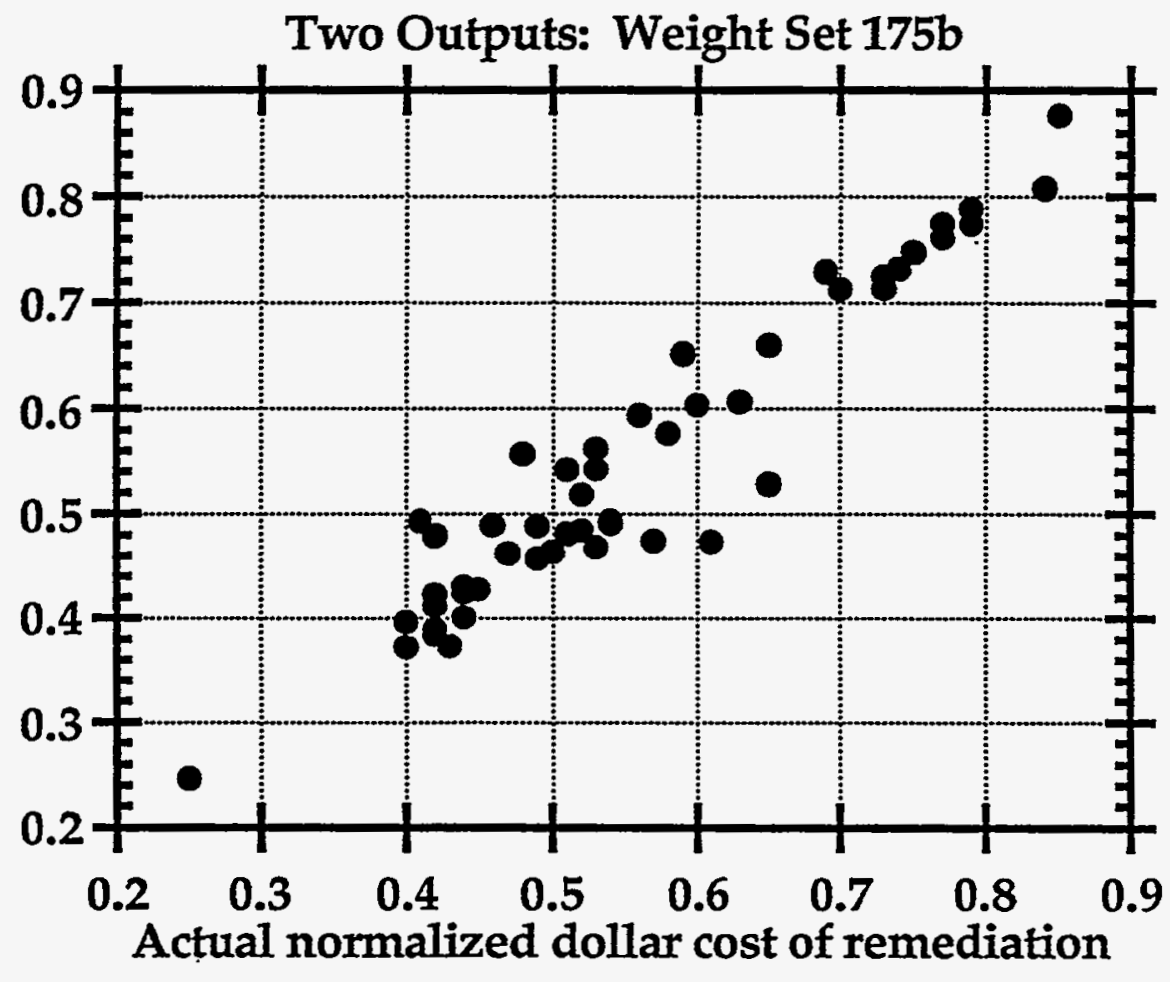

Figure 33. Predicted versus actual cost of remediation for the network trained to two outputs. Cost is normalized. 


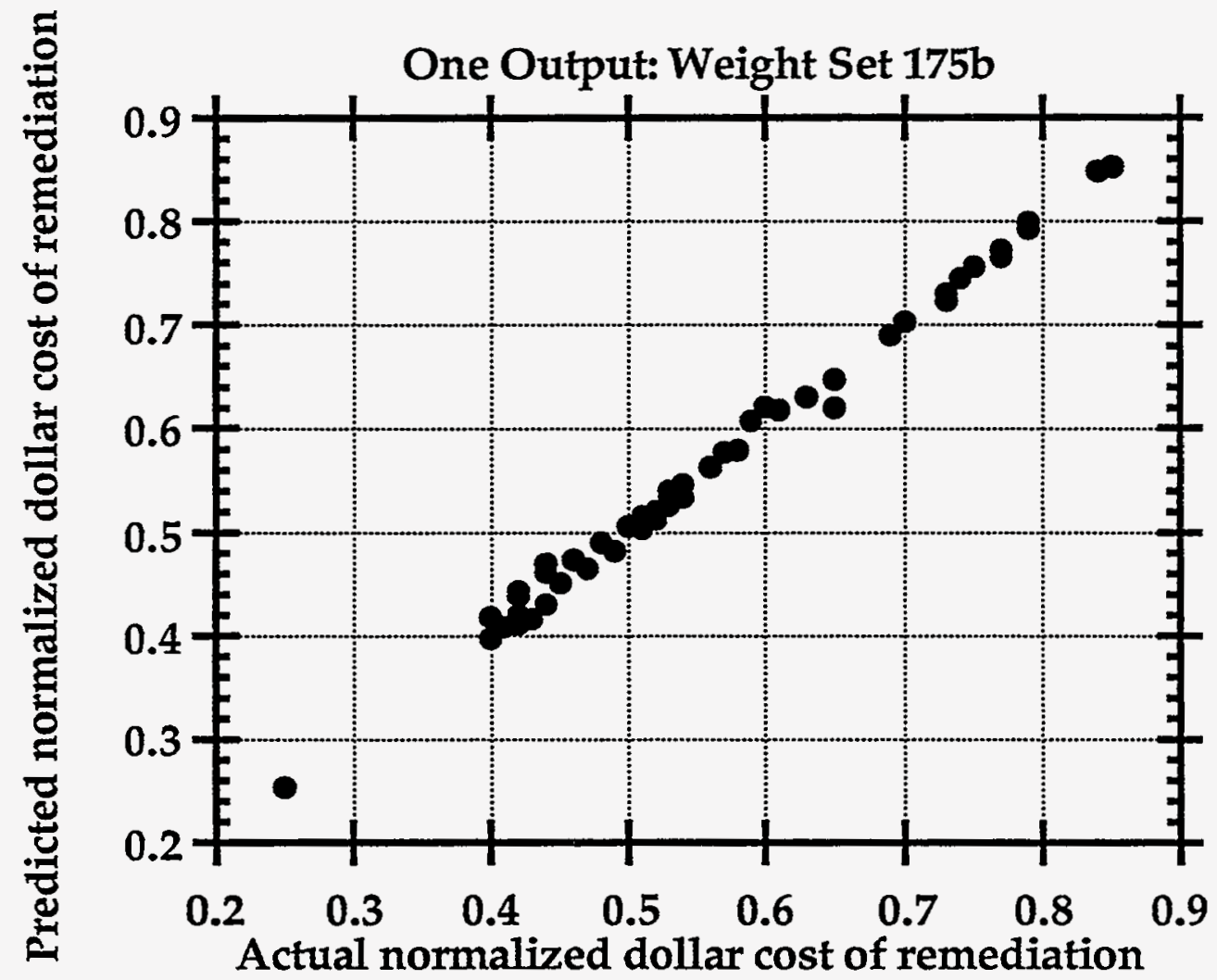

Figure 34. Predicted versus actual cost of remediation for the network trained to only one output. Cost is normalized. 


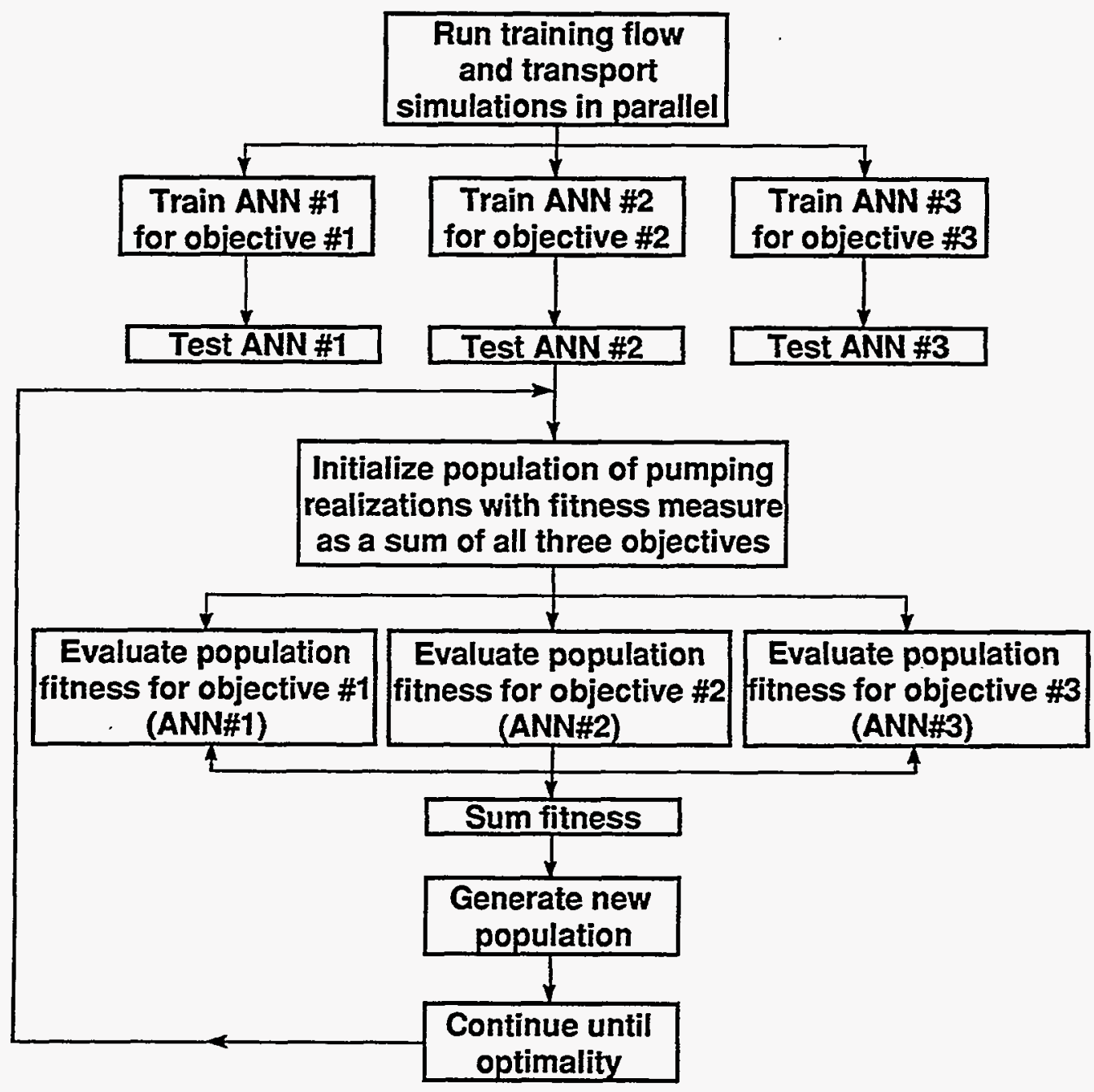

Figure 35. Flowchart of three ANNs trained for three-objective remediation. 


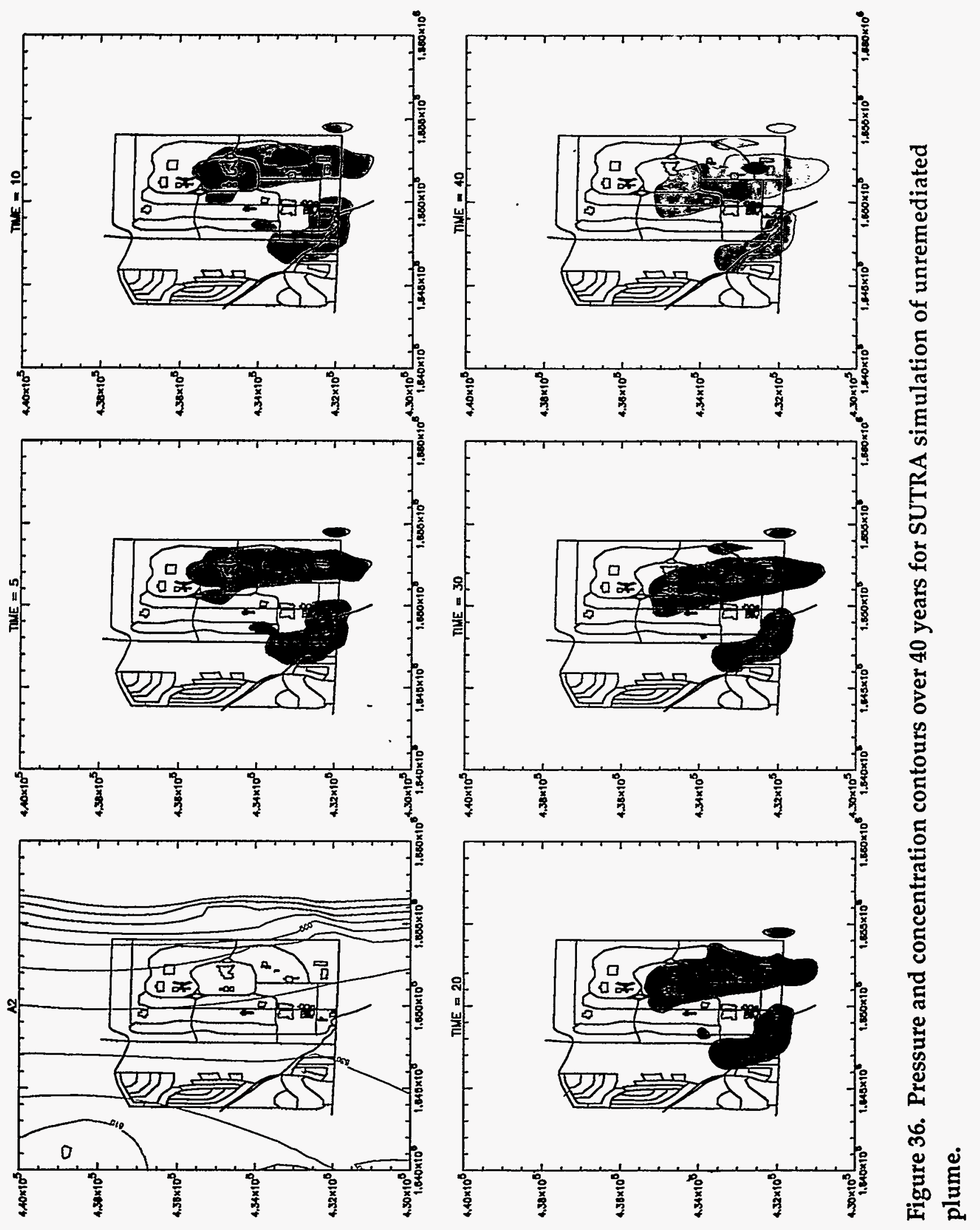



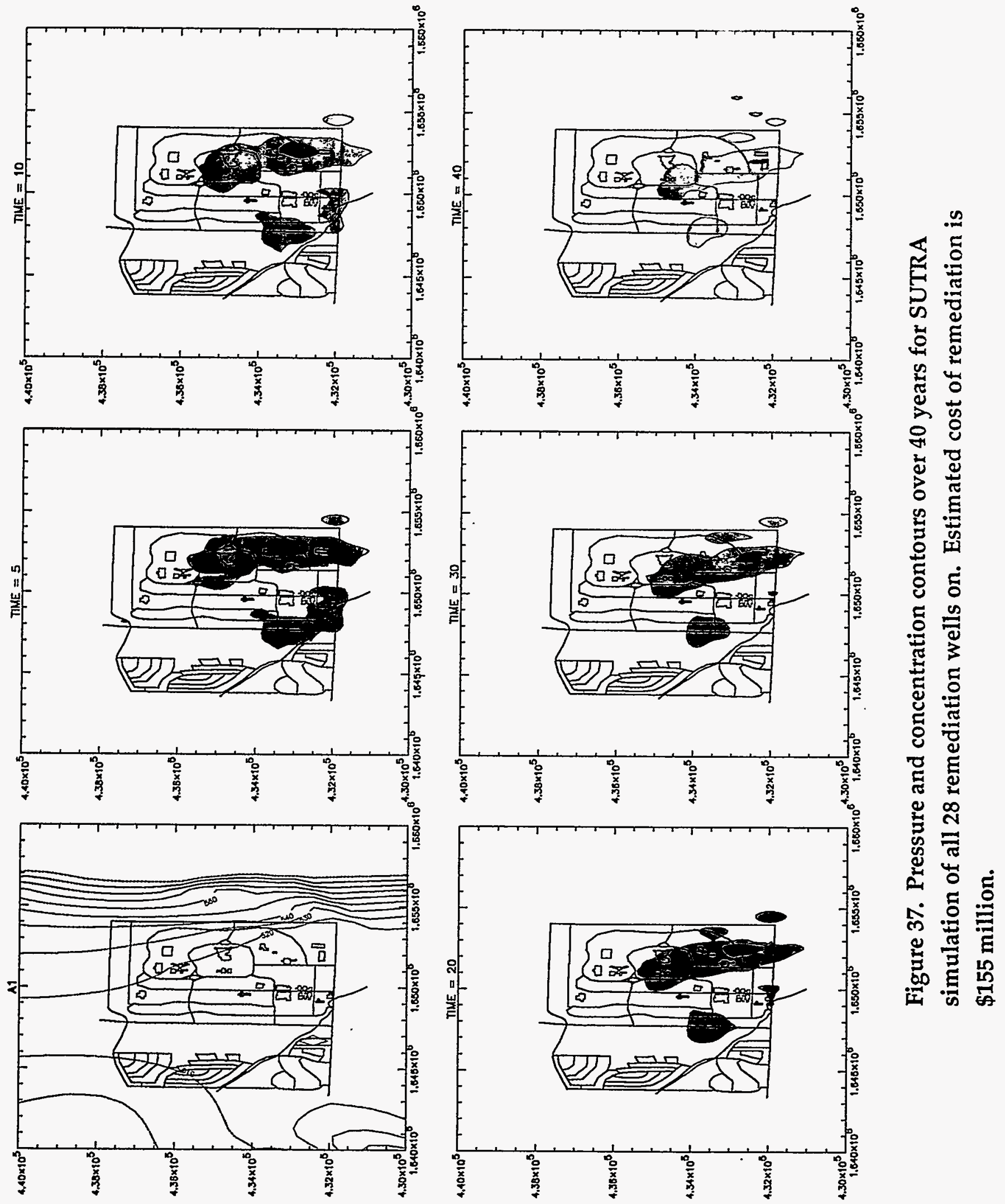


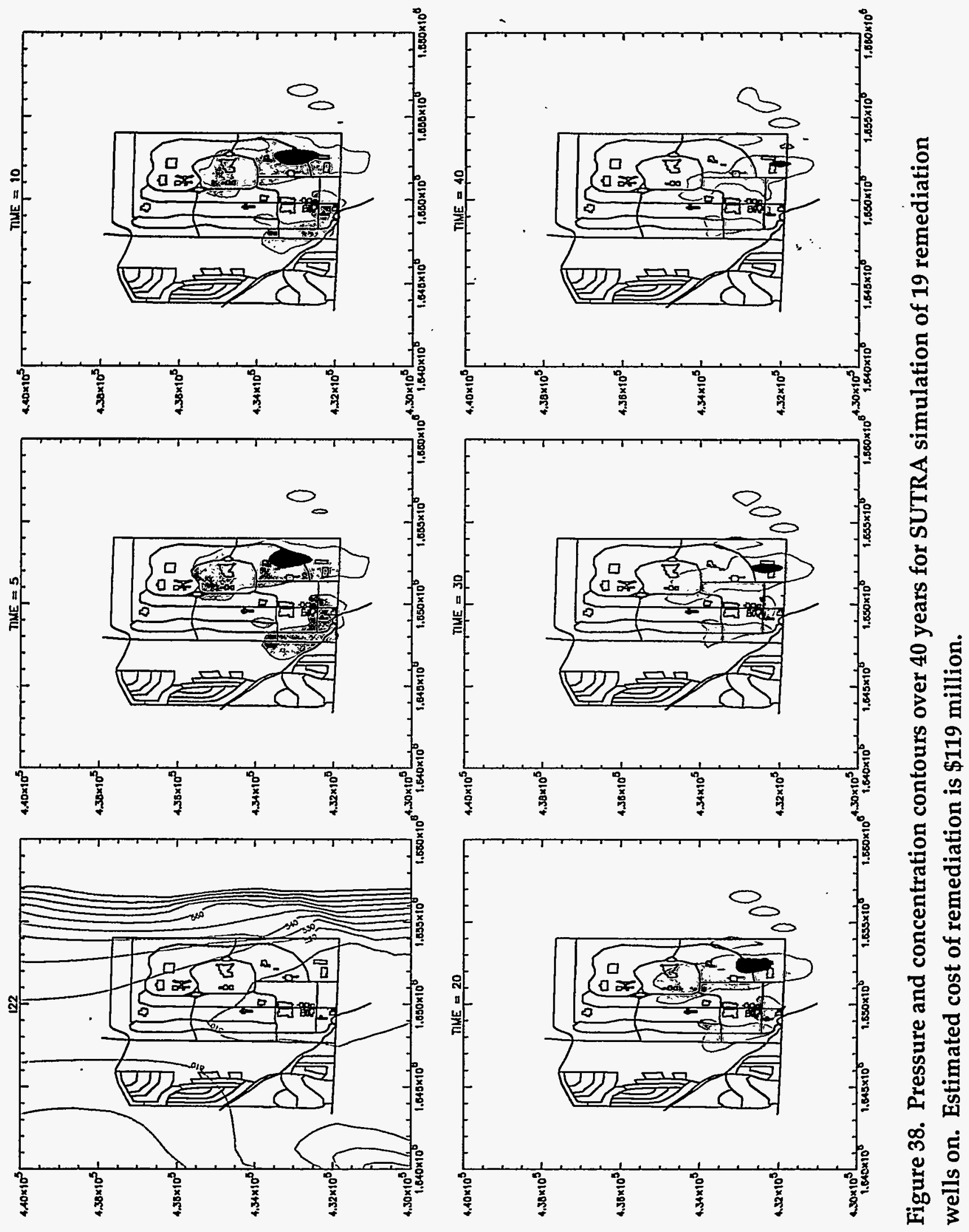


Table 9: Transfer of information from one generation to the next according to the genetic algorithm for the multiple objectives field-scale application.

a) a current generation.

\begin{tabular}{|c|c|c|c|c|c|c|c|}
\hline No. & Pattern of Pumping & $\begin{array}{l}\text { Pumping } \\
\text { Param. }\end{array}$ & $\begin{array}{l}\text { Prob. of } \\
\text { Selection }\end{array}$ & $\begin{array}{l}\text { Reg } \\
\text { Const }\end{array}$ & $\begin{array}{l}\text { Time } \\
\text { for }< \\
300 \mathrm{ppb}\end{array}$ & Cost & $\begin{array}{l}\text { Measure } \\
\text { of } \\
\text { Fitness@ }\end{array}$ \\
\hline $\mathbf{I}$ & 1111111110011000011111111 & 100 & 0.024 & 1 & $\overline{0.296}$ & $\overline{0.646}$ & 1.943 \\
\hline 2 & 1111111110000000000000000 & 000 & 0.020 & 1 & 0.306 & 0.327 & 1.632 \\
\hline 141 & 1011100111100111010000100 & 100 & 0.019 & 1 & 0.092 & 0.426 & 1.518 \\
\hline 142 & 1101011011010100010111111 & 100 & .390 .020 & 0 & 0.959 & 0.663 & 1.622 \\
\hline \multicolumn{8}{|c|}{ Total Fitness of Population } \\
\hline
\end{tabular}

b) mating pool after reproduction,

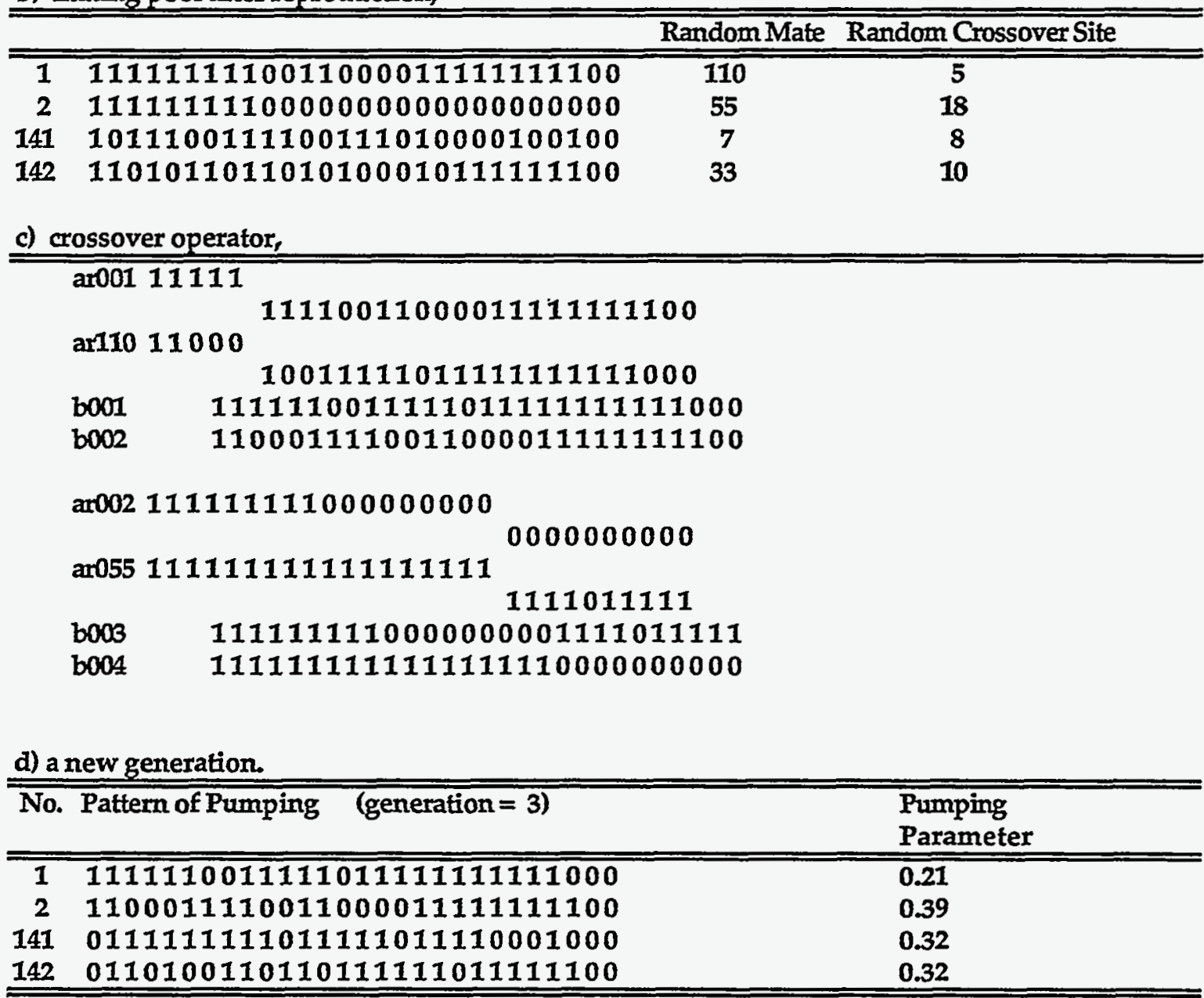

(a) The measure of fitness was a linear combination of the three objectives, i.e., measure of fitness $=($ was the regulatory constraint met, either 1 or 0$)+(1$-normalized time to clean up the site to $<300 \mathrm{ppb})+(1$-the normalized cost of remediation). 


\subsection{Issues and Analogies}

Several issues are touched on briefly during previous chapters of this dissertation that deserve more elaboration. The issues gathered together in this section are 1) amount of data required for appropriate mapping of an problem, 2) number of hidden layer nodes, 3) improvement of learning rates, 4) and generalization ablilities. These issues involve how one most efficiently finds the best mapping of a problem and how the network captures that mapping. The motivation of this section is to give the reader a more developed framework for understanding how ANNs are designed and how to realize the potential of ANN applications. The discussion is made in the context of the backpropagation learning algorithm, as backpropagation was used in this work. As these issues are still regarded as areas of continuing ANN research, this discussion is only designed as a stepping-stone for those interested in ANN applications.

In the course of this discussion analogies are presented with terminology and concepts which may be more familiar to the groundwater hydrologist, applied earth scientist, or others interested in remediation. The reader is cautioned to use these analogies as generalities. Every analogy will reach a level at which it will no longer apply. It is hoped that these analogies will not be overused and thus, limit the reader in trying to describe something new solely with terminology that is familiar rather than with new terminology which may more eloquently describe the processes.

Several theoretical studies have independently found that a three-layer ANN trained with the backpropagation algortihm can approximate a diverse range of functions (Irie and Miyake, 1988, Moore and Poggio, 1988; White, 1988). Readers are also refered to Kolmogorov's 
theoretical development (Kolmogrov, 1957) which proves that a three-layer ANN can perform any continuous nonlinear mapping. The overall concensus of this work is that if a mapping exists the backpropagation learning algorithm can be used to train an ANN to find it to any desired degree of accuracy. The work does not state how to find this mapping (Simpson, 1990).

\subsection{Appropriate Number of Training Patterns}

One important issue in finding an appropriate mapping is the amount of data required for this mapping. In the context of an ANN application, one asks how many training and testing patterns, or examples, should one have and how should they be chosen so that the network can learn enough information over the vector space or solution space of interest to appropriately map the problem. There is no single definition of how many training and testing patterns are appropriate for a given problem, because to a large extent this depends on the range of possible patterns and the distribution of the training and testing chosen from the possible patterns. As discussed earlier, one empirical rule of thumb is that when the examples are relatively uncorrelated, a network should be trained on at least as many examples as the number of network weights. Intuitively, the larger and more complex the problem, the more training examples will be necessary for the network to learn the vital elements of the problem. Maren et. al. (1990) quote Stubbs in personnal communication as suggesting a number of training patterns that is at least five times the number of nodes in the network. It is also suggested by seasoned researchers that the number of training patterns be ten times the number of network weights (Rumelhart, personal communication). Note that while the applications in this dissertation had only slightly more training examples than the number of network weights, the generalization performance was judged to be good. A network's calculated performance is also somewhat dependent on how stringent the researcher decides to be on the margin of error between the predicted and actual output.

Analogies to training and testing which may be more familiar to a reader from an earth sciences background are 1) the analogy of ANN training and ANN learning to calibration of 
models or equipment, and 2) the analogy of testing of an ANN to verification of models or equipment.

Two other questions are how does one choose the training patterns, and is overfitting to the training patterns a problem. Intuitively, the choice of training patterns should reflect the overall problem as best as possible. In other words, make sure that the training data cover the entire expected input variations. Another way to think of this problem is: it is more reasonable to ask the ANN to interpolate than to extrapolate. Randomly generated training patterns can be thought of as describing the overall solution space, although perhaps sparsely if the number of training patterns is small relative to the total number of possilbe patterns.

The second question, i.e., is overfitting to the training patterns a problem, was discussed in Sections 2.0 and 3.0 in relation to network design, i.e., implementing weight elimination to penalize network complexity. We can also discuss this issue in relation to analogies of ANN to regression. Many people seek to compare regression or a curve-fitting process to the ANN's effort to find the appropriate weights which will enable the best match of its predicted answers with the actual. Describing the ANN procedure as curve-fitting can be misleading to many who associate this with a preconceived model being fit to data points. An analogy can be made to a non-parametric fitting where no preconcieved model is used; however, one must be careful not to overuse or be limited by this analogy. Perhaps it is better to refer to the ANN's ability to capture a very irregular solution space by combining many highly nonlinear functions. If we consider the concept of curve-fitting in a very broad context, curve fitting is neither simple nor completely understood. Three aspects which make curve-fitting quite difficult are 1) nonuniform sampling, 2) multi-dimensionality, and 3) nonlinearity. In the analogy to ANN, the nonuniform sampling is analogous to difficulties in choosing the number and type of training patterns. The multi-dimensionality can be thought of as analogous to how many input nodes are in a network. And the nonlinearity can be analogous to the number of hidden nodes, layers, and the nonlinear threshold function to transform input to the other network nodes. 
One of the simplest examples of a poor sampling of data, and thus a poor mapping would be if one trained an ANN on patterns which were meant to be random and uncorrelated, yet the ANN perceived a correlation. There is a great deal in the literature concerning appropriate sampling in the areas of statistics, signal-processing, regression analysis, etc. The reader is also refered to the relevant ANN work of Tishby et al. (1989).

While this discussion emphasized back-propagation networks, the issue of overfitting brings up a very accurate analogy to training on the noise in the data. This is a subject of great interest in signal-processing and other ANN learning alogirthms. Grossberg (1976) spoke of the limitation of his Additive Grossberg learning algorithm, which encoded signal and noise with equal importance, as the noise-saturation dilemma. In a pure signal application this dilemma may be addressed by the addition of an automatic gain control over the input signal that decreases the relative importance of noise. However, in many real world problems the noise in the data is not so easily dealt with.

\subsection{Appropriate Number of Hidden Layer Nodes}

Several researchers have proposed heuristics as a method to delete unnecessary hidden layer nodes from the network (Sietsma and Dow,1988). Ash (1988) presented a technique, dynamic node creation, that adds hidden layer nodes as they are determined to be necessary. The method of weight elimination, as discussed in Sections 2.0 and 3.0, is also a way to penalizes network, and in effect diminish the weights to the minimum necessary. Thus, even if the nodes are still programmed into the network architecture, they will practically not be a burden to the network because their weights are so close to zero.

\subsection{Improvement of Learning Rates}

The original backpropagation learning algorithm has been altered, as discussed in Section 2.0, to eliminate the guesswork of determining an optimal learning rate in the backpropagation learning algorithm. The Newton method has been used by many researchers (Sutton, 1986; Parker, 1987). Many other methods and heuristics have been introduced to 
decrease the training time of the backpropagation algorithm. A momentum term, a component of the the last weight change, has been used (Rumelhart et al, 1986). Much of the research today focuses on employing robust optimization techniques such as the conjugate gradient method Johnasson et al., 1990) to speed convergence.

\subsection{Generalization Abilities}

An issue of significant interest, particularly in real-world problems, is the ability of an ANN to generalize and understand the problem from the training data (Baum, 1986; Denker, 1987; Gallinari, et al., 1987; Psaltis and Neifield, 1988; Musari, et al., 1988, Smieja, 1988).

Generalization refers here to the ability of the ANN to identify the key relationships between the input training vectors and downplay irrelevant relationships.

One analogy which comes up in the discussion of generalization and how the network actually encodes the vital components of the nonlinear problem, is the similarity of the maturing weights with principal component analysis of statistics. One might think of the weights which the network has converged to as a refining of the problem down to these major components, particularly if weight-elimination procedures are implemented.

There is a general increase in generalization ability of a network with increasing number of examples for the same network complexity, i.e., number of network connections (Figure 39). We can think of a general area (shaded region in Figure 39) which comprises a reasonable mapping of a problem by an ANN. A greater generalization may be possible of the problemin this acceptable region (G2); however, it may require more training examples (relative to G1).

Poggio and Girosi (1990) approached the problem of generalization from the perspective of approximation theory, and constructed a theoretical framework with which to examine network learning. Pointing out the parallels in learning with classical approximation techniques, such as generalized splines and regularization theory, they present a class of three-layer networks, which they call regularization networks. Regularization networks, which are closely 


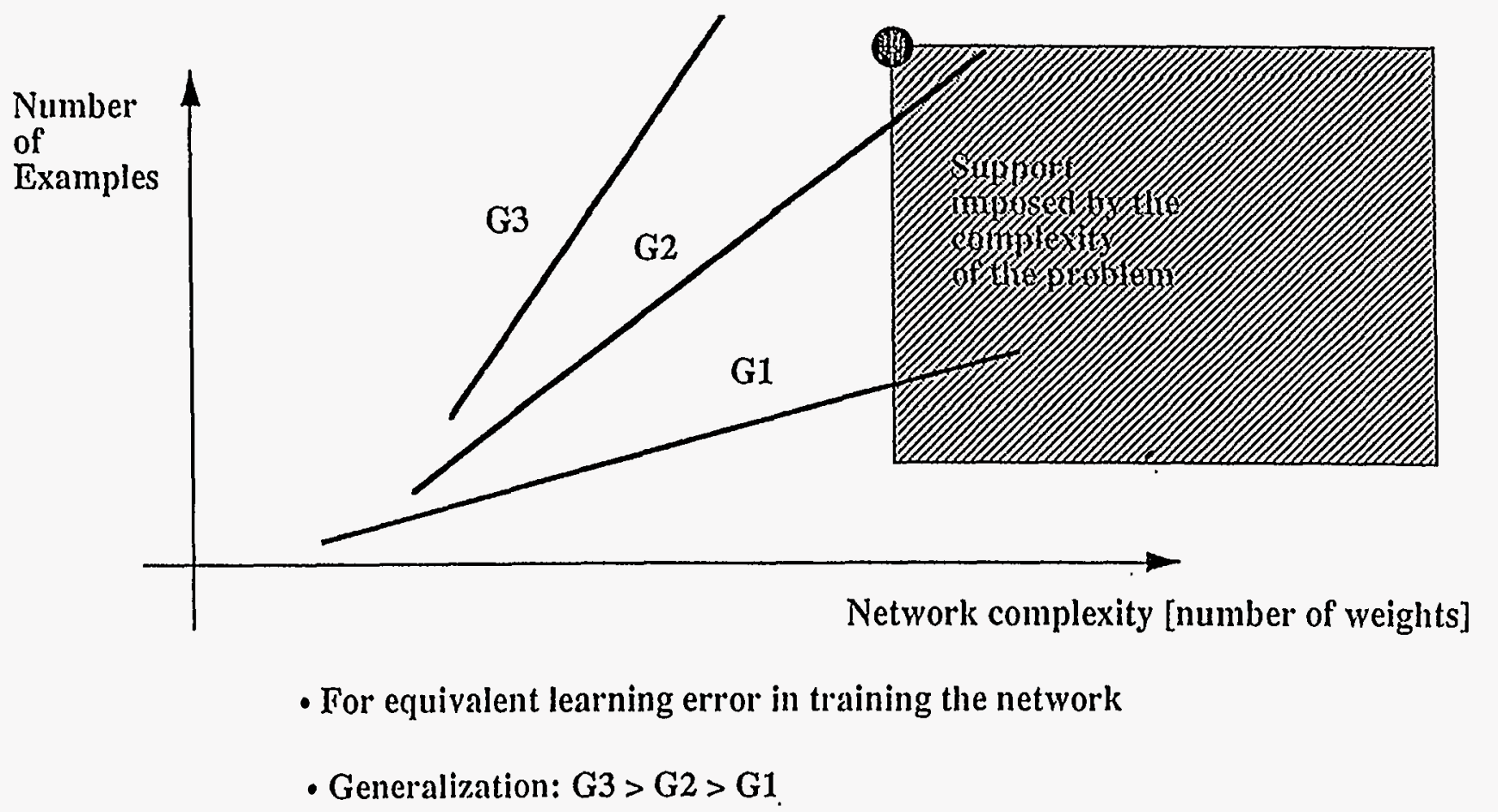

Figure 39. Plot of network complexity versus number of training examples. 
related to backpropagation networks, include the well-known interpolation technique, Radial Basis Functions method (RBF). RFB theory is used to illustrate how a regularization network can approximate a mapping between a set of vectors and an output. 


\subsection{Summary and Future Directions of ANN Groundwater Management}

\subsection{Summary}

A new optimization approach in nonlinear groundwater management that employs Artificial Neural Networks (ANNs) and the genetic algorithm (GA) is presented and demonstrated here (Section 3.0). Descriptions are included of procedures for training and testing a network for example remediations. Section 4.0 explores tools to better understand how the neural network has encoded information on the relative importance of the input parameters (primarily wells in this work) into the weights of the network. This is intended to make the ANN groundwater management more accessible and less of a "black box". Section 5.0 presents example field-scale applications from ongoing remediation optimization at Lawrence Livermore National Laboratory. Section 6.0 discusses significant issues in ANN which affect network design, training, and application.

The ANN groundwater management modeling approach can be summarized in the following steps:

(1) Conceptualization and formulation of the management problem; choice of objective functions and constraint functions.

(2) Incorporation of the management formulation into components that can be represented by a particular type of ANN network.

(3) Development of training and testing patterns.

(4) Training of the ANN; alteration of architecture to yield maximum performance of the ANN. 
(5) Search through possible pumping realizations, i.e. management solutions, with the GA to find optimal realizations. Use the trained network to predict the value of objective and/or constraint functions of the possible combinatorial pumping realizations.

(6) Using the flow and transport code to confirm that the recommended management solutions work.

This approach has several design strengths (Figure 40). The ANN management approach builds on the classical tools of hydrogeology, i.e., the numerical modeling of the flow and transport simulations. Yet, this approach also builds on the strength of two powerful modern techniques, ANN technology and GA optimization. Another design strength of the ANN approach is that the flow and transport simulations can be done in parallel. This is in contrast to conventional optimizations (using NLP) which involve sequential calls to the flow and transport code. In the ANN approach a network is trained to predict a particular outcome of a call to the flow and transport code. Due to the modular design of the ANN approach, these calls to the flow and transport code can be implemented in parallel (i.e., potentially all at the same time). Also, if the appropriate outputs are saved, these calls form a body of knowledge which can be "recycled", i.e., used to train other networks to predict output for different combinations of objective functions. After a network has been suitably trained, the GA begins a search through possible pumping pattern realizations using the trained ANN to predict the outcome and subsequently "how good" each pumping pattern realization is. The GA is a robust search procedure which uses both structure and randomness to develop new populations of vector patterns. It should also be noted that the GA is not limited to objective functions which are continuous or differentiable like the conventional nonlinear programming steepest descent search objective functions. The performance of the ANNs in predicting the output for pumping patterns not seen before was reasonably accurate for both discrete Boolean ( 1 or 0$)$ output, as well as more complex continuous output (i.e., mass output as well as multiple objectives involving a combination of remediation cost, plume containment, and clean-up time). Let us discuss some of the advantages of this approach in more detail. 


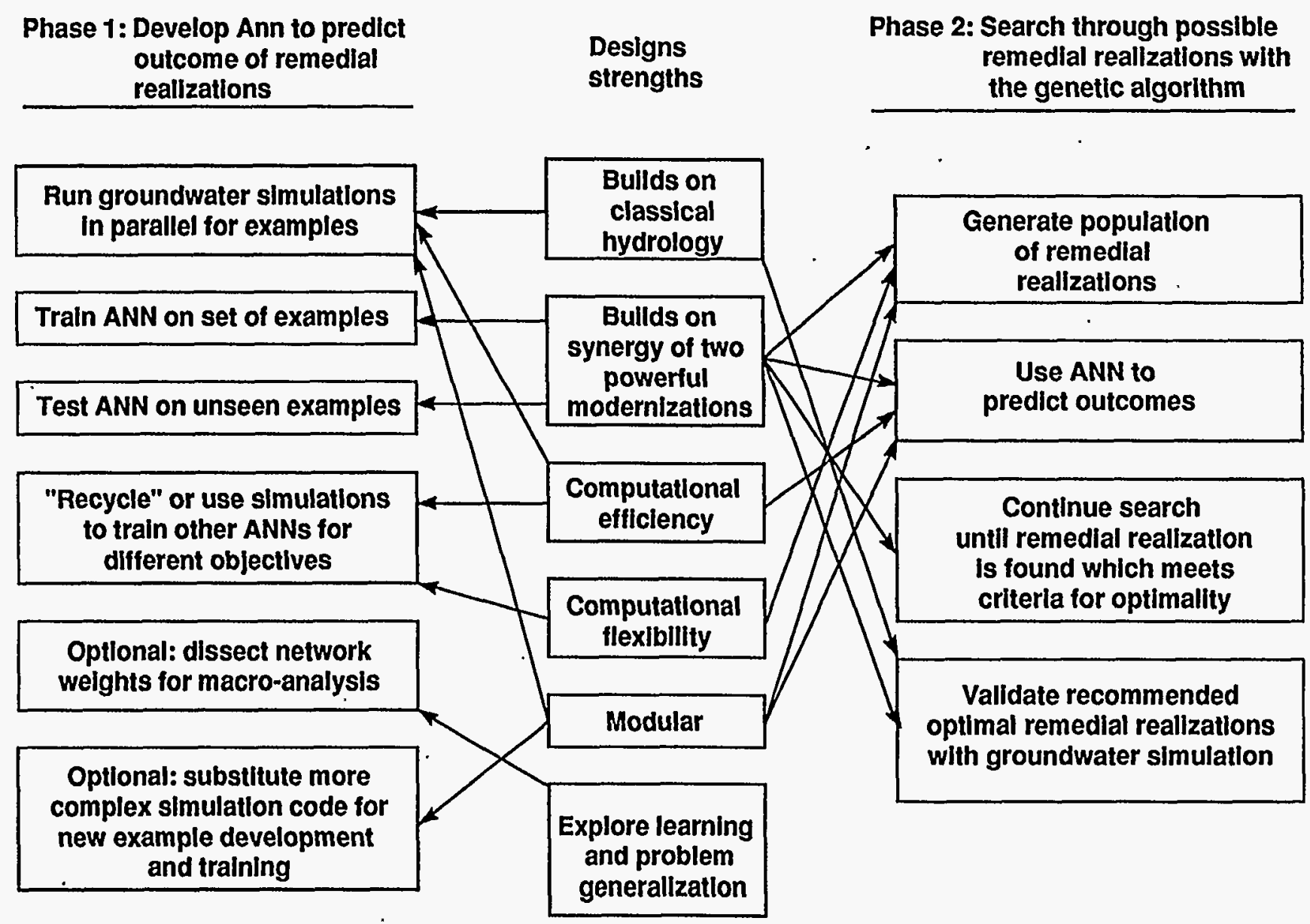

Figure 40. Design strengths of ANN groundwater management. 
(1) Less computational burden and more flexibility. In the example remediations, the ANN groundwater management solutions are consistent with the more conventional nonlinear programming groundwater management solutions with fewer calls to the flow and transport code. Calls to the flow and transport code are the main component of most groundwater management models. In addition, the potential for parallel processing of the calls to the flow and transport code and for "recycling" of the simulation should result in significant reduction of real time savings as well as computational burden. This is especially important in field-scale applications.

Let us consider an example to illustrate the strength of this approach over conventional optimization methods due to the parallel processing and "recycling" potential of the flow and transport simulations. Consider that 200 calls by a conventional method with each taking one hour of CPU can find a reasonably optimal management solution. This conventional method would have 200 real time hours in a sequential call of the flow and transport simulator necessary to determine the optimal value of the objective function. In contrast, the same CPU requirements in the ANN approach could be attained in one real-time hour if 200 machines were available. Having access to 200 machines is not unrealistic considering many facilities network their workstations these days. Moreover, this knowledge base of 200 flow and transport runs could be analyzed again and again for different objectives of remediation. Thus, one could retrain the network with different outputs (i.e., objectives) or multiple outputs (i.e., multiple objectives) without additional CPU burden of flow and transport simulations. Such a recycling of the flow and transport simulations would not be possible with a sequential optimization because the gradient of the objective function which drives the search would in most cases be different for different objective functions. Thus, for a new objective function, one might be able to use the saved outputs on the first calculation of the Jacobinan (i.e., approximately as many runs as the number of decision variables), but after that a new sequence of decision variable perturbations would be chosen based on this Jacobian. The flow and transport simulations are by far the most 
time consuming component of optimizing remediation, thus the ANN approach has considerable advantages of computational efficiency and flexibility.

2) More independence from the flow and transport code than most other optimization drivers. Typical optimization methods involve linking the flow and transport code to the optimization methodology in a fairly rigorous manner, with arrays such as pumping rates and concentrations at control locations being passed between the optimization driver and the groundwater simulation code. The nonlinear programming groundwater management model has been viewed as having the advantage of employing the flow and transport code as a somewhat independent subroutine of the management model [Gorelick et al., 1984]. In this modular approach, the flow and transport simulation submodel may be developed or expanded before linkage to the optimization model. Yet the ANN approach affords even more latitude, as the flow and transport simulation model does not need to be a subroutine of the network. This independence has subsequent advantages of increased opportunities for parallel processingras discussed above, ease of extension to more complex transport codes, and ease of addition of more objectives, as discussed below.

Extension of the methodology to optimize such remediation alternatives as injection or extraction of steam for steam displacement of contamination and injection or extraction of nutrients for a biological curtain will be easier without the necessity of passing arrays between the optimization driver and the flow and transport code. While the methodology is presented as nonlinear to incorporate solute transport directly into groundwater management, simplifications to linear applications would be straightforward, as would be applications to nonlinear problems in other areas of management concern, such as salt-water intrusion.

The independence of the ANN management approach increases the ease of incorporating multiple objectives. The addition of output components within the training and testing patterns or of postprocessing to rank the ANN-recommended management solutions is more straightforward than the conventional nonlinear programming management approach. This is particularly true where multiple technologies are involved. To give examples of this 
advantage, let us consider two objectives of minimizing cost and maximizing mass output. In the NLP management approach one would have to choose a weighting of these objectives, say making the cost objective twice as important as the mass output, in order to began a sequential search through possibilities of pumping. If one wanted to change these weights, say making them of equal value, one would have to began the sequential search again in a new series of flow and transport simulations. Or if one wanted to forget about the mass output objective, one would have to start the NLP search through the flow and transport simulations once again. In contrast, with the ANN management approach one would have only one set of flow and transport simulations to determine the relevant cost and mass output "answers" for a set of training and testing pumping realizations. The number of simulations for the ANN approach is predicted to be on the order of the number needed for one run of the NLP approach. As noted before the real-time burden of these calls can be reduced if they are run at the same time on several machines. Now, two ANN can be trained, one to predict the cost of remediation, and one to predict the mass output removed. Searches with the GA can be done again and again with different weightings of these two objective functions used as the fitness measure. The computational burden of this training and GA search is negligible compared to another run of the NLP management model.

If a different discrete technology of a slurry wall or lining of a pond is under consideration, the NLP approach cannot be used without incorporation of mixed interger programming. However, in the ANN management approach, such a technology can be considered by simply including a representation of this technology in the input vector and training runs which have this technology implemented.

The ANN groundwater management model presented does benefit from robust optimization-theory techniques as does the more conventional approach. The difference is that the nonlinear programming management model calls the flow and transport code in a sequential manner while the optimization techniques are being applied. In contrast, the ANN management approach uses optimization techniques during the training of the network (conjugate gradient 
technique) and after the network is trained (genetic algorithm) when no calls to the flow and transport code are necessary. The flow and transport code has already been called many times to develop the training set, but it does not have to be called again until validation of the recommended solutions. The separation of the flow and transport code runs from the optimization and the lack of necessity for sequential calls to the code allow for the possibility of parallel processing of the flow and transport runs.

3) Capability of making use of hydrologic insight. In many other optimization approaches, the search for improved management solutions is not impacted by or is impervious to hydrologic insight of researchers using the optimization techniques. Adequate formulation, of course, benefits from hydrologic insight, but the search itself does not. Most optimization approaches employ searches directed by variations of the gradient descent method and seek to randomize the search when possible to ensure coverage of the search space. The ANN approach can make use of these methods, but the manner in which it does still makes room for hydrologic insight. The ANN approach benefits from the robust optimization techniques of variations on the gradient descent method, in this study the conjugate gradient method, during the minimization of error in the training of the network. Also, coverage of the search space can be encouraged by randomization of training patterns. However, the hydrogeologic insight of researchers can impact the network by being used to develop and include "good" examples to train the network. Results of this study may suggest that the network performance can be increased more rapidly with such insight. This can be particularly useful where the flow and transport code is applied to a large, complex field site, and there exists some hydrologic insight on the part of researchers working with the remediation project. Thus, previous investment in expensive modeling can be put to use in the ANN groundwater management approach.

\subsection{Limitations}

There are limitations to the current state of this work, such as three-dimensionally complex alluvial deposits being modeled in an areal 2-D manner and various flow and transport 
parameters being considered to be spatially constant. This is not a limitation of the advantages of the approach, but rather a limitation of the geologic and hydrogeologic basis (i.e., simulations used in this work) upon which the ANN appraoch or any other groundwater management approach will be made. Also while the number of locations preselected for evaluation has been somewhat large relative to what has been considered with the more conventional nonlinear programming groundwater management, some field-sites may need to evaluate more than 30 possible well locations. Another limitation is that thus far the pumping well levels have been considered discrete, because pumping at full well yield appears to be good engineering practice for the relatively small well yields at many field sites, and LLNL in particular. Also the pumping is considered to be constant over the entire 40 years of simulations. These limitations are not limitations of the ANN methodology, but rather current boundaries of the work done so far. These limitations could be overcome with changes in problem formulation and an increase in the dimensionality of the input vector, requiring more training and testing examples. Several of these limitations will be approached in the near future as discussed below.

A three-dimensional model may also be used with an increase in the dimensionality of the input vector to the ANN representing a particular pumping realization. For example, instead of one input for a well location, one might have used three, representing one well location with three different rates of pumping at three different depths or three well locations with different rates of pumping at three different depths. This extension is not planned in the near future because the need for a three-dimensional model at the field-site has not been established.

\subsection{Future Extensions}

Future extensions are planned to 1) train the network with continuous values of pumping in the input variables, 2) train the network with an $x$ and $y$ component for each well, mixing many well locations into the training set to see if the network can successfully interpolate to predict outcomes for a wider range of possible well locations, 3) break the 40 years into smaller management periods between which there can be changes in the pumping levels (i.e., 
adaptive pumping, multiple pumping periods), and 4) train the network with stochastic conductivity information in order to approach the issue of certainty.

In the case of the first extension, an ANN can handle the input patterns being continuous functions instead of boolean ( 1 or 0 ). Indeed, additional training and testing patterns may not be absolutely necessary in the first example, because an ANN is a very powerful interpolator and would make an interpolation if trained on the endpoints, $(1$ or 0$)$ and then feed some intermediate input value (e.g., 5 representing well on at half of the potential well yield). There will be some necessary alterations of the GA in this case in order to encode more continutity in the pumping levels.

The need for additional training and testing patterns is predicted in the second three extension cases due to an increase the dimensionality of the input vector. An increase in the input vector would increase the network weights, assuming a constant number of hidden nodes. Note with an increase in dimensionality the number of hidden units may need to be increase. The interpolation for variations in $x$ and $y$ values is also possible with the current ANN methodology. The multiple pumping periods can be handled by multiplying the number of input parameters by the the number of pumping periods. For example to have 20 wells and three pumping periods one could have 60 input parameters with the first 20 correlating to pumping of the 20 wells in the first pumping periods, the second 20 correlating to pumping of the 20 wells in the second pumping period and so on. To address the stochastic problem one might consider incorporating the realizations of conductivity into the input vector as well. This was the approach of Ranjithan (1991) who encoded 100 hydraulic conductivity values into the input vector of an ANN. Ranjithan's network still relied on the linear programming's sequential calls to the flow code, and could become fairly cumbersome for the nonlinear high dimensionality problems being considered here at the field-scale. However, one might approach the problem with a series of networks in some combination of the approach of this work and Ranjithan's work to address uncertainty without the sequential calls to the flow and transport code. For example, one might generate various hydraulic conductivity realizations and use the 
ANN management approach to determine and optimal management solution. One might then train another ANN upon the hydraulic conductivity realizations and optimal management solutions, as Ranjithan did, to then search through many hydraulic conductivity realizations to determine those most likely to be the most difficult to remediate.

The development of ANN groundwater management methodology is in its infancy. Despite its youth, the results of this work suggest that ANN groundwater mangement can be a robust and flexible tool to aid decision-making in remediation. The noted advantages of the ANN approach should be significant and promising for field-scale applications. 


\section{References}

Aguado, E., and I. Remson, Groundwater hydraulics in aquifer management, J. Hydraul. Div. Am. Soc. Civ. Eng., 100(HY1):103-118, 1974.

Ahlfeld, D., Two-stage groundwater remediation design, Journal of Water Resources Planning and Management, 116( 4), 517-529, 1990.

Ahlfeld, D., Designing contaminated groundwater remediation systems using numerical simulation and nonlinear optimization, Ph.D. dissertation, Princeton, 1987.

Ahlfeld, D.P., J.M. Mulvey, and G.F. Pinder, Contaminated groundwater remediation design using simulation, optimization, and sensitivity theory number 1: Model development, Water Resour. Res. 24(3):431-441, 1988a.

Ahlfeld, D.P., J.M. Mulvey, and G.F. Pinder, Contaminated groundwater remediation design using simulation, optimization, and sensitivity theory number 2: Analysis of a field site, Water Resour. Res., 24(3):442-452, 1988b.

Ahlfeld, D.P., J.M. Mulvey, and G.F. Pinder, Designing optimal strategies for contaminated groundwater remediation. Advances in Water Resources,9(2):77-84, 1986.

Alley, W., Regression approximations for transport model constraint sets in combined simulation-optimization studies, Water Resour. Res., 22, 581-586, 1986.

Anderson, T., O.R. Burt, and D.T. Fractor, Privatizing groundwater basins: a model and its application: in Water Rights, Scarce Resource Allocation, Bureaucracy, and the Environment, by T.L. Anderson (ed), Pacific Institute for Policy Research, San Francisco, 348 p., 1983.

Ash, T.,Dynamic node creation in backpropagation networks, University of California at San Diego, Institute for Cognitive Science Technical Report 8901, 1988.

Atwood, D.F., and S.M. Gorelick, Hydraulic gradient control for groundwater contaminant removal, Journal of Hydrology, 76:85-106, 1985.

Atwood, D.F., Management of contaminated groundwater with aquifer simulation and linear programming: the development of a hydraulic gradient control procedure, M.S. thesis, Dept. of Applied Earth Sciences, Stanford University, Stanford, CA., 1984.

Bahr, J.M., Analysis of nonequilibrium desorption of volatile organics during field test of aquifer decontamination, I. Contam. Hydrol., 4:201-222, 1989.

Bear, J., Hydraulics of Groundwater, McGraw-Hill, New York, N.Y., 1979. 
Bear, J., Dynamics of Fluids in Porous Media, American Elsevier, New York, NY, 1972

Becker, S. and Y. Le Cun, Improving the Convergence of Back-Propagation Learning with Second Order Methods, Proc. of the 1988 Connectionist Models Summer School, Morgan Kaufman, pp. 29-37, 1989.

Bredehoeft, J.D., and R.A. Young, Conjunctive use of groundwater and surface water for irrigated agriculture: risk aversion, Water Resour. Res., 19(5):1111-1121, 1983.

Bredehoeft, J.D., and R.A. Young, The temporal allocation of groundwater: A simulation approach, Water Resour. Res., 6:3-31, 1970.

Baum, E., Generalizing backpropagation to computation, In J. Denker (Ed.), AIP Conference Proceedings 151: Neural Networks for Computing, New York, American Institue of Physics, pp. 47-52, 1986.

Canales, T.W., V.M. Johnson, J.P. Ziagos, A.F.B. Tompson, M.P. Maley, P.F. McKereghan, E.M. Nichols, PLANET an interactive computer system for the rapid generation and evaluation of ground water pump and treat scenarios, Proceedings of Spectrum ' 92 Nuclear and Hazardous Waste Management International Topical Meeting, Bosie, Idaho, August 23-27, 1992.

Caudill, M. and C. Butler, Naturally Intelligent Systems, MIT Press, Cambridge, MA, 1990.

Chaudry, M.T. J.W. Labadie, W.A. Hall, and M.L. Albertson, Optimal conjunctive use model for the Indus basin, Journal of Hydraulics Division, American Society of Civil Engineering, . 100:667-698, 1974.

Cheesman P.C., On finding the most probable model, in J. Shrager and P. Langley (eds.) Computational Models of Scientific Discovery and Theory Formation, Morgan Kaufmann, 1990.

Clement, W. W., R. M. Inigo, and W. S. McVey, Synaptic strengths for neural simulation of the Traveling Salesman Problem, Proc. Applications of Artificial Intelligence SPIE, 927:373-380, 1988.

Dayhoff, J., Neural Net Architectures, Van Nostrand Reinhold, New York, NY, 1989.

Deninger, R.A., Systems analysis of water supply systems. Water Resour. Bull., 6(4):573-579, 1970.

Denker, J., The power of generalization in a back-propagation network: A case study, Workshop on Neural Network Devices and Application, Jet Propulsion Laboratory, pp.202-213, 1987.

Dogru, A.H., and J.H. Seinfeld, Comparison of sensitivity coefficient calculation methods in automatic history matching. Society of Petroleum Engineering Journal, 21: 551-557, 1981.

Dougherty, D.E., and R.A. Marryott, Optimal groundwater management, 1. Simulated annealing, Water Resour. Res., 27,(10):2493-2508, 1991. 
Dowla, F.U., and S. R. Taylor, and R.W. Anderson, Seismic Discrimination with Artificial Neural Networks: Preliminary Results with Regional Spectral Data, Bulletin of the Seismological Society of America, 80(5):1346-1373, October 1990.

Dowla, F. U., E. B. Talbot, and V. Vemuri, Artificial neural networks: an application for the classification of seismic signals, Technical Report no. UCID-21552, Lawrence Livermore National Laboratory, Livermore CA, 1988.

Dresen, M.D., E.M. Nichols, W.A. McConachie, K.S. Buchanan, and W.F. Isherwood, Remedial alternatives for VOCs in ground water west of LLNL, Lawrence Livermore National Laboratory, Livermore, CA,UCAR-10202, 1987.

Dresen, M.D., and F. Hoffman, Volatile organic compounds in ground water west of LLNL, Lawrence Livermore National Laboratory, Livermore, CA, UCRL-53740,70p, 1986.

Gailey, R.M., A.S. Crowe, and S.M. Gorelick, Coupled process parameter estimation and prediction uncertainty using hydraulic head and concentration data, Advances in Water Resources: Parameter Identification in Groundwater Flow, Transport and Related Processes: 2-Advanced Applications, 1991.

Gallinari, P., Thiria, S., and F. Fogelman Soulie, Multilayer perceptrons and data analysis,

Proceedings of the IEEE International Conference on Neural Networks: San Diego: IEEE, Vol. I:391-399, 1987.

Gill, P. D., W. Murray, M. A. Saunders, and M. H. Wright, Users guide for NPSOL (version 4.0): A FORTRAN package for nonlinear programming, Tech. Rep. SOL 86-2, Syst. Optimiz. Lab., Stanford University, Stanford, CA., 1986

Gill, P. E., W. Murray, and M. H. Wright, Practical Optimization, Academic Press, NY, 1981. Goldberg, D.E., Genetic Algorithms in search, optimization, and machine learning, AddisonWesley: Reading, MA, 1989.

Gorelick, S.M., C.I. Voss, P.E. Gill, W. Murray, M.A. Saunders, and M.H. Wright, Aquifer reclamation design: the use of contaminant transport simulation combined with nonlinear programming, Water Resour. Res., 20(4):415-427, 1984.

Gorelick, S.M., A review of distributed parameter groundwater management modeling methods, Water Resour. Res., 19(2):305-319, 1983.

Gorelick, S. M., and I. Remson, "Optimal location and management of waste disposal facilities affecting groundwater quality," Water Resour. Bulletin, 18(1):43-51, 1982.

Grossberg, S., (ed.), Neural Networks and Natural Intelligence, MIT Press, Cambridge, MA, 1988.

Grossberg, S., Adaptive pattern classification and universal recoding: II. Feedback, oscillation, olfaction, and illusions, Biological Cybernetics, 21:145-159, 1976.

Grossberg, S., Classical and instrumental learning by neural networks., Progress in Theoretical Biology, (3):51-141, 1974 
Hebb, D., The Organization of Behavior, Wiley, New York, NY, 1949.

Hecht-Nielsen, R., Neurocomputing, Addison-Wesley, Reading, MA., 1989.

Hecht-Nielson, R., Neurocomputing: picking the human brain, IEEE Spectrum, March, pp.36-41, 1988.

Hegde, S.U., J.L. Sweet, and W.B. Levy, Determination of parameters in a Hopfield/Tank computational network, Proc. International conf. on Neural Networks I:291-298, July 1988.

Holland, J., Adaption in natural and artificial systems , MIT Press: Cambridge, MA, 1992.

Hopfield, J., Neurons with graded response have collective computational properties like those of two-state neurons, Biophysics, Proc. Natl. Acad. Sci., USA, 81:3088-3092, 1984.

Hopfield, J.J. and D.W. Tank, Neural computation of decisions in optimization problems, Bio. Cybern, 52:141-152, 1985.

Hopfield, J.J., Neural networks and physical systems with emergent collective computational properties, Proc. Natl. Acad. Sci. (79):2554-2558, 1982.

Hunt, J.R., N. S. Sitar, and K.S. Udell, Nonaqueous phase liquid transport and cleanup, 1, Analysis of mechanisms and 2, Experimental studies, Water Resour. Res., 24(8):247-1270, 1988.

Irie, B. and S. Miyake, Capabilities of three-layered perceptrons, Porceeding of the IEEE International Conference on Neural Networks: I:641-648, 1988.

Jang, G, F. Dowla, and V. Vemuri, Application of Neural Networks for Seismic Phase Identification, in Pro. of IEEE Joint Conference on Neural Networks (Singapore), December 1991.

Jeffrey, W., and R. Rosner, Optimization Algorithms: Simulated Annealing and Neural Network Processing, The Astrophysical Journal, 310:473-481,1986.

Johansson, E.M., F.U. Dowla, and D.M. Goodman, Backpropagation Learning for Multi-Layer Feed-Forward Neural Networks Using the Conjugate Gradient Method, International Journal of Neural Systems, Vol. 2:291-301, 1992. Also, Lawrence Livermore National Laboratory UCRL-JC-104850, 1990.

Kirkpatrick, S., C.D. Gelatt, and M.P. Vecchi, Optimization by simulated annealing, Science, 220(4598):671-680, 1983.

Klopf, A.H., Drive-Reinforcement Learning: A Real-Time Learning Mechanism for Unsupervised Learning, IEEE First International Conference on Neural Networks, II:441-446, 1987.

Kolmogorov, A.N., On the representations of continuous functions of many vaiables by superpositions of continuous functions of one variable and addition. Dokl. Akad. Nauk USSR, 114, (5):953-956, 1957. 
Kramer, A.H. and A.S. Vincentelli, Efficient Parallel Learning Algorithms for Neural Networks, in D.S. Touretzky (Ed.), Advances in Neural Information Processing Systems I, Morgan Kaufman, pp.40-48, 1989.

Kuhn, H.W., and A.W. Tucker, Nonlinear programming. In J. Neyman (Editor), Proceedings of the Second Berkeley Symposium on Mathematical Statistics and Probability, Berkeley. University of California Press, pp.481-492, 1951.

Le Cun, Y., Une procedure d'apprentissage pour reseau a seuil assymetrique (A learning procedure for asymmetric threshold network), Proceedings of Cognitiva 85, pp. 599-604, June 1985.

Lefkoff, L. J., "The hydrologic and economic effects of water marketing on an irrigated streamaquifer system subject to salinity degradation," unpublished Ph.D. dissertation, Stanford University, Stanford, CA, 1988.

Lefkoff, L.J., and S. Gorelick, "Design and cost analysis of rapid aquifer restoration systems using flow simulation and quadratic programming," Groundwater, 24 (6):777-790, 1986.

Lefkoff, L.J. and S. Gorelick, Groundwater contamination and reclamation, American Water Resources Association, pp. 125-131, August 1985.

Lippman, R.P., Introduction to computing with neural nets, IEEE ASSP Magazine 4:4-22, 1987.

Luenberger, D.G., Linear and Nonlinear Programming.Addison-Wesly, Inc.: Reading, MA, 491pp, 1984.

Maren, A.J., A Logical Topology of Neural Networks, in Pro. of the Second Workshop on Neural Networks: Academic/Industrial/ NASA/Defense (WNN-AIND 91), February 1991.

Maren, A.J., C.T. Harston, and R.M. Pap, Handbook of Neural Computing Applications: New York: Academic, 1990.

McClelland, J.L., Rumelhart, D.E. and the PDP Research Group, Parallel Distributed Processing: Explorations in the Microstructure of Cognition, Vol. 2, MIT Press: Cambridge, MA, 1986.

McCormick, G.P., Nonlinear Programming. John Wiley: New York, NY, 1983.

McCulloch, W.S. and W. Pitts, A logical calculus of the ideas imminent in nervous activity, Bulletin of Mathematical Biophysics, 5:115-133, 1943.

Minsky, M. and S. Papert, Perceptrons, Expanded Edition, MTT Press: Cambridge, MA, 1988.

Minsky, M. and S. Papert, Perceptrons, MIT Press: Cambridge, MA, 1969.

Moore, B. and T. Poggio, ART1 and pattern clustering algorithms, Neural Networks Supplement: INNS Abstracts, 1,116, 1988.

Murphy, J. H., Tutorial: Neural network learning algorithms, in Pro. of the Second Workshop on Neural Networks Academic/ Industrial/ NASA/ Defense (WNN-AIND 91), February 1991. 
Murtagh, B.A. and M.A. Saunders, "MINOS/AUGMENTED User's Manual," systems Optimization Laboratory, Technical Report 80-14, Department of Operations Research, Stanford University, Stanford, CA, 51p, 1980.

Musari, M., A. Rajavelu, S. Sahai, and J. Zhao, Analysis and generalization of back propagation in neural networks, Neural Networks Supplement: INNS Abstracts, I, 118, 1988.

Parker, D., Optimal algorithms for adaptive networks: Second order back propagation, second order direct propagation, and second order Hebbian learning, Proceedings of the IEEEE First International Conference on Neural Networks : San Diego, Vol, III:715-720, 1987.

Parker, D.B., Learning-logic, Technical Report TR-47, MIT Center for Computational Research in Economics and Management Science: Cambridge, MA, 1985.

Poggio, T. and F. Girosi, Networks for Approximation and Learning, Proceedings of the IEEE, 78(9):1481-1497, 1990.

Polak, E. and G. Ribiere, "Note sur la Convergence de Methods de Directions Conjures," Revue Francaise Information Recherche Operationnelle, Vol. 16:35-43, 1969.

Powell, M.J.D., Introduction to constrained optimization. In P. E. Gill and W. Murray (Editors), Numerical Methods for Constrained Optimization. Academic Press: London and New York, pp.1-28, 1974.

Powell, M.J.D., On the convergence of the variable metric algorithm. J. Inst. Maths. Applics, 7:2136, 1971.

Press, W.H. , Numerical Recipes: the Art of Scientific Computing, Cambridge University Press: Cambridge, 1986.

Psaltis, D. and M. Neifield, The emergence of generalizaiton in networkds with constrained representation, Proceedings of the IEEE International Conference on Neural Networks: San Diego: IEEE, Vol.. 1:371-381, 1988.

Ranjithan, S., J.W. Eheart, and J.H. Garrett, Jr., Application of neural network in groundwater remediation under conditions of uncertainty, in Proceedings of the International Workshop on New Uncertainty Concepts in Hydrology and Water Resources, Madralin, Poland, September 24-26, 1990.

Reichard, E., Hydrologic influences on the potential benefits of basinwide groundwater management, Water Resour. Res., 23(1):77-91, 1987.

Remson, I., and S.M. Gorelick, Management models incorporating groundwater variables, Proceedings of Operations Research in Agriculture and Water Resources, Jerusalem, 25-29 Nov: D. Yaron and C. Tapiero (Eds.), North-Holland Pub. Co., pp. 333-356, 1980.

Rissanen, J., Stochastic Complexity in Statistical Inquiry, World Scientific, 1989.

Rogers, L.L., History Matching to Determine the Retardation of PCE Transport, Ground Water, 30 (1):50-60, January-February 1992. 
Rogers, L.L., Nonlinear simulation-management methodology involving solute transport with multiple management periods. Lawrence Livermore National Laboratory, Livermore, CA,UCRL-102152, 1991.

Rogers, L.L., Nonlinear optimization remediation of contaminated aquifers incorporating linear sorption: Principles and case history, AIH Hydrological Science and Technology, Vol 5:157169.

Rosenblatt, F., Principles of Neuro-dynamics: Perceptrons and the Theory of Brain, Mechanisms, Spartan Books, Washington, D.C., 1962.

Rosenblatt, F., The perceptron: A probabilistic model for information storage and organization in the brain, Psychological Review, 65:386-408, 1958.

Rumelhart, D.E., J.L. McClelland, and the PDP Research Group, Parallel Distributed Processing: Explorations in the Microstructure of Cognition, vol. 1, MTT Press: Boston, 1986.

Rumelhart, D.E., personal communication, Department of Psychology, Stanford University, Stanford, CA.

Rutenbar, R.A., Simulated annealing algorithms: an overview, IEEE Circuits and Devices Magazine, pP.19-26, January 1989.

Sietsma, J. and R. Dow, Neural net pruning-why and how, Proceeding of the IEEE International Conference on Neural Networks: San Diego, Vol., I:325-333, 1988.

Simpson, P.K., Artificial Neural Systems: Foundations, Paradigms, Applications, and Implementations, New York: Pergamon, 1990.

Smieja, F., The significance of underlying correlations in the training of a layered net, Neural Abstracts, 1:468, 1988.

Szu, H., Fast TSP algorithm based on binary neuron output and analog neuron input using the zero-diagonal interconnect matrix and necessary and sufficient constraints of the permutation matrix, Proc. International Conf. on Neural Networks II:259-266, July 1988.

Sutton, R., Two problems with backpropagation and other steepest-descent learning procedures for networks, Proceedings of the Eighth Annual Conference of the Cognitive Science Society, Hillsdale, NJ: Lawrence Erlbaum Associates, pp 823-831, 1986.

Tank, D. W., and J.J. Hopfield, Simple neural optimization networks: an A/D converter, signal decision circuit, and a linear programming circuit, IEEE Trans. on Circuits and Systems, Vol., CAS-33, 5:553-541, May 1986.

Thorpe, R.K., W.F. Isherwood, M.D. Dresen, and C.P. Webster-Scholten (Editors), Draft CERCLA remedial investigations report for the LLNL/Livermore Site, Lawrence Livermore National Laboratory, Livermore, CA.,UCAR-10299DRAFT, 1989. 
Tishby, N., E. Leven, and S.A. Solla, Consistent inference of probabilities in layered networks:

Predictions and generalizations, Proc. First Int'1. Joint Conf. Neural Networks, Washington, D.C., June 18-22, 1989, П1:403-409, 1989.

Tompson, A.F.B., 1990. Flow and transport within the saturated zone beneath Lawrence Livermore National Laboratory: modeling considerations for heterogeneous media. Lawrence Livermore National Laboratory, Livermore, CA.UCID-21828, 1990.

Voss, C.I. , A finite-element simulation model for saturated-unsaturated, fluid-densitydependant groundwater flow with energy transport or chemically-reactive single-species solute transport, U.S. Geological Survey, Water Resources Investigations Report, 84-4369, 409p, 1984.

Wagner, B. J., "Optimal Groundwater Quality Management Under Uncertainty," unpublished Ph.D. dissertation, Stanford University, Stanford, CA, 1988.

Wagner, B.J., and S.M. Gorelick, Reliable aquifer remediation in the presence of spatial variable hydraulic conductivity: From data to design, Water Resour. Res., 25(10):2211-2225, 1989.

Wagner, B.J., and S.M. Gorelick, Optimal groundwater quality management under parameter uncertainty, Water Resour. Res., 23(7):1162-1174, 1987.

Wagner, B.J., and S.M. Gorelick, A statistical methodology for estimating transport parameters: theory and applications to one-dimensional advective-dispersive systems. Water Resour. Res.,22(8):1303-1315, 1986.

Walbridge, S." "Development of Optimization Techniques and Application to Purge Well Network Design for the Gloucester Landfill," M.S. thesis, Dept. of Applied Earth Sciences, Stanford University, Stanford, CA, 1984.

Wanakule, N., L.W. Mays, and L.S. Lasdon, Optimal management of large-scale aquifers: Methodology and applications, Water Resour. Res., 22(4):447-465, 1986.

Wasserman, P.D., Neural Computing: Theory and Practice, Van Nostrand Reinhold: New York, 1989.

Weigend, A.S. and D.E. Rumelhart, and B.A. Huberman, Generalization by weight-elimination with application to forecasting, Advances in Neural Information Processing 3 (NIPS*90), edited by R.P. Lippmann, J. Moody and D.S. Touretzky, San Mateo, CA, Morgan Kaufmann, 1991 .

Weigend, A.S., B.A. Huberman, and D.E. Rumelhart, Predicting sunspots and currency rates with connectionist networks, in M. Casdagli and S. Eubank (eds.), Proc of the 1990 NATO Workshop on Nonlinear Modeling and Forecasting.(Santa Fe), Addison-Wesley, 1991b. 
Weigend, A.S. and D.E. Rumelhart, and B.A. Huberman, Back-propagation, weight-elimination and time series prediction, In D.S. Touretzky, J.L. Elman, T.J. Sejnowski, and G.E. Hinton (editors), Proceedings of the 1990 Connectionist Models Summer School, (105), Morgan Kaufmann, 1990.

Werbos, P., Beyond Regression: New Tools for Prediction and Analysis in Behavioral Sciences, PhD. Thesis, Harvard University, Cambridge, MA, 1974.

White, H., Some asymptotic results for learning in single hidden layer feedforward network models, University of California at San Diego, Department of Economics, Discussion Paper 87-13, 1988.

Widrow, B. and M.E. Hoff, Adaptive switching circuits, IRE WESCON Convention Record, pp. 96-104, 1960.

Widrow, B. and S.D. Stearns, Adaptive Signal Processing, Signal Processing Series, Prentice-Hall, Englewood Cliffs, NJ, 1985.

Willis, R., Optimal groundwater quality management: Well injection of waste water, Water Resour. Res., 12(1):47-53, 1976.

Willis, R., and B.A. Finney, Optimal control of nonlinear groundwater hydraulics: Theoretical development and numerical experiments, Water Resour. Res., 21(10):476-1482, 1985.

Young, R.A., and J.D. Bredehoeft , Digital computer simulation for solving management problems of conjunctive groundwater and surface water systems, Water Resour. Res., 8:533$556,1972$.

Yves, C, Generalization performance of overtrained networks, in L.B. Almeida and C.J. Wellekens, editors, Neural Networks. Proc. of 1990 EURASIP Workshop, Vol. 412:46, of Lecture Notes in Computer Science, Springer, 1990. 


\section{Appendix A \\ Conjugate Gradient Backpropagation Learning Algorithm}

The backpropagation leaming algorithm that we are using in this study employs the conjugate gradient method to improve learning rates. This method is outlined in some detail by Johansson et al. [1990], where the reader is referred for a tutorial introduction to the conjugate gradient optimization method. This appendix gives a brief description of conjugate gradient backpropagation.

To apply the conjugate gradient method to backpropagation, we first view backpropagation learning as an unconstrained nonlinear optimization problem. Thus, we use a vector of independent variables, $\boldsymbol{x}$, and an objective function, $f$, defined on the vector space of $\boldsymbol{x}$, which will be minimized. We need to evaluate $f(x)$ and its gradient $g(x)=\operatorname{del} f(x)$ at any point $x$. The goal of training the network is to modify the weights in such a manner that the network outputs for each pattern match the desired outputs. Therefore, the weights will be the vector of independent variables.

Mathematically we can define $f(x)$ as

$f(x)=\frac{1}{P} \sum_{p} E_{p}$

where $P$ is the number of patterns in the training set, and $E_{p}$ is the output error for each pattern p. $E_{p}$ is defined as

$E_{p}=\frac{1}{2} \sum_{j}\left[T_{p j}-Q_{p j}(x)\right\}^{2}$ 
where $O_{p j}(x)$ and $T_{p j}$ are the predicted and desired outputs of the $j$ th output neuron for the $p$ th pattern, respectively. We have shown $O_{p j}$ as a function of $x$ to emphasize the dependency of the network outputs on the weight vector $\mathbf{x}$. Thus, the objective function is given by

$f(x)=\frac{1}{2 P} \sum_{p} \sum_{j}\left[T_{p j}-O_{p j}(x)^{2}\right]$

[A3]

One function evaluation involves passing the entire training set through the network, calculating the errors for each training pattern, and summing and normalizing the results. The cost of computing $f(x)$ increases as the size of the weight vector and the number of training patterns increase.

Computation of the gradient, $\operatorname{del} f(x)$, involves differentiation of Eq A1 with respect to $x$, resulting in

$$
g(x)=\frac{1}{p} \sum_{p} \operatorname{del} E_{p}(x)
$$

The derivation of del $E_{p}(x)$ is nearly identical to the derivation of $\frac{\partial E_{p}}{\partial w_{j i}}$ in Rumelhart et al. [1986], and is only briefly summarized here. The vector, del $E_{p}(x)$, is composed of elements $\frac{\partial E_{p}}{\partial x_{j i}}$, where $x_{j i}$ is the weight element from the $i$ th to the $j$ th neuron. The deriviative may be expressed as the product of two parts:

$$
\frac{\partial E_{p}}{\partial \mathbf{x}_{j i}}=\frac{\partial E_{p}}{\partial n e t_{p j}} \frac{\partial n e t_{p j}}{\partial \mathbf{x}_{j i}}
$$


where $\frac{\partial E_{p}}{\partial \text { net }_{p j}}$ represents the change in the input of the $j$ th neuron, and $\frac{\partial n e t_{p j}}{\partial x_{j i}}$ represents the change in the input of the $j$ th neuron due to a change in the weight. The input to the $j$ th neuron, net $_{p j}$, is defined as

$\operatorname{net}_{p j}=\sum_{i} x_{j i} O_{p i}(\mathbf{x})$

where $O_{p i}(x)$ is the output of the $i$ th neuron $\left(O_{p i}(x)\right.$ is the $i$ th input when $i$ is an input neuron). Eq. A5 can be used to show

$\frac{\partial_{n e t}}{\partial x_{j i}}=O_{p i}(\mathbf{x})$

Now we define

$\delta_{p j}=\frac{\partial E_{p}}{\partial n e t_{p j}}$

This expression differs from the $\delta_{p j}$ of Rumelhart et al. [1986] by a minus sign. Thus,

$\frac{\partial E_{p}}{\partial x_{j i}}=\delta_{p j} O_{p i}(x)$

This definition of $\delta_{p j}$ can be used, following the same arguments as Rummelhart et al. (1986), to obtain

$\delta_{p j}=-\left(T_{p j}-O_{p j}(x)\right) s_{j}^{\prime}\left(\right.$ net $\left._{p j}\right)$ 
for output neurons, where $s_{j}$ (net $_{p j}$ ) is the derivative of the semilinear activation or squashing function, and

$\delta_{p j}=s_{j}^{\prime}$ net $\left._{p j}\right) \sum_{k} \delta_{p k} x_{k j}$

for hidden-layer neurons.

Evaluating the gradient requires forward propagation of each pattern in the training set through the network to generate the neuron outputs, and then propagating the values of $\delta_{p j}$ backwards through the network. The final gradient is computed by summing del $E_{p}(x)$ over all the training patterns. The conjugate gradient method requires evaluating both the function and gradient values, and these are most efficiently calculated at the same time. 


\section{Appendix B}

\section{Nonlinear Programming Method of Groundwater Management}

The more conventional groundwater management methodology used to benchmark the ANN groundwater management methodology is described in more detail here. Two primary components of the management model are the nonlinear optimization code (NPSOL) [Gill et al. 1986] and the groundwater simulation code (SUTRA) [Voss, 1984]. The optimization code handles the input of objective functions, water quality constraints, and hydraulic constraints. The groundwater simulation code, linked as an independent submodel, controls the input of hydrogeologic data, the domain of interest, flow velocities, and transport mechanisms. The management model initializes values for the pumping rates in remediation wells. The optimization code then calls the simulation submodel to calculate the flow regime and distribution of the contaminants caused by the initial pumping rates. The optimization code will vary these pumping rates by a small increment and recall the simulation submodel to see the resulting change in the distribution. After several calls to the submodel, the optimization code will determine how the concentration change differed with respect to the changes in pumping rates of the wells. This is the numerical evaluation of the Jacobian matrix by perturbations in the pumping rates.

The optimization code can decide from the Jacobian matrix whether or not to increase or decrease the pumping level in a particular well. The entire process will continue until the optimization code's conditions for optimality and convergence are reached, or until the code 
decides that improvement in the solution cannot be made. In this modular approach, the simulation submodel may be developed or expanded before it is linked to the optimization code.

The optimization code is capable of incorporating the nonlinearity of the original constraints into the objective function of a linearly constrained subproblem. Generally, the method of the optimization code can be summarized as (1) a point is determined that satisfies the upper and lower bounds and the linear constraints and (2) iterations continue that involve (a) the solution of a quadratic programming subproblem, (b) a search direction determined with the aid of an augmented Lagrangian merit function, and (c) a quasi-Newton update of the approximate Hessian of the Lagrangian merit function. Only a brief outline of the method follows, the reader is referred to the users manual for more detail (Gill et al., 1986).

The optimization code (NPSOL) is a group of Fortran 77 subroutines that has been widely used to solve nonlinearly constrained problems. It is robust, efficient, and well suited for dealing with large, dense matrices. A smooth nonlinear function subject to constraints can be . defined as

$$
\begin{array}{ll}
\text { minimize } & F(x) \\
\text { Subject to } & l \leq\left\{\begin{array}{c}
x \\
A_{L}^{x} \\
C(x)
\end{array} \mid \leq u\right.
\end{array}
$$

where $F(x)$ (the objective function) is a nonlinear function, $A L$ is an $m_{L}$ by $n$ constant matrix of general constraints, and $C(x)$ is an $\mathrm{m}_{\mathrm{N}}$-vector of nonlinear constraint functions. The objective function, $F(x)$, and the constraint functions are assumed to be smooth, i.e., at least twicecontinuously differentiable. The variables $l$ and $u$, the upper and lower bounds, are specified for all variables and constraints to allow full generality in describing the constraints. In this study, $C(x)$ is the distribution of contaminant concentrations that is defined by the groundwater transport submodel. A viable method to solve (B1) and (B2) would be a projected Lagrangian method, which hinges on the fact that the optimal solution of (B1) and (B2) is a minimum of the 
Lagrangian function in the subspace defined by linearizations of the binding constraints. See Gill et al. [1981] for further details. Linearizations of the nonlinear constraints and the approximations of the Lagrange multipliers may be used to simplify the problems into one with only linear constraints.

A typical iteration of the method can be described as follows. Given $u_{K}$ the current best approximation to the solution; and $l_{K}$ an approximation of the Lagrange multiplier vector at the solution of (A1), the next iterate $u_{k+1}$ is the solution of the following linearly constrained subproblem

minimize $\quad f^{o}(u)-\lambda_{k} t(f-\tilde{f})+\frac{1}{2} \rho(f-\tilde{f})^{t}(f-\tilde{f})$

subject to $\quad J_{k} u \geq-f_{k}+J_{k} u_{k}$

where $f_{k}$ is $f\left(u_{k}\right) ; J_{k}$ is the Jacobian of the $f(u)$ evaluated at $u_{k} ; \rho_{\text {is a nonnegative scalar penalty }}$ parameter, and

$$
\tilde{f}(u)=f_{k}+J_{k}\left(u-u_{k}\right)
$$

(B3) - (B5) contain only linear constraints. The nonlinearities of the original problem are now contained in the objective function of the subproblem. The objective becomes complex as it consists of two components, one of which is the approximation of the Lagrangian function for the current iterate; and the other is a modified quadratic-penalty function that influences the departure of the norlinear constraints from their current linearization. Thus, the nonlinearly constrained problem of (B1) and (B2) can be solved with linearly constrained subproblems. 